\title{
Effect of Intradialytic Aerobic Exercise among Hemodialysis Patients in the United Arab Emirates on Clinical Outcomes, Barriers to Physical Activity, and Quality of Life
}

Citation for published version (APA):

Salhab, N. (2019). Effect of Intradialytic Aerobic Exercise among Hemodialysis Patients in the United Arab Emirates on Clinical Outcomes, Barriers to Physical Activity, and Quality of Life. [Doctoral Thesis, Maastricht University]. ProefschriftMaken Maastricht. https://doi.org/10.26481/dis.20191002ns

Document status and date:

Published: 01/01/2019

DOI:

10.26481/dis.20191002ns

Document Version:

Publisher's PDF, also known as Version of record

\section{Please check the document version of this publication:}

- A submitted manuscript is the version of the article upon submission and before peer-review. There can be important differences between the submitted version and the official published version of record. People interested in the research are advised to contact the author for the final version of the publication, or visit the DOI to the publisher's website.

- The final author version and the galley proof are versions of the publication after peer review.

- The final published version features the final layout of the paper including the volume, issue and page numbers.

Link to publication

\footnotetext{
General rights rights.

- You may freely distribute the URL identifying the publication in the public portal. please follow below link for the End User Agreement:

www.umlib.nl/taverne-license

Take down policy

If you believe that this document breaches copyright please contact us at:

repository@maastrichtuniversity.nl

providing details and we will investigate your claim.
}

Copyright and moral rights for the publications made accessible in the public portal are retained by the authors and/or other copyright owners and it is a condition of accessing publications that users recognise and abide by the legal requirements associated with these

- Users may download and print one copy of any publication from the public portal for the purpose of private study or research.

- You may not further distribute the material or use it for any profit-making activity or commercial gain

If the publication is distributed under the terms of Article $25 \mathrm{fa}$ of the Dutch Copyright Act, indicated by the "Taverne" license above, 
Effect of Intradialytic Aerobic Exercise among Hemodialysis

Patients in the United Arab Emirates on Clinical Outcomes, Barriers to Physical Activity, and Quality of Life

Nada Salhab

2019 
The research presented in this thesis was conducted in the dialysis unit at Al Qassimi Hospital, Sharjah, in the United Arab Emirates. This study was performed in scientific collaboration with the School of Nutrition and Translational Research in Metabolism, Maastricht University, Maastricht, The Netherlands.

The project was funded by Zayed University.

Layout cover by: ProefschriftMaken || www.proefschriftmaken.nl

Printed by: ProefschriftMaken || www.proefschriftmaken.nl

ISBN: 978-94-6380-411-0

Copyright (C) Nada Salhab, 2019

No parts of this thesis may be reproduced, stored in a retrieved system, or transmitted, in any forms or by any means, electronically, mechanically, by photocopying, recording or otherwise, without the prior written permission of the author. For articles published or accepted, the copyright has been transferred to the respective publisher. 


\title{
Effect of Intradialytic Aerobic Exercise among Hemodialysis Patients in the United Arab Emirates on Clinical Outcomes, Barriers to Physical Activity, and Quality of Life
}

\author{
Dissertation \\ to obtain the degree of Doctor at Maastricht University, \\ on the authority of the Rector Magnificus Prof.dr. Rianne M. Letschert \\ in accordance with the decision of the Board of Deans, \\ to be defended in public on Wednesday $2^{\text {nd }}$ of October, 2019 \\ at 12:00 hours
}

by

Nada Salhab 


\section{Supervisor}

Prof. dr. J. P. Kooman

\section{Co-Supervisors}

Dr. M. Karavetian, Zayed University, Dubai, United Arab Emirates Prof. dr. E. Fiaccadori, Parma University, Parma, Italy

\section{Assessment Committee}

Prof. dr. A.E.R.C.H. Boonen (chair)

Prof. dr. N.K. de Vries

Prof. dr. S.M. Evers

Dr. C.J. Konings, Catharina Hospital, Eindhoven, Netherlands

Prof. dr. P.M. ter Wee, Free University Medical Center, Amsterdam, Netherlands 


\section{Content}

$\begin{array}{lll}\text { Chapter } 1 \text { General Introduction } & 7\end{array}$

Chapter 2 Effect Of Intradialytic Aerobic Exercise On Hemodialysis 25

Patients: A Systematic Review And Meta - Analysis

Chapter 3 Intradialytic Aerobic Exercise In The United Arab Emirates: A 59 Descriptive Study

Chapter 4 Effects Of Intradialytic Aerobic Exercise On Hyperphosphatemia 81 And Health Outcomes In Hemodialysis Patients

Chapter 5 Attitudes And Barriers To Physical Activity In Hemodialysis Patients: Could Intradialytic Exercise Modify These Factors

Chapter 6 Malnutrition inflammation score is better diagnostic tool than phase angle when compared to glim criteria among hemodialysis patients - results from a cross sectional study

Chapter 7 General Discussion

Study Summary

Valorization

Publications

Acknowledgements

Curriculum vitae 

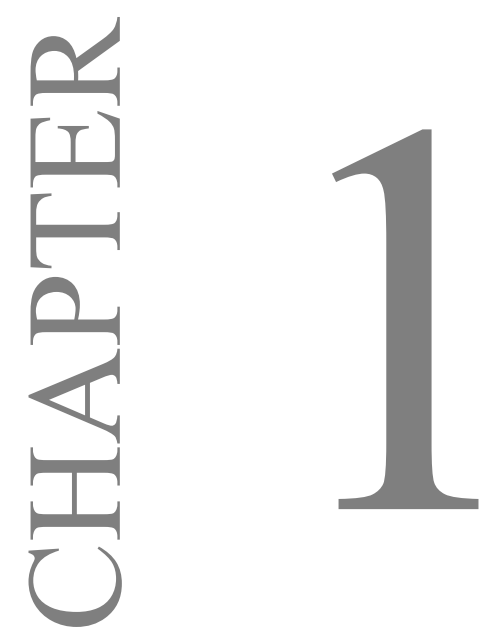

GENERAL INTRODUCTION 
The kidneys' function is to maintain the body homeostatic balance. On average, the kidneys produce daily 180 liters of fluid through blood filtration, and excrete around 1.5 liters of urine after active reabsorption and secretion. Chronic kidney disease (CKD) leads to a disruption of these essential functions. CKD is divided into stages, depending on the severity of the disease; the most life changing stage for the patient is stage 5 or end stage renal disease (ESRD) (K/DOQI, 2002). In ESRD, the kidneys have almost lost all their ability to excrete waste products, maintain fluid and electrolyte balance, and produce hormones. To survive in this advanced stage, patients should opt for renal replacement therapy (RRT), which has 2 main branches, dialysis and transplantation (Mahan \& Stump, 2000). Dialysis has 3 main modalities: in-center hemodialysis, home hemodialysis, and peritoneal dialysis (Robinson et al., 2016). In hemodialysis, the artificial kidney, i.e., filter or dialyzer, is composed of several thousand hollow fibers approximating a surface of $0.5-2 \mathrm{~m}^{2}$, which separate the patient's blood from the dialysis fluid. Blood is pumped through the dialyzer where solutes are removed by diffusion and convection (Crawford \& Lerma, 2008), while excess water and sodium are removed by ultrafiltration, a process based on pressure differences created by the dialysis machine between the blood and dialysate compartment. Unfortunately, dialysis is not a definitive treatment for ESRD patients, and despite adequate prescription, the progression of the disease could lead to various complications (gastrointestinal, neurologic, hematologic, cardiovascular), bone disease, electrolyte disturbances, thus affecting every organ system (Obrador \& Pereira, 2002).

\section{PREVALENCE OF CKD: WORLDWIDE AND IN THE UNITED ARAB EMIRATES}

CKD prevalence is estimated to be $13.4 \%$ worldwide (Hill et al., 2016), hence, a growing global public health concern (Eckardt et al., 2013). In the UAE, CKD prevails among nationals in $4.6 \%$ of the males and $2.8 \%$ of the females. Similar percentages were recorded for expatriates residing in the UAE, with $4.2 \%$ of male and $3.2 \%$ of females suffering from CKD (Richards et al., 2015). In the UAE, the treatment of ESRD with hemodialysis started in 1977, and today, there are 33 dialysis centers offering this treatment for 1750 patients. The descriptive characteristics of the latter were defined as follows: $40 \%$ are citizens, $93 \%$ are hypertensive and 55\% of male gender, with diabetes (34\%) and hypertension (39\%) being the most common causes for HD initiation. The annual mortality rate of the total dialysis population in the UAE is $8.5 \%$ (AlSahow et al., 2016). 


\section{SEDENTARISM IN HD PATIENTS: A MODIFIABLE RISK FACTOR?}

HD patients are often drifted to a sedentary lifestyle, which is associated with an increased risk of mortality (O’Hare et al., 2003, Stack et al., 2005, Tentori et al., 2010). Major CKD manifestations that contribute to the vicious cycle of decreased physical functioning and reduced physical activity are metabolic acidosis, ureamia, inflammation and malnutrition (Zelle et al., 2017). In fact, physical activity in HD patients is lower than in healthy sedentary controls who reported exercising a maximum of once a week (Johansen et al., 2000). In a multicenter study, the mean number of steps recorded in HD patients was lower that the set $<7500$ steps/day matching a sedentary to low active lifestyle (Avesani et al., 2012).

Researchers are constantly looking for the appropriate methods to tackle the sedentarism problem, and studying the barriers to physical activity (PA) was one of them. Unfortunately, HD patients face various barriers to PA. Based on a recent review, HD patients' most common barriers to PA are: fatigue and lack of energy, comorbidities and lack of time and access (Hannan \& Bronas, 2017). Equally important was the lack of motivation coupled with the lack of time faced by the medical team as major factors for patients not to exercise (Goodman \& Ballou, 2004; Jhamb et al., 2006; Fiaccadori et al., 2014; Regolisti et al., 2018). On the other hand, multiple benefits were recorded in HD patients who reported to engage in physical activity; these include higher quality of life (QOL) (Lopes et al., 2014; Tentori et al., 2010), physical functioning and sleep quality scores (Tentori et al., 2010). The Kidney Disease Improving Global Outcomes (KDIGO) guidelines recommend for CKD patients to exercise at least 30 minutes per day, 5 times a week (Ketteler et al., 2017). Such recommendations tailored to HD population are still missing. It is worth mentioning that data from DOPPS including 20920 patients from 12 countries showed that more patients tend to exercise in facilities offering exercise programs (Tentori et al., 2010); thus, efforts to counteract sedentarism may be beneficial.

\section{HYPERPHOSPHATEMIA IN HD PATIENTS: A SILENT KILLER}

Elevated serum phosphorus (P) levels (hyperphosphatemia) is another major risk factor for mortality in HD patients (Kuhlmann et al., 2006; Quniby, 2004), and prevails in almost 50\% of this population (Young et al., 2004). The exact mechanism of why hyperphosphatemia augments the mortality risks is still unclear, but it might mainly relate to cardiovascular calcification (Quniby, 2004). Hyperphosphatemia results from the loss of phosphorus homeostasis due to the impaired kidney function, but its regulation is complex. The body has various defense mechanisms against hyperphosphatemia; these include the actions of both the fibroblast growth factor 23 (FGF-23) and the parathyroid hormone (PTH) that inhibit tubular 
phosphate reabsorption; however, with the deteriorated glomerular filtration rate in patients with advanced CKD, FGF-23 and PTH fail to regulate serum P levels (Hruska et al., 2008). In addition, increased FGF-23 has been associated with pathology itself, contributing to cardiovascular problems (Scialla \& Wolf, 2014), and independently associated with mortality (Gutierrez et al., 2008; Isakova et al., 2018). Another body defense mechanism against hyperphosphatemia is the formation of calciprotein particles (CPP), a complex of calcium, phosphorus complex and proteins such as fetuin, initially preventing vascular calcification. However, it is suspected that in CKD patients, the increased production of CPP or their decreased clearance could exacerbate the vascular calcification (Paloian \& Giachelli, 2014). Next to the loss of kidney function, a major contributor to hyperphosphatemia is the increased bone resorption compared to bone formation rates (Hruska et al., 2008). KDIGO guidelines recommend lowering serum phosphorus $(\mathrm{P})$ level towards the normal range of healthy populations equivalent to $2.5-4.5 \mathrm{mg} / \mathrm{dl}$ (Ketteler et al., 2017). In an attempt to achieve the recommended level, the clinical management of hyperphosphatemia relies on: a) routine dialysis, which cannot clear all the excess $\mathrm{P}$; b) low $\mathrm{P}$ diet, which can lead to protein energy malnutrition (Ikizler, 2009); and c) intake of phosphate binders which can cause gastrointestinal side effects (St. Peter et al., 2017), and adds cost on the patient and the healthcare system (White et al., 2006). In addition, the elevated number of phosphate binder pills is a major determinant in lowering the QOL in dialysis patients (Chiu et al., 2009). Despite these available treatment modalities, reaching the patient's therapeutic target for $\mathrm{P}$ is not always successful. Thus, rises the necessity for an adjunct therapy targeting hyperphosphatemic HD patients.

\section{QUALITY OF LIFE IN HD PATIENTS: PHYSIOLOGY SHAPING PSYCHOLOGY}

HD is a life-changing process requiring the ESRD patient to visit the dialysis unit usually 3 times per week for 3-5 hours (Mahan \& Stump, 2000). As one would expect, this lifestyle change diminishes the QOL of these patients as compared to healthy adult (Avramovic \& Stefanovic, 2012); in fact, the more advance the CKD stage, the more impaired the QOL (Aggarwal et al., 2016). In addition, patient on dialysis have lower QOL than non-dialysis CKD patients. A study conducted in the UAE at the Sheikh Khalifa Medical City about the QOL of dialysis patients showed that patients with ongoing chronic health problems had lower QOL scores (Ayoub \& Hijjazy, 2013). As QOL could significantly predict patient's survival and hospitalization rate (Avramovic \& Stefanovic, 2012), any intervention that could positively impact QOL is worth to investigate. 


\section{INTRADIALYTIC EXERCISE: AN INNOVATIVE STEP IN RENAL CARE}

Intradialytic exercise (IDE) is described as training performed during the HD session. There are several used IDE modalities including resistance, aerobic and stretching exercises. The most used tools in IDE are cycle ergometers, stretch bands and exercise balls. Researchers are still unsure about the exact mechanism behind IDE; it is hypothesized that, during IDE, more toxins are removed through the dialyzer due to the increase in blood flow and capillary vasodilation (Maheshwari et al., 2011). In fact, studies have shown that IDE ameliorates solute clearance in general (Sheng et al., 2014) and phosphorus removal in particular (Musavian et al., 2015; Makhlough et al., 2012). In addition, IDE positive effect on QOL was evident in the literature (Musavian et al., 2015; Reboredo et al., 2010; Giannaki et al., 2013; Golebiowski et al., 2012; Wu et al., 2014). Last but not least, IDE resulted in a positive effect on the overall health (de Lima et al., 2013), paralleled with a non-significant decrease in hospitalization rate in HD patient (Parker et al., 2015). Despite the fact that IDE has shown to have physiological and psychological positive effects in HD patients, it is not included in HD patient treatment protocol. To date, few dialysis units offer the "luxury" of IDE to their patients; a major contributor to this fact is the lack of guidelines for IDE. The lack of sufficient evidence on IDE safety, effectiveness and cost effectiveness position it as a frail skeleton (Sheng et al., 2014; Chung et al., 2017), and patients considers this experience as "going to the unknown" (Young et al., 2015).

\section{MALNUTRITION ASSESSMENT IN HD PATIENTS}

Malnutrition is a prevalent condition in HD patients and often coincides with inflammation leading to the malnutrition inflammation syndrome (Sabatino et al., 2017). This syndrome is associated with frailty, depression, higher morbidity and mortality, and decreased quality of life (Ikizler et al., 2013; Sabatino et al., 2017). Thus, early assessment of malnutrition in this population becomes crucial. The facts that body composition is in constant change in HD patients (Chazot et al., 2014) and renal dietitians face time limitation in their work (Wolfe, 2012), give rise to the need of a user friendly and accurate tool for malnutrition assessment. The available diagnostic tools range from anthropometric, to biochemical parameters, technical investigations, and various composite score (Rogowski et al., 2018; da silva et al., 2018; Garcia et al., 2013). Recently, the Global Initiative on Malnutrition (GLIM) presented updated criteria for the diagnosis of malnutrition (Cederholm et al., 2018). The GLIM assessment criteria for malnutrition included phenotypic criteria, which are: 1) non-volitional weight loss (weight loss of $>5 \%$ within the past 6 month), 2) low body mass index ( $<20$ $\mathrm{kg} / \mathrm{m}^{2}$ if $<70$ years, $<22 \mathrm{~kg} / \mathrm{m}^{2}$ if $\geq 70$ years), 3 ) reduced muscle mass (Fat free mass index 
$<17 \mathrm{~kg} / \mathrm{m}^{2}$ for males and $<15 \mathrm{~kg} / \mathrm{m}^{2}$ for females) (Cederholm et al., 2015) and two etiologic criteria which are reduced food intake or assimilation and inflammation or disease burden. In order to diagnose malnutrition at least one phenotypic and one etiologic criterion should be present. It is still not reported whether the available diagnostic tools are in accordance with the GLIM criteria.

Today, many dialysis units rely on the Malnutrition-Inflammation Score (MIS) for the diagnosis of malnutrition (Kalantar-Zadeh et al., 2001; Pisetkul et al., 2010; Rambod et al., 2009). However, MIS is time consuming, requiring blood tests, and a certain level of expertise in this field. Recently, bioelectrical impedance analysis (BIA)-derived phase angle $(\mathrm{PhA})$ is being studied, as it might be a practical, cost-effective tool for the assessment of malnutrition. The $\mathrm{PhA}$ is proportional to the ratio of reactance and resistance between body cell mass and fat-free mass (Baumgartner et al., 1988), and is assumed to indicate cell integrity (Barbosa \& Barros, 2005). In various clinical fields, PhA was shown to be reliable marker for the detection of early malnutrition (Kyle et al., 2012; Wiech et al., 2018). Thus, any attempt to investigate or validate available $\mathrm{PhA}$ cut-off points is of potential advantage.

\section{STUDY OBJECTIVES}

This is the first study to introduce IDE in the routine practice of HD patients in the UAE. The major aim of the thesis was to investigate whether a 6-month supervised aerobic IDE program has an effect on HD patients' exercising behavior and attitude, QOL, barriers to exercise, in addition to clinical parameters including serum $\mathrm{P}$, parathyroid hormone (PTH) levels, dialysis efficiency, nutritional status in the UAE.

Next to this, and in order to develop our intervention protocol, we aimed for a meta-analysis to have firmer conclusions to base our protocol on, especially that the sample size in most available studies was small.

Last, we aimed to assess malnutrition on a larger sample in the same dialysis unit, and compare the concordance of the MIS and BIA - derived PhA with the GLIM criteria for the diagnosis of malnutrition. 
Study objectives:

Conduct a meta-analysis to base our protocol on its findings

$>$ Assess the baseline characteristics of HD patients in the UAE. These included clinical and non-clinical parameters as follows:

- Demographics

- Quality of life

- Attitude, behavior and knowledge towards exercise

- Barriers to physical activity

- Hyperphosphatemia

- Dialysis efficiency

- Malnutrition inflammation score (MIS)

$>$ Examine the effect of a 6-month aerobic IDE program on the above cited parameters.

Assess the medical team's attitudes towards exercise in HD patients.

$>$ Determine whether the MIS and the BIA derived PhA are in accordance with the GLIM criteria for the diagnosis of malnutrition in HD patients.

\section{THESIS OUTLINE}

Chapter 1 presents a general introduction about the topic.

Chapter 2 details the systematic review and meta-analysis of published aerobic IDE interventions. It tabulates the effects of aerobic IDE on patient $Q O L, P$, dialysis efficiency [Urea Reduction Ratio (URR), Kt/V], vitamin D3, PTH, and serum C-reactive protein (CRP), intake of $P$ binders, the number of emergency $H D$, cost effectiveness, mortality and hospitalization rate.

This review adds to previously published review the serum phosphorus as a new studied parameter. It also focused on the effects of aerobic IDE since combined exercise regimens are usually more complex to implement in a dialysis unit.

The search was conducted using Pubmed, Medline (Ovid), Embase (Ovid), Cochrane, and Cinahl (EBSCO) databases. Results included 22 studies evaluating the effect of IDE on QOL, serum $\mathrm{P}$, dialysis efficiency, C-reactive protein, and hospitalization rate. Unfortunately, none of the screened articles measured the other parameters that we initially planned to assess, thus 
they were omitted from the results. A putative recipe for aerobic IDE was formulated at the end of the review.

Table 1 Putative recipe for aerobic IDE

\begin{tabular}{ll}
\hline Preconditioning & $\begin{array}{l}\text { Strength training and stretching exercises } \\
2 \text { months prior to exercise initiation }\end{array}$ \\
Modality & Warm up: Range capacity exercise \\
& + Cycling \\
Frequency & 3 times per week \\
Intensity & Borg scale average rating of 12 points \\
& Or $60-65 \%$ of the peak power output \\
Duration & Range capacity exercises: 15 minutes \\
& + Cycling: 30 - 45 minutes \\
\hline
\end{tabular}

Chapter 3 describes the study protocol, and presents the baseline characteristics of all patients that were eligible to enter an IDE program in the largest HD unit in Sharjah emirates, in the UAE. Some of these characteristics were assessed for the first time in the $U A E$.

The intervention was based on the results of the systematic review, and was coherent with the formulated putative recipe for aerobic IDE. Based on the set objectives, the study design was developed (Figure 1.1). 
- Training of the recruited research assistants

- Validation of the questionnaires

- Verification of the methods

- Patient education on the importance of exercise

- Baseline $\left(\mathrm{T}_{0}\right)$ assessment on all study parameters

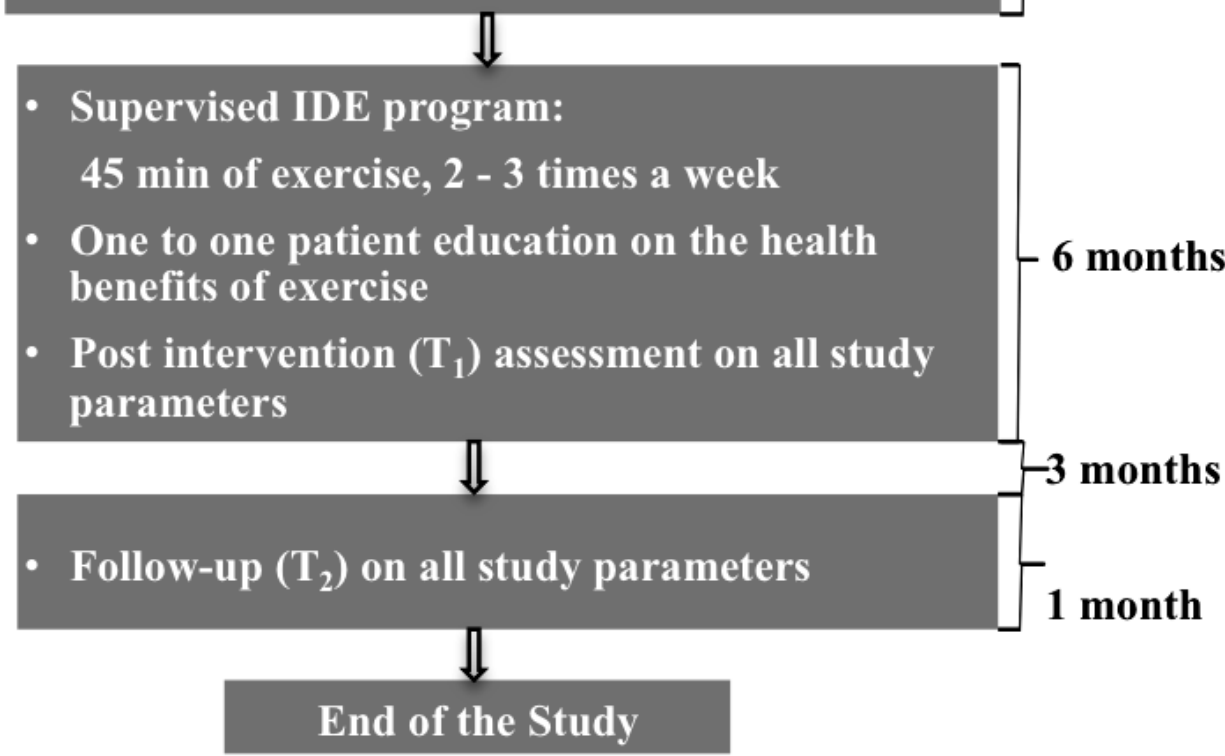

Figure 1.1: Flow Chart of the Study Design

Chapter 4 demonstrates the results of the effects of aerobic IDE on hyperphosphatemia, quality of life, dialysis efficiency and malnutrition inflammation score.

Hyperphosphatemia is proposed as a "silent killer in HD patients" (Amann et al., 1999). Its prevalence in the UAE has been recently reported to be $73.8 \%$ with a cut-off point of $4.5 \mathrm{mg} / \mathrm{dl}$ (Rabbani et al., 2017). This is the first trial in the UAE to implement IDE in an HD setting, and to present its effects on hyperphosphatemia, QOL, dialysis efficiency and MIS. 
Chapter 5 presents the effect of IDE on patient's behavior, attitude, knowledge and barriers to physical activity. In addition, the attitudes of the nephrologists and HD nurses towards exercise are presented in chapter 5.

This is the first study in the UAE that unveils the behavior, attitude, knowledge, and barriers to physical activity in HD patients, and compare the results cross culturally.

Chapter 6 highlights the importance of evaluating the malnutrition status in HD patients, which is a major determinant of their health.

Various tools are available to assess the nutritional status of hospitalized patients. In this thesis, we assessed malnutrition using the MIS and determined whether the easy to use bioelectrical analyzer derived phase angle and the MIS are in accordance with the new Global Initiative of Malnutrition (GLIM) criteria for the diagnosis of malnutrition in HD patients.

Chapter 7 presents a general discussion of the study results. 


\section{REFERENCES}

Aggarwal, H. K., Jain, D., Pawar, S., \& Yadav, R. K. (2016). Health-related quality of life in different stages of chronic kidney disease. Qjm, 109(11), 711-716.

doi:10.1093/qjmed/hcw054

AlSahow, A., AlRukhaimi, M., Al Wakeel, J., Al-Ghamdi, S. M., AlGhareeb, S., AlAli, F., . . . Pisoni, R. L. (2016). Demographics and key clinical characteristics of hemodialysis patients from the Gulf Cooperation Council countries enrolled in the dialysis outcomes and practice patterns study phase 5 (2012-2015). Saudi J Kidney Dis Transpl, 27(6 Suppl 1), S12-23. doi:10.4103/1319-2442.194885

Amann, K., Gross, M. L., London, G. M., \& Ritz, E. (1999). Hyperphosphataemia--a silent killer of patients with renal failure? Nephrol Dial Transplant, 14(9), 2085-2087.

Avesani, C. M., Trolonge, S., Deleaval, P., Baria, F., Mafra, D., Faxen-Irving, G., . . . Fouque, D. (2012). Physical activity and energy expenditure in haemodialysis patients: an international survey. Nephrol Dial Transplant, 27(6), 2430-2434. doi:10.1093/ndt/gfr692

Avramovic, M., \& Stefanovic, V. (2012). Health-related quality of life in different stages of renal failure. Artif Organs, 36(7), 581-589. doi:10.1111/j.1525-1594.2011.01429.x

Ayoub, A. M., \& Hijjazi, K. H. (2013). Quality of life in dialysis patients from the United Arab Emirates. J Family Community Med, 20(2), 106-112. doi:10.4103/22308229.114772

Barbosa-Silva, MCG (2008|). Subjective and Objective Nutritional assessment methods: what do they really assess? Curr Opin Clin Nutr Metab Care, 11(3), 248-54.

Baumgartner, R. N., Chumlea, W. C., \& Roche, A. F. (1988). Bioelectric impedance phase angle and body composition. Am J Clin Nutr, 48(1), 16-23. doi:10.1093/ajcn/48.1.16

Cederholm, T., Bosaeus, I., Barazzoni, R., Bauer, J., Van Gossum, A., Klek, S., . . Singer, P. (2015). Diagnostic criteria for malnutrition - An ESPEN Consensus Statement. Clin Nutr, 34(3), 335-340. doi:10.1016/j.clnu.2015.03.001

Cederholm, T., Jensen, G. L., Correia, M., Gonzalez, M. C., Fukushima, R., Higashiguchi, T., ... Compher, C. (2018). GLIM criteria for the diagnosis of malnutrition - A consensus report from the global clinical nutrition community. Clin Nutr. doi:10.1016/j.clnu.2018.08.002 
Chazot, C., Deleaval, P., Bernollin, A. L., Vo-Van, C., Lorriaux, C., Hurot, J. M., . . Jean, G. (2014). Target weight gain during the first year of hemodialysis therapy is associated with patient survival. Nephron Clin Pract, 126(3), 128-134. doi:10.1159/000362211

Chiu, Y. W., Teitelbaum, I., Misra, M., de Leon, E. M., Adzize, T., \& Mehrotra, R. (2009). Pill burden, adherence, hyperphosphatemia, and quality of life in maintenance dialysis patients. Clin J Am Soc Nephrol, 4(6), 1089-1096. doi:10.2215/cjn.00290109

Chung, Y. C., Yeh, M. L., \& Liu, Y. M. (2017). Effects of intradialytic exercise on the physical function, depression and quality of life for haemodialysis patients: a systematic review and meta-analysis of randomised controlled trials. J Clin Nurs, 26(13-14), 1801-1813. doi:10.1111/jocn.13514

Crawford, P. W., \& Lerma, E. V. (2008). Treatment options for end stage renal disease. Prim Care, 35(3), 407-432, v. doi:10.1016/j.pop.2008.05.003

da Silva, A. T., Hauschild, D. B., Moreno, Y. M. F., Bastos, J. L. D., \& Wazlawik, E. (2018). Diagnostic Accuracy of Bioelectrical Impedance Analysis Parameters for the Evaluation of Malnutrition in Patients Receiving Hemodialysis. Nutr Clin Pract. doi:10.1002/ncp. 10098

de Lima, M. C., Cicotoste Cde, L., Cardoso Kda, S., Forgiarini, L. A., Jr., Monteiro, M. B., \& Dias, A. S. (2013). Effect of exercise performed during hemodialysis: strength versus aerobic. Ren Fail, 35(5), 697-704. doi:10.3109/0886022x.2013.780977

Eckardt, K. U., Coresh, J., Devuyst, O., Johnson, R. J., Kottgen, A., Levey, A. S., \& Levin, A. (2013). Evolving importance of kidney disease: from subspecialty to global health burden. Lancet, 382(9887), 158-169. doi:10.1016/s0140-6736(13)60439-0

Fiaccadori, E., Sabatino, A., Schito, F., Angella, F., Malagoli, M., Tucci, M., . . Regolisti, G. (2014). Barriers to physical activity in chronic hemodialysis patients: a singlecenter pilot study in an Italian dialysis facility. Kidney Blood Press Res, 39(2-3), 169175. doi:10.1159/000355793

Garcia, M.F., Wazlawik, E., Franco Moreno, Y.M., Fhur, L.M, Gonzalez-Chica, D.A. (2013). Diagnostic accuracy of handgrip strength in the assessment of malnutrition in hemodialyzed patients. e-SPEN Journal , 8( 4), (e181 - e186). doi:10.1016/j.clnme.2013.06.003

Giannaki, C. D., Sakkas, G. K., Karatzaferi, C., Hadjigeorgiou, G. M., Lavdas, E., Kyriakides, T., ... Stefanidis, I. (2013). Effect of exercise training and dopamine 
agonists in patients with uremic restless legs syndrome: a six-month randomized, partially double-blind, placebo-controlled comparative study. BMC Nephrol, 14, 194. doi:10.1186/1471-2369-14-194

Golebiowski, T., Kusztal, M., Weyde, W., Dziubek, W., Wozniewski, M., Madziarska, K., . . . Klinger, M. (2012). A program of physical rehabilitation during hemodialysis sessions improves the fitness of dialysis patients. Kidney Blood Press Res, 35(4), 290 296. doi: $10.1159 / 000335411$

Goodman, E. D., \& Ballou, M. B. (2004). Perceived barriers and motivators to exercise in hemodialysis patients. Nephrol Nurs J, 31(1), 23-29.

Gutierrez, O. M., Mannstadt, M., Isakova, T., Rauh-Hain, J. A., Tamez, H., Shah, A., . . Wolf, M. (2008). Fibroblast growth factor 23 and mortality among patients undergoing hemodialysis. N Engl J Med, 359(6), 584-592. doi:10.1056/NEJMoa0706130

Hannan, M., \& Bronas, U. G. (2017). Barriers to exercise for patients with renal disease: an integrative review. J Nephrol, 30(6), 729-741. doi:10.1007/s40620-017-0420-z

Hill, N. R., Fatoba, S. T., Oke, J. L., Hirst, J. A., O'Callaghan, C. A., Lasserson, D. S., \& Hobbs, F. D. (2016). Global Prevalence of Chronic Kidney Disease - A Systematic Review and Meta-Analysis. PLoS One, 11(7), e0158765. doi:10.1371/journal.pone.0158765

Hruska, K. A., Mathew, S., Lund, R., Qiu, P., \& Pratt, R. (2008). Hyperphosphatemia of chronic kidney disease. Kidney Int, 74(2), 148-157. doi:10.1038/ki.2008.130

Ikizler, T. A. (2009). Dietary protein restriction in CKD: the debate continues. Am J Kidney Dis, 53(2), 189-191. doi:10.1053/j.ajkd.2008.12.014

Ikizler, T. A., Cano, N. J., Franch, H., Fouque, D., Himmelfarb, J., Kalantar-Zadeh, K., . . . Wanner, C. (2013). Prevention and treatment of protein energy wasting in chronic kidney disease patients: a consensus statement by the International Society of Renal Nutrition and Metabolism. Kidney Int, 84(6), 1096-1107. doi:10.1038/ki.2013.147

Isakova, T., Cai, X., Lee, J., Xie, D., Wang, X., Mehta, R., . . Wolf, M. (2018). Longitudinal FGF23 Trajectories and Mortality in Patients with CKD. J Am Soc Nephrol, 29(2), 579-590. doi:10.1681/asn.2017070772

Jhamb, M., McNulty, M. L., Ingalsbe, G., Childers, J. W., Schell, J., Conroy, M. B., . . Dew, M. A. (2016). Knowledge, barriers and facilitators of exercise in dialysis patients: a 
qualitative study of patients, staff and nephrologists. BMC Nephrol, 17(1), 192. doi:10.1186/s12882-016-0399-z

Johansen, K. L., Chertow, G. M., Ng, A. V., Mulligan, K., Carey, S., Schoenfeld, P. Y., \& Kent-Braun, J. A. (2000). Physical activity levels in patients on hemodialysis and healthy sedentary controls. Kidney Int, 57(6), 2564-2570. doi:10.1046/j.15231755.2000.00116.x

Kalantar-Zadeh, K., Kopple, J. D., Block, G., \& Humphreys, M. H. (2001). A malnutritioninflammation score is correlated with morbidity and mortality in maintenance hemodialysis patients. Am J Kidney Dis, 38(6), 1251-1263. doi:10.1053/ajkd.2001.29222

$\mathrm{K} / \mathrm{DOQI}$ clinical practice guidelines for chronic kidney disease: evaluation, classification, and stratification. (2002). Am J Kidney Dis, 39(2 Suppl 1), S1-266.

Ketteler M, Block GA, Evenepoel P, et al. Executive summary of the 2017 KDIGO Chronic Kidney Disease-Mineral and Bone Disorder (CKD-MBD) Guideline Update: what's changed and why it matters. Kidney Int. 2017;92:26-36. (2017). Kidney Int, 92(6), 1558. doi:10.1016/j.kint.2017.10.001

Kuhlmann, M. K. (2006). Management of hyperphosphatemia. Hemodial Int, 10(4), 338-345. doi:10.1111/j.1542-4758.2006.00126.x

Kyle, U. G., Soundar, E. P., Genton, L., \& Pichard, C. (2012). Can phase angle determined by bioelectrical impedance analysis assess nutritional risk? A comparison between healthy and hospitalized subjects. Clin Nutr, 31(6), 875-881. doi:10.1016/j.clnu.2012.04.002

Lopes, A. A., Lantz, B., Morgenstern, H., Wang, M., Bieber, B. A., Gillespie, B. W., . . Pisoni, R. L. (2014). Associations of self-reported physical activity types and levels with quality of life, depression symptoms, and mortality in hemodialysis patients: the DOPPS. Clin J Am Soc Nephrol, 9(10), 1702-1712. doi:10.2215/cjn.12371213

Mahan, L. K., \& Escott-Stump, S. (2000). Krause's food, nutrition, \& diet therapy (10th ed. ed.). Philadelphia: W.B. Saunders.

Maheshwari, V., Samavedham, L., \& Rangaiah, G. P. (2011). A regional blood flow model for beta2-microglobulin kinetics and for simulating intra-dialytic exercise effect. Ann Biomed Eng, 39(12), 2879-2890. doi:10.1007/s10439-011-0383-5 
Makhlough, A., Ilali, E., Mohseni, R., \& Shahmohammadi, S. (2012). Effect of intradialytic aerobic exercise on serum electrolytes levels in hemodialysis patients. Iran J Kidney Dis, 6(2), 119-123.

Musavian, A. S., Soleimani, A., Masoudi Alavi, N., Baseri, A., \& Savari, F. (2015). Comparing the effects of active and passive intradialytic pedaling exercises on dialysis efficacy, electrolytes, hemoglobin, hematocrit, blood pressure and healthrelated quality of life. Nurs Midwifery Stud, 4(1), e25922.

O'Hare, A. M., Tawney, K., Bacchetti, P., \& Johansen, K. L. (2003). Decreased survival among sedentary patients undergoing dialysis: results from the dialysis morbidity and mortality study wave 2. Am J Kidney Dis, 41(2), 447-454. doi:10.1053/ajkd.2003.50055

Obrador, G. T., \& Pereira, B. J. (2002). Systemic complications of chronic kidney disease. Pinpointing clinical manifestations and best management. Postgrad Med, 111(2), 115122; quiz 121.

Paloian, N. J., \& Giachelli, C. M. (2014). A current understanding of vascular calcification in CKD. Am J Physiol Renal Physiol, 307(8), F891-900.

doi:10.1152/ajprenal.00163.2014

Parker, K., Zhang, X., Lewin, A., \& MacRae, J. M. (2015). The association between intradialytic exercise and hospital usage among hemodialysis patients. Appl Physiol Nutr Metab, 40(4), 371-378. doi:10.1139/apnm-2014-0326

Pisetkul, C., Chanchairujira, K., Chotipanvittayakul, N., Ong-Ajyooth, L., \& Chanchairujira, T. (2010). Malnutrition-inflammation score associated with atherosclerosis, inflammation and short-term outcome in hemodialysis patients. J Med Assoc Thai, 93 Suppl 1, S147-156.

Qunibi, W. Y. (2004). Consequences of hyperphosphatemia in patients with end-stage renal disease (ESRD). Kidney Int Suppl(90), S8-s12. doi:10.1111/j.15231755.2004.09004.x

Rabbani, S. A., S, S. B., Rao, P. G., Kurian, M. T., \& Essawy, B. E. (2017). HYPERPHOSPHATEMIA IN END STAGE RENAL DISEASE: PREVALENCE AND PATIENTS CHARACTERISTICS OF MULTIETHNIC POPULATION OF UNITED ARAB EMIRATES. International Journal of Pharmacy and Pharmaceutical Sciences, 9(12), 283. doi:10.22159/ijpps.2017v9i12.22425 
Rambod, M., Bross, R., Zitterkoph, J., Benner, D., Pithia, J., Colman, S., . . Kalantar-Zadeh, K. (2009). Association of Malnutrition-Inflammation Score with quality of life and mortality in hemodialysis patients: a 5-year prospective cohort study. Am J Kidney Dis, 53(2), 298-309. doi:10.1053/j.ajkd.2008.09.018

Reboredo Mde, M., Henrique, D. M., Faria Rde, S., Chaoubah, A., Bastos, M. G., \& de Paula, R. B. (2010). Exercise training during hemodialysis reduces blood pressure and increases physical functioning and quality of life. Artif Organs, 34(7), 586-593. doi:10.1111/j.1525-1594.2009.00929.x

Regolisti, G., Maggiore, U., Sabatino, A., Gandolfini, I., Pioli, S., Torino, C., . . Fiaccadori, E. (2018). Interaction of healthcare staff's attitude with barriers to physical activity in hemodialysis patients: A quantitative assessment. PLoS One, 13(4), e0196313. doi:10.1371/journal.pone.0196313

Richards, N., Hassan, M., Saleh, A. K., Dastoor, H., Bernieh, B., Abouchacra, S., . . Marcelli, D. (2015). Epidemiology and referral patterns of patients with chronic kidney disease in the Emirate of Abu Dhabi. Saudi J Kidney Dis Transpl, 26(5), 10281034. doi:10.4103/1319-2442.164600

Robinson, B. M., Akizawa, T., Jager, K. J., Kerr, P. G., Saran, R., \& Pisoni, R. L. (2016). Factors affecting outcomes in patients reaching end-stage kidney disease worldwide: differences in access to renal replacement therapy, modality use, and haemodialysis practices. Lancet, 388(10041), 294-306. doi:10.1016/s0140-6736(16)30448-2

Rogowski, L., Kusztal, M., Golebiowski, T., Bulinska, K., Zembron-Lacny, A., Wyka, J., . . . Dziubek, W. (2018). Nutritional assessment of patients with end-stage renal disease using the MNA scale. Adv Clin Exp Med, 27(8), 1117-1123. doi:10.17219/acem/75604

Sabatino, A., Regolisti, G., Karupaiah, T., Sahathevan, S., Sadu Singh, B. K., Khor, B. H., . . . Fiaccadori, E. (2017). Protein-energy wasting and nutritional supplementation in patients with end-stage renal disease on hemodialysis. Clin Nutr, 36 (3), 663-671. doi:10.1016/j.clnu.2016.06.007

Scialla, J. J., \& Wolf, M. (2014). Roles of phosphate and fibroblast growth factor 23 in cardiovascular disease. Nat Rev Nephrol, 10(5), 268-278. doi:10.1038/nrneph.2014.49

Sheng, K., Zhang, P., Chen, L., Cheng, J., Wu, C., \& Chen, J. (2014). Intradialytic exercise in hemodialysis patients: a systematic review and meta-analysis. Am J Nephrol, 40(5), 478-490. doi:10.1159/000368722 
St. Peter, W. L., Wazny, L. D., Weinhandl, E., Cardone, K. E., \& Hudson, J. Q. (2017). A Review of Phosphate Binders in Chronic Kidney Disease: Incremental Progress or Just Higher Costs? Drugs, 77(11), 1155-1186. doi:10.1007/s40265-017-0758-5

Stack, A. G., Molony, D. A., Rives, T., Tyson, J., \& Murthy, B. V. (2005). Association of physical activity with mortality in the US dialysis population. Am J Kidney Dis, 45(4), 690-701.

Tentori, F., Elder, S. J., Thumma, J., Pisoni, R. L., Bommer, J., Fissell, R. B., . . Robinson, B. M. (2010). Physical exercise among participants in the Dialysis Outcomes and Practice Patterns Study (DOPPS): correlates and associated outcomes. Nephrol Dial Transplant, 25(9), 3050-3062. doi:10.1093/ndt/gfq138

White, A., Odedina, F., Xiao, H., Campbell, E., \& Segal, R. (2006). The Economic Burden of End-Stage Renal Disease with Hyperphosphatemia: A Study of Florida Medicaid. DISEASE MANAGEMENT AND HEALTH OUTCOMES, 14(2), 99-106.

Wiech, P., Dabrowski, M., Bazalinski, D., Salacinska, I., Korczowski, B., \& BinkowskaBury, M. (2018). Bioelectrical Impedance Phase Angle as an Indicator of Malnutrition in Hospitalized Children with Diagnosed Inflammatory Bowel DiseasesA Case Control Study. Nutrients, 10(4). doi:10.3390/nu10040499

Wolfe, W. A. (2012). Moving the issue of renal dietitian staffing forward. J Ren Nutr, 22(5), 515-520. doi:10.1053/j.jrn.2012.04.006

Wu, Y., He, Q., Yin, X., He, Q., Cao, S., \& Ying, G. (2014). Effect of individualized exercise during maintenance haemodialysis on exercise capacity and health-related quality of life in patients with uraemia. $J$ Int Med Res, 42(3), 718-727. doi:10.1177/0300060513509037

Young, E. W., Akiba, T., Albert, J. M., McCarthy, J. T., Kerr, P. G., Mendelssohn, D. C., \& Jadoul, M. (2004). Magnitude and impact of abnormal mineral metabolism in hemodialysis patients in the Dialysis Outcomes and Practice Patterns Study (DOPPS). Am J Kidney Dis, 44(5 Suppl 2), 34-38.

Young, H. M., Hudson, N., Clarke, A. L., Dungey, M., Feehally, J., Burton, J. O., \& Smith, A. C. (2015). Patient and Staff Perceptions of Intradialytic Exercise before and after Implementation: A Qualitative Study. PLoS One, 10(6), e0128995. doi:10.1371/journal.pone.0128995 
Zelle, D. M., Klaassen, G., van Adrichem, E., Bakker, S. J., Corpeleijn, E., \& Navis, G. (2017). Physical inactivity: a risk factor and target for intervention in renal care. Nat Rev Nephrol, 13(3), 152-168. doi:10.1038/nrneph.2016.187 

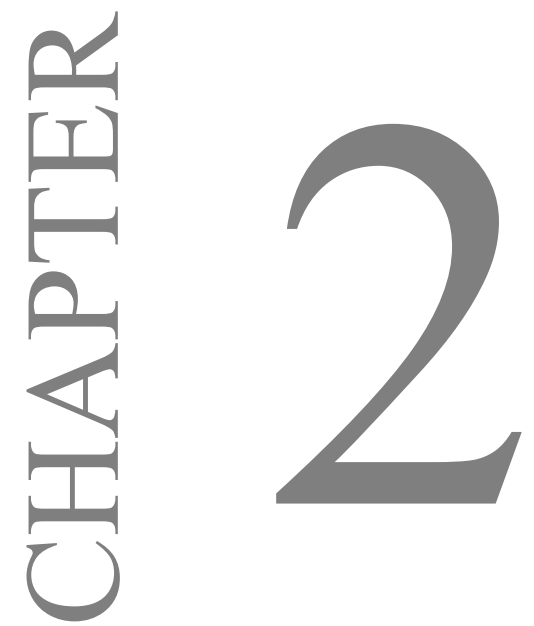

\section{EFFECTS OF INTRADIALYTIC AEROBIC EXERCISE ON HeModialysis Patients: A SySTEMATIC REVIEW AND META-ANALYSIS}

Salhab N, Karavetian M, Kooman J, Fiaccadori E, El Khoury C

Journal of Nephrology, 2018 [doi.org/10.1007/s40620-018-00565-z] 


\section{ABSTRACT}

Objective: Intradialytic exercise (IDE) is not yet a routine practice for hemodialysis patients, the lack of guidelines supporting is a major contributor to this fact. This systematic review and metaanalysis of aerobic IDE interventions examines the efficacy of IDE on quality of life (QOL), serum phosphorus, dialysis efficiency, inflammatory status, vitamin D3, parathyroid hormone, intake of phosphate binders, mortality and hospitalization rate.

Methods: Pubmed, Medline (Ovid), Embase (Ovid), Cochrane, and Cinahl (EBSCO) databases were searched to retrieve studies up to June 12, 2018; manual reference search was also performed. Studies were included if they evaluate (a) aerobic IDE on 1 of our studies parameters (b) in adult hemodialysis patients, (c) for a duration of $>1$ month.

Results: Twenty-two studies were included (706 participants) in the systematic review, out of which 12 were eligible for meta-analysis. Aerobic IDE had a significant positive effect on the physical component score QOL-PSC and on the mental component score QOL-MCS of SF36, but not on serum phosphorus and $\mathrm{Kt} / \mathrm{V}$.

Conclusions: IDE incorporation into clinical practice has a significant positive effect on QOL-PSC and QOL-MCS. In the reviewed studies, IDE did not result in any health hazard in hemodialysis patients; however, future research should assess long-term effectiveness and safety of IDE. The limitations of this review include the lack of quality analysis of the studies, the limited number of studies that could be included in the meta-analysis, the diversity in the exercise intensity, duration and modality, and the limited data for several outcomes.

Prospero Registration ID: CRD42016052062

Keywords: Hemodialysis, Intradialytic exercise, Quality of life, Phosphorus, Meta-Analysis 
Intradialytic exercise (IDE) is defined as exercise training programs during the hemodialysis (HD) session aiming to increase strength and endurance of HD patients and hence targeting various physiological and psychosocial parameters. The nature of the IDE varies between resistance, aerobic and stretching, using different equipment. IDE has shown to have a positive effect on the overall health and hospitalization rate of HD patients (de Lima et al., 2013; Parker et al., 2015).

Kidney Disease Improving Global Outcomes (KDIGO) recommends full integration of exercise within daily life of chronic kidney patients (at least 30 minutes per day, 5 times a week) taking into consideration their cardiovascular health and tolerance (Levin \& Stevens, 2014). Unfortunately, there are no clear guidelines for IDE, and from the patient's perspective, they describe IDE experience as "going into the unknown" (Young et al., 2015).

Sedentary lifestyle, low quality of life and reduced $\mathrm{VO}_{2}$ max are associated with increased mortality risk among HD patients (O'hare et al., 2003; Sietsema et al., 2004; van Loon et al. 2017). IDE has shown to have positive effects in HD patients; this was evident from the results of previous systematic reviews where IDE improved efficiency of dialysis (Kt/V) (Sheng et al., 2014; Cheema \& Singh, 2005), $\mathrm{VO}_{2}$ max (Sheng et al., 2014; Cheema \& Singh, 2005; Chung et al., 2017), and quality of life (QOL) (Sheng et al., 2014; Cheema \& Singh, 2005; Chung et al., 2017; Smart \& Steele, 2011). In addition, several studies have also shown positive effects of IDE on solute clearance, notably with regard to serum phosphorus levels (P) (de Lima et al., 2013; Makhlough et al., 2012; Reboredo et al., 2010; Musavian et al., 2015). Possible explanations of the underlying mechanism are the increased cardiac output and blood flow to lower extremities, capillary vasodilation, resulting in more solutes being transferred to the vascular compartment and reaching the dialyzer membrane for diffusion (Maheshwari et al., 2011). Thus IDE could be a cornerstone to the management of HD patients. .

Capitanini et al. (2014) suggested that, in order to sustain an exercise program for HD patients, a nephrologist should lead a team including a cardiologist, physiotherapist, exercise physiologist, renal dietitian, and a nurse. To date, some dialysis units are lucky enough to have ergometers that connect to the HD beds or chairs thus allowing their HD patients to benefit from this IDE aerobic exercise. Furthermore, exercise prescription for several chronic conditions (de Souto, 2017) is in alignment with the current international physical activity (PA) guidelines which is 150 minutes per week of moderate intensity aerobic PA (World Health Organization, 2010). Even though other recommendations include resistance training, this review focused on the effects of aerobic IDE since combined training is more complex for HD patients. 
The current review adds serum phosphate $(\mathrm{P})$ as new studied parameter in comparison to existing reviews, since it is an important risk factor associated with negative outcomes among HD patients (Tentori et al. 2008). The objective of this review is to explore all published interventions on aerobic IDE, identify the optimal protocols used, highlight the effects of these trials on patient QOL, P, dialysis efficiency [Urea Reduction Ratio (URR), $\mathrm{Kt} / \mathrm{V}$ ], vitamin D3, PTH, and serum C-reactive protein (CRP), intake of P binders, the number of emergency $\mathrm{HD}$, cost effectiveness, mortality and hospitalization rate.

\section{METHOD}

\section{Eligibility criteria}

All eligible studies were included if they met the predetermined inclusion and exclusion criteria detailed in the PICOS model (Table 1). There was no language restriction, nor filters applied to the search.

\section{Table 1 Eligibility Criteria}

\begin{tabular}{|c|c|c|}
\hline Criteria & Inclusion criteria & Exclusion criteria \\
\hline Population & - Chronic adult HD patients & - All other patients \\
\hline Intervention & - Aerobic IDE & $\begin{array}{l}\text { - } \quad \text { Non aerobic exercise } \\
\text { - Non-IDE intervention }\end{array}$ \\
\hline Comparison & $\begin{array}{l}\text { - } \quad \text { Pre - post intervention } \\
\text { - Intervention - control group }\end{array}$ & \\
\hline Outcomes & $\begin{array}{l}\text { - } \mathrm{QOL} \\
\text { - } \mathrm{Serum} \mathrm{P} \\
\text { - } \mathrm{Kt} / \mathrm{v} \\
\text { - } \mathrm{URR} \\
\text { - } \mathrm{Vitamin} \mathrm{D3} \\
\text { - } \mathrm{PTH} \\
\text { - } \mathrm{CRP} \\
\text { - Intake of P binders } \\
\text { - Number of emergency HD } \\
\text { - } \text { Mortality } \\
\text { - Hospitalization rate }\end{array}$ & \\
\hline Study types & - Interventional studies & $\begin{array}{ll}\text { - } & \text { Interventions }<1 \text { month } \\
\text { - } & \text { Non-interventional trials } \\
\text { - } & \text { Conference abstracts } \\
\text { - } & \text { Systematic reviews } \\
\text { - } & \text { Meta-analyses }\end{array}$ \\
\hline
\end{tabular}




\section{Search Strategy}

Potential studies were identified using the Pubmed, Medline (Ovid), Embase (Ovid), Cochrane, and Cinahl (EBSCO) databases on June 12, 2018. Furthermore, references of included articles were hand-searched by 2 reviewers; pertinent references were retrieved and subjected to full text screening. The search strategy included the following terms selected from the Medical Subject Headings (MeSH): renal replacement therapy, renal dialysis, hemodiafiltration, hemodialysis / home, renal insufficiency, renal osteodystrophy, dialysis, kidneys / artificial, motor activity, exercise, walking, physical exertion, physical endurance, exercise tolerance, physical fitness, exercise therapy, sports, bicycling, psychomotor performance, motor skills; it also included the following keywords: renal, kidney, insufficien*, replacement*, failure*, osteodytroph*, necrosis, necroses, artificial, dialy*, microdialy*, haemodialy*, hemodialy*, intradialy*, hemodiafiltration*, haemodiafiltration*, hemo-diafiltration*, haemo-diafiltration*, cardio, muscle*, muscular, endurance, aerobic*, physical, training, capacity*, capab*, therap*, toleran*, prescri*, interven*, techni*, physical*, motion*, activit*, fit, fitness, function*, exert*, modalit*, motor, skill*, psychomotor, performance. The asterisk $(*)$ sign denotes that the word before it will be searched in all its possible versions. Boolean operators "OR, AND" were used for the search. The full search strategy is available on https://www.crd.york.ac.uk/PROSPEROFILES/52062_STRATEGY 20180205.pdf

This systematic review and meta-analysis followed the PRISMA (Preferred Reporting Items for Systematic Reviews and Meta-Analyses) guidelines (Moher et al., 2015) and was registered on Prospero (registration ID: CRD42016052062; available at: http://www.crd.york.ac.uk/PROSPERO/display record.php?ID=CRD42016052062). A medical librarian validated the search strategy.

\section{Study Selection}

Three reviewers (N.S., M.K., C.K.) independently assessed all retrieved articles. Overall average pairwise percent agreement between the 3 reviewers was $96.667 \%$ (Fleiss Kappa coefficient $=0.967$; Krippendorff's Alpha $=0$ ). Only one article was disagreed upon by reviewer \#3, and a consensus was reached through discussion between the 3 reviewers.

\section{Data Analysis}

Three authors (N.S., M.K., C.K.) developed a template for data extraction; information extracted included patient characteristics, study design, exercise prescription and 
outcome variables (Kt/v, URR, serum P, QOL, vitamin D3, PTH, CRP, cost effectiveness, hospitalization rate, number of emergency HD, intake of P binders, mortality). Eligible articles were revised, and relevant data was then extracted, and organized into 2 main results tables by one author (N.S.).

The meta-analysis was performed using the Explanatory Software for Confidence Intervals (ESCI) program (Cumming et al., 2012), based on the change in P, QOL-PCS, QOL-MCS and Kt/v which is the difference in mean between the initiation and the end of the intervention. Pooled standard deviation was also calculated using the following formula:

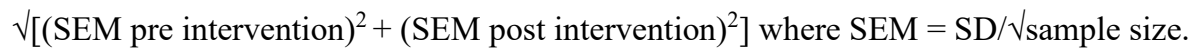
Heterogeneity was assessed across studies using the diamond ratio (DR); a DR $>2$ implies considerable heterogeneity (Jackson \& Turner, 2017). In case of heterogeneity, a randomeffects model was used. Meta-analysis was conducted for outcomes reported in a minimum of 3 studies, as authors suggest greater uncertainty with fewer studies (Cumming \& CalinJageman, 2016). The effect size (ES) of controlled studies was calculated, when possible, to evaluate the effectiveness of the IDE intervention. The reported ES was based on the relative size of Cohen's $d$, which categorizes the studies in the following four levels: $d$ of less than 0.2 was negligible effect, over 0.2 was a small effect, over 0.5 was a medium effect, and over 0.8 was a large effect (Thalheimer $\&$ Cook). The corresponding $95^{\text {th }}$ confidence intervals (CIs) were also reported for each outcome.

\section{RESULTS}

This systematic review was able to identify a wide range of parameters, possibly related to a positive effect of IDE, such as Kt/v, P, QOL, URR, hospitalization, and CRP. After title and abstract screening, the searches resulted in 15092 articles, of which 52 were eligible for full text screening. The authors excluded 25 articles for one of the following reasons: no intervention conducted, study outcomes were not measured, includes children, has mixed types of exercise or non-aerobic exercises, has an intervention duration of $<1$ month, or the full text could not be retrieved even after contacting the authors. Thus 22 articles were included in this review (Figure 1). Only the randomized controlled trials (RCTs) and the interventions with comparison groups with common outcomes were included in the meta-analysis (12 studies). The main characteristics of controlled and uncontrolled studies are presented respectively in Tables 2 and 3. The percentage change of all outcomes reported in table 2 and 3 was calculated by the authors using the pre and post outcome results using 
the following formula: ((Post Outcome Result - Pre Outcome Result)/Pre Outcome Result))*100.

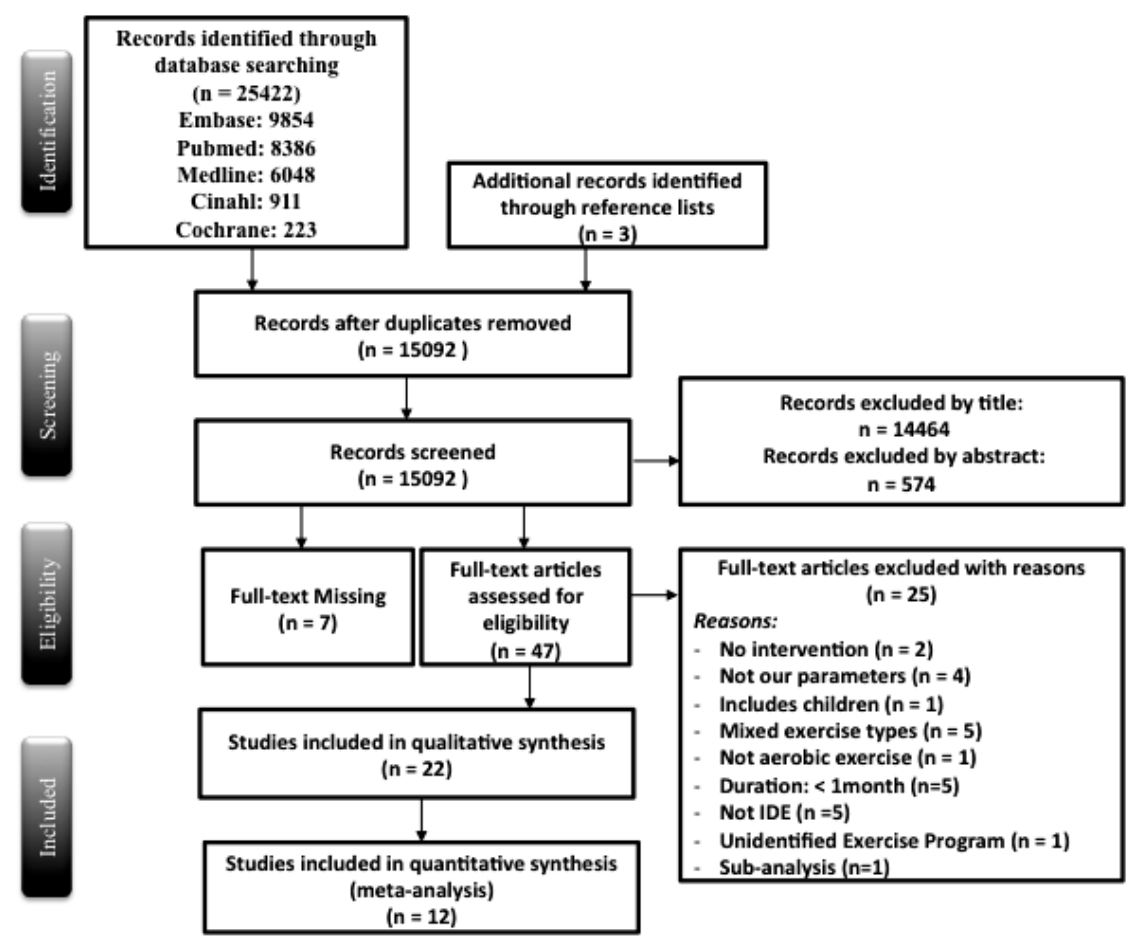

Fig 1 Flow chart of study selection based on the PRISMA statement

\section{General studies' specifications}

The number of participants in a single study ranged from 6-194, and the mean participant's age varied between 43.3-72.5 years. Male gender was predominant in most studies except for 5 (Bae et al., 2015; Dobsak et al., 2012; Painter et al., 2000; Reboredo et al., 2010; Wilund et al., 2010). The IDE in 13 studies consisted of cycling only (Afshar et al., 2010; Besnier et al., 2012; Bohm et al., 2014; de Lima et al., 2013; Giannaki et al., 2013; Groussard et al., 2015; Koh et al., 2010; Musavian et al., 2015; Parker et al., 2015; McMurray et al., 2008; Parsons et al., 2006; Parsons et al., 2004; Wilund et al. 2010), and 8 studies included warm up and stretching in addition to cycling (Bae et al., 2015; Chigira et al., 2017; Dobsak et al., 2012; Golebiowski et al. 2012; Painter et al. 2000; Reboredo et al., 2010; 
Sakkas et al., 2008; Wu et al., 2014). One study consisted of aerobic movement of range capacity only (Makhlough et al., 2012). Exercise intensity was assessed in 11 studies (Afshar et al., 2010; Bae et al., 2015; Bohm et al., 2014; Chigira et al., 2010; de Lima et al., 2013; Koh et al., 2017; Painter et al., 2000; Parker et al., 2015; Reboredo et al., 2010; Wilund et al., 2010; Wu et al., 2014) using the Borg Rating of Perceived Exertion Scale, set between 10 and 16. The other studies used either the patient's maximal exercise capacity, ranging between $30 \%$ and $75 \%$ of oxygen consumption capacity $\left(\mathrm{VO}_{2} \mathrm{Max}\right.$ ) (Dobsak et al., 2012; Giannaki et al., 2013; Groussard et al., 2015; Parsons et al., 2004; Sakkas et al., 2008), or the patient's individual capacity (4 studies) (Golebiowski et al., 2012; Makhlough et al., 2012; McMurray et al., 2008; Parsons et al., 2006). One study did not report the intensity (Musavian et al., 2015) and another one set the cycle speed at 60-70 turns per minute (Besnier et al., 2012). Single IDE session varied in duration between 15-60 minutes where duration of $\geq 30$ minutes was the most used one. As for the length of the interventions, it varied between 2-6 months. IDE was performed on every HD session (2 or 3 times per week).

\section{IDE effects on Quality of Life (QOL)}

A total of 17 studies (Bae et al., 2015; Besnier et al., 2012; Bohm et al., 2014; Chigira et al., 2017; de Lima et al., 2013; Dobsak et al., 2012; Giannaki et al., 2013; Golebiowski et al., 2012; Koh et al., 2010; McMurray et al., 2008; Musavian et al., 2015; Painter et al., 2000; Parsons et al., 2004; Parsons et al., 2006; Reboredo et al., 2010; Sakkas et al., 2008; Wu et al., 2014) showed the effect of aerobic IDE on QOL. Data was very heterogeneous due to the use of different QOL instruments. Accordingly, a meta-analysis including all studies could not be conducted. Table 2 shows detailed data from each study.

A total of 5 studies were included in the meta-analysis (Dobsak et al., 2012; Giannaki et al., 2013; Koh et al., 2010; Painter et al. 2000; Sakkas et al., 2008) and reported on the QOL - physical component scale (PCS) (Table 4) and the QOL - mental component scale (MCS) (Table 4), with a total number of 282 patients (214 patients in the experimental group and 68 patients in the control group). Results showed considerable heterogeneity. Overall, our meta-analysis indicated that aerobic IDE had a significant positive effect on the QOLPCS and on the QOL-MCS. In our meta-analysis, the study with large effect size for the QOL-PCS and the QOL-MCS had the youngest patients in the intervention group and the oldest in the control group (Sakkas et al., 2008). The study with medium effect size for the QOL-PCS was Painter et al. (2000) in which the methodology used was radically different compared to the other studies where they had 2 months of conditioning exercises at home prior to the 2 months of IDE. Moreover, the positive effect of IDE preconditioning on the 
QOL was also seen in the single arm interventional study conducted by Musavian et al. (2015).

As for the total QOL score, the most significant percentual change was reported in an RCT by Wu et al. (2014) (37.7\%) where the design included 5 minutes warm up, and 20 minutes cycling exercise for 3 months at an intensity of 12-16 points on the Borg scale. Also, two single group interventions had high percentual change in the total QOL-SF-36 score: 20.8\% (Musavian et al., 2015) and 14.7\% (Bae et al., 2015) where the latter 2 studies had similar IDE program like Wu et al. (2014) except that they had a longer duration exercise session.

\section{IDE effects on Phosphorus}

Six studies (de Lima et al., 2013; Makhlough et al., 2012; McMurray et al., 2008; Musavian et al., 2015; Reboredo et al., 2010; Wilund et al., 2010) assessed the effect of IDE on serum phosphorus (P) level. Only 3 were eligible to be included in the meta-analysis, showed considerable heterogeneity, and indicated that IDE had no significant effect on serum P levels (Table 4). Makhlough et al. (2012) reported the highest positive effect size and was the only to show significant $P$ decrease (Makhlough et al., 2012); their intervention consisted of aerobic movement of range capacity for 15 minutes per session for 2 months. This study was also unique in the fact of having the highest mean baseline serum P $(7.68 \mathrm{mg} / \mathrm{dl})$ for the IDE group compared to the other studies included in this systematic review. Contrary to the other 2 RCTs (de Lima et al., 2013; Makhlough et al., 2012), Wilund et al. (2010) showed an increase in the P level post IDE; the study had the most prolonged intervention period (4 months) and exercise duration (45 minutes), yet the lowest sample size; the mean starting value for serum $P$ in the intervention group was $5.2 \mathrm{mg} / \mathrm{dl}$.

As for the other 3 single group interventions (McMurray et al., 2008; Musavian et al., 2015; Reboredo et al., 2010), serum P levels were lower at the end of the study, the decrease being statistically significant only in the shortest duration intervention (Musavian et al., 2015), where patients exercised for 30 minutes per session without reporting on intensity. It is worth noting that the 3 studies all started with a P level ranging between $4.82 \mathrm{mg} / \mathrm{dl}$ to 5.7 $\mathrm{mg} / \mathrm{dl}$. 


\section{IDE effects on dialysis efficiency}

Ten studies assessed the effect of IDE on Kt/V (Afshar et al., 2010, Chigira et al., 2017; Dobsak et al., 2012; Groussard et al., 2015; Musavian et al., 2015; Parsons et al., 2004; Parsons et al., 2006; Reboredo et al., 2010; Sakkas et al., 2008) and URR (Dobsak et al., 2012; Musavian et al., 2015; Parker et al., 2015; Parsons et al., 2006). Only 5 were eligible for meta-analysis (Afshar et al., 2010; Dobsak et al., 2012; Groussard et al., 2015; Parsons et al., 2004; Sakkas et al., 2008), and they showed considerable heterogeneity. Their pooled analysis showed that IDE had no significant effect on Kt/V (Table 4). Nevertheless, Dobsak et al. (2012) was able to show a large effect size and a positive change $(+14.6 \%)$ on $\mathrm{Kt} / \mathrm{V}$. This study was unique in its gender distribution (63.7\% female in the intervention group), duration (4.6 months), intensity (30-60\%), and session length (30-50 minutes). Another study conducted by Groussard et al. (2015) had a large effect size with a positive change $(+8.3 \%)$ on $\mathrm{Kt} / \mathrm{v}$. Two other studies also reported a positive change in $\mathrm{Kt} / \mathrm{v}$ post intervention but had a small effect size (Parsons et al., 2004; Sakkas et al., 2008).

In the single intervention group, the 2 studies that resulted in the most relevant change in Kt/v were those by Reboredo et al. (2010) and Parsons et al. (2006); the former study included a 3 months IDE preconditioning before the intervention and the latter one had the longest IDE duration per session and intervention length. Concerning URR, one RCT showed a large effect size and reported a positive change $(+10.9 \%)$ in URR post intervention (Dobsak et al., 2012); the single group intervention study Parson's et al. (2006) also showed a positive URR change and had the longest session length (60 minutes). 


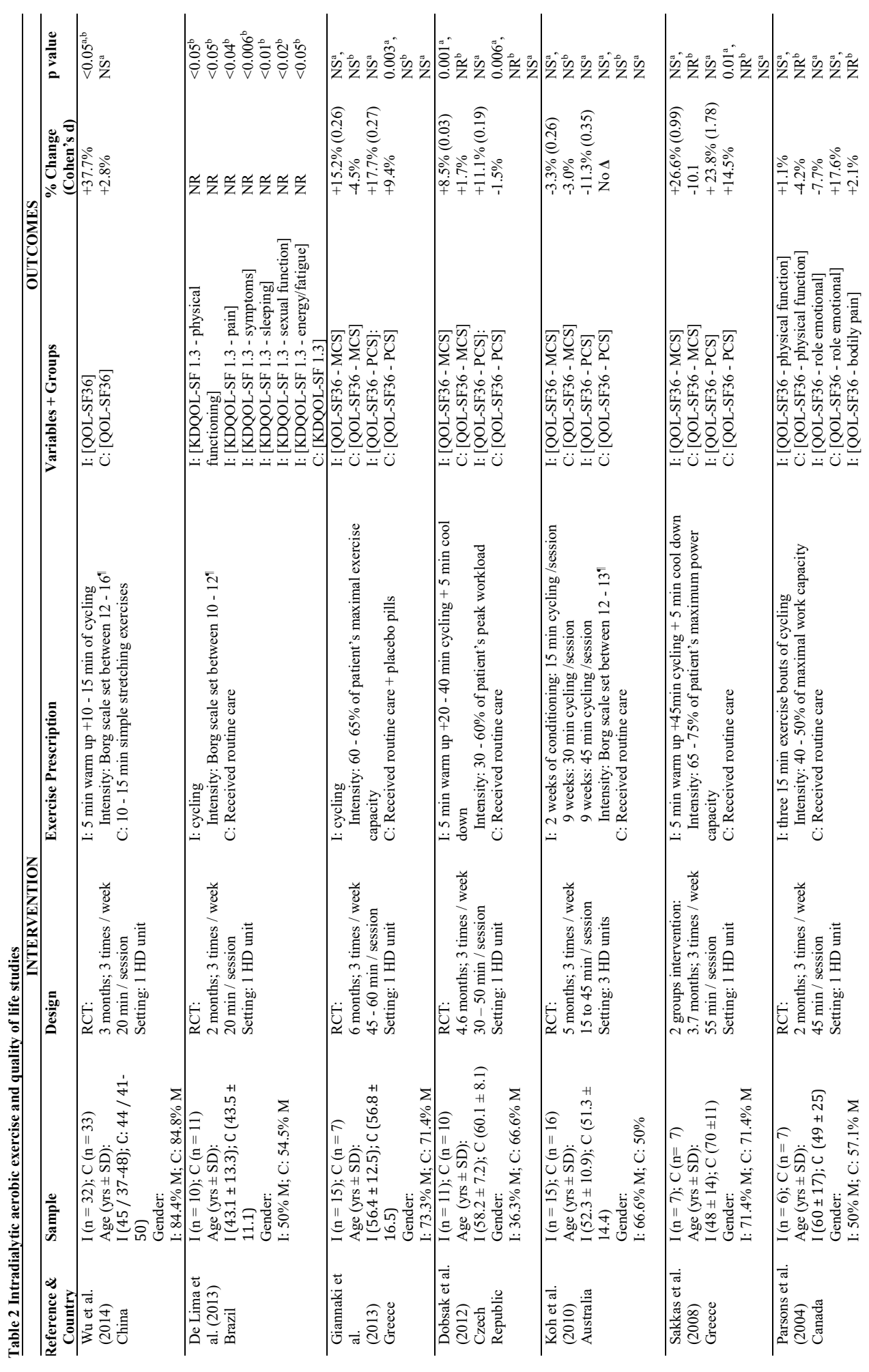




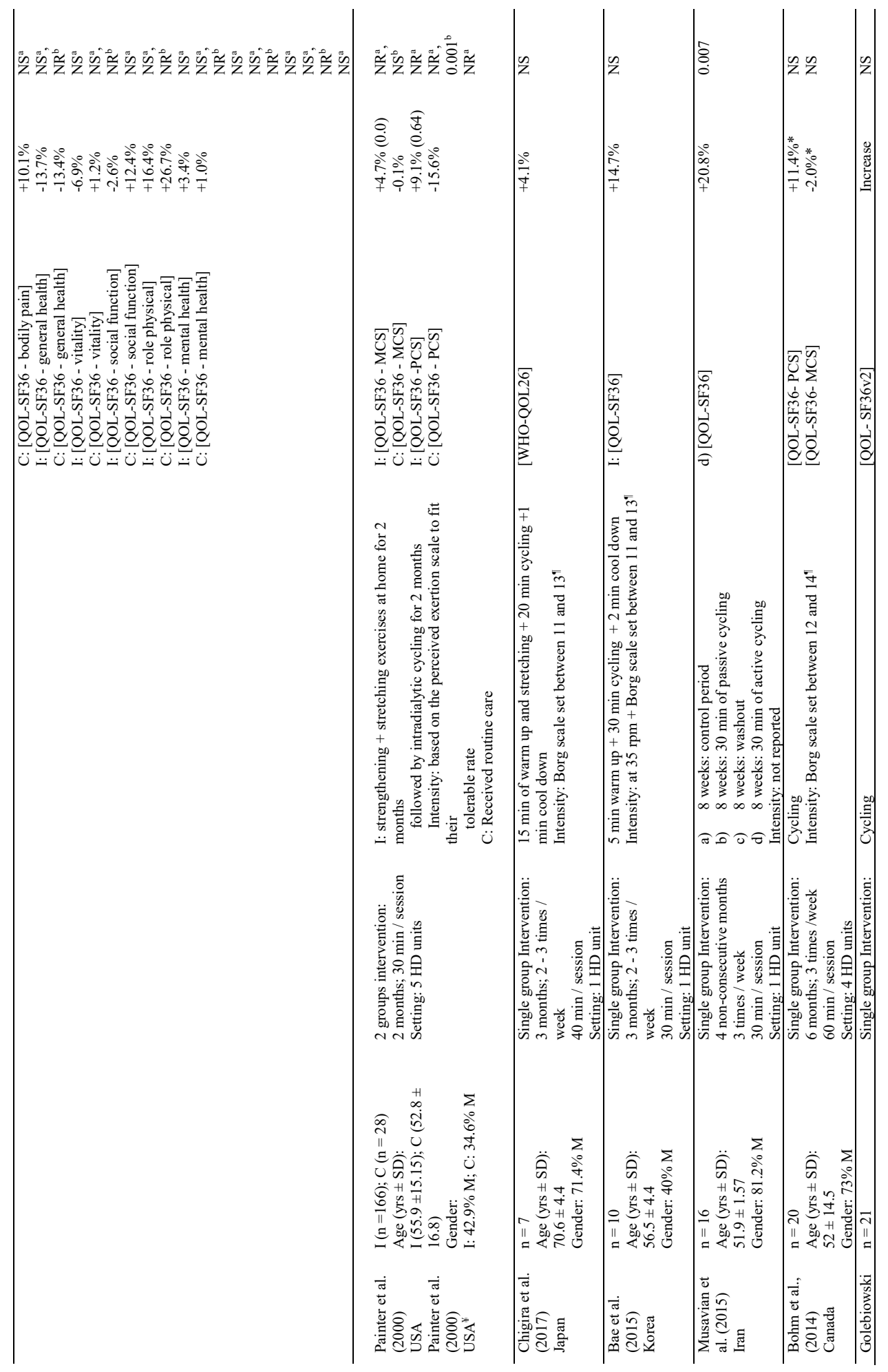




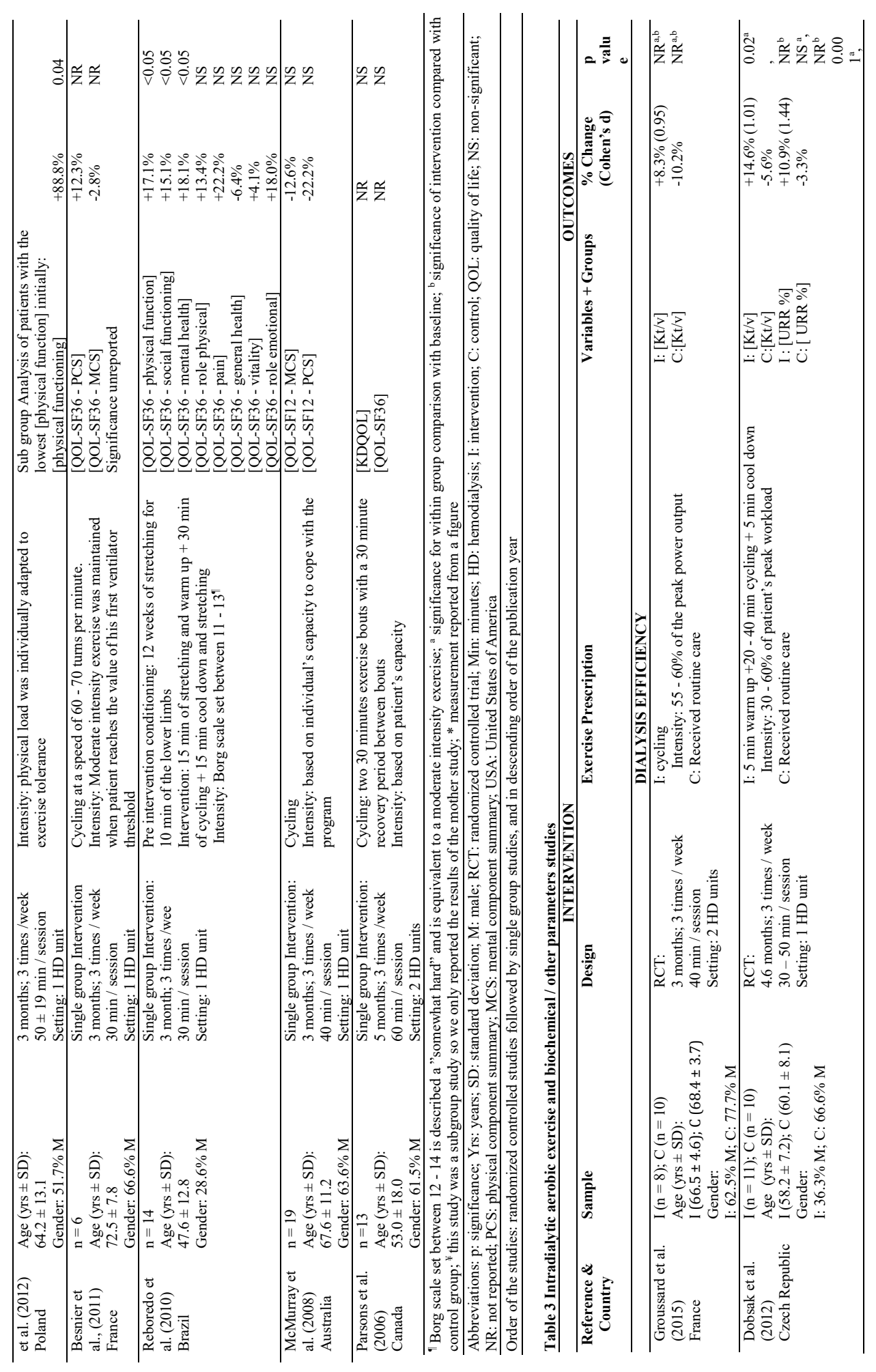




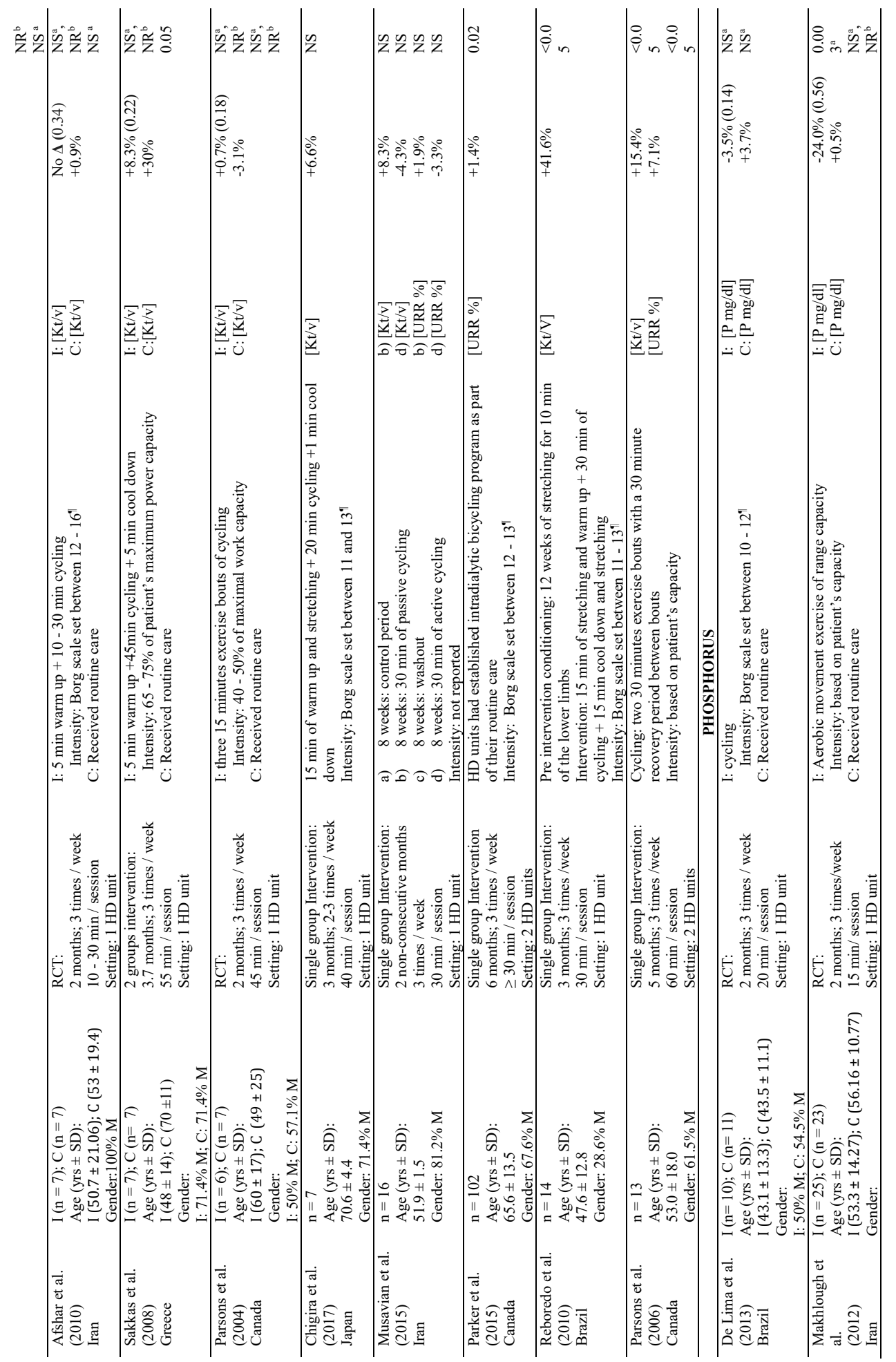




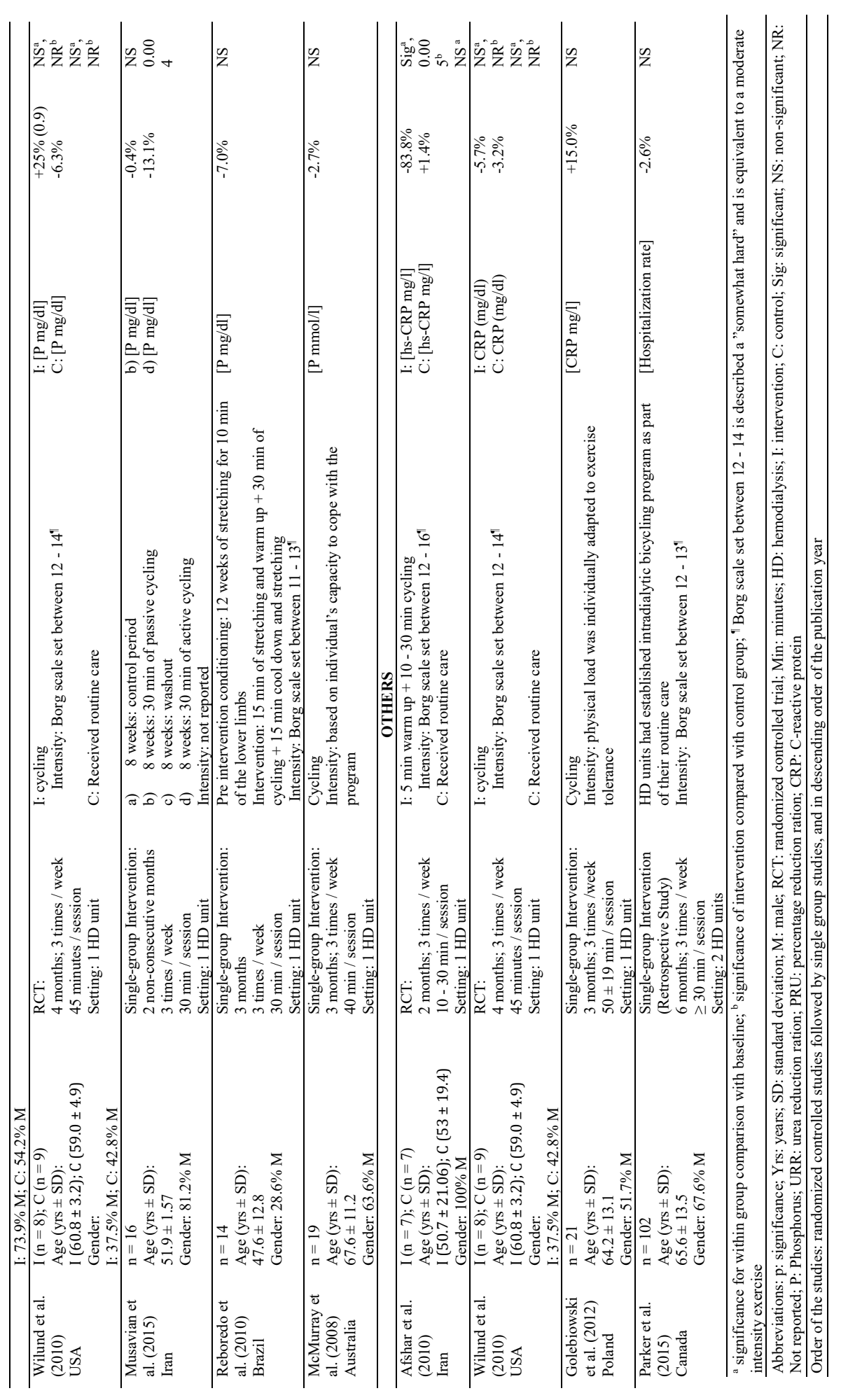




\section{Effect of IDE on inflammatory status variables}

Three studies assessed the effect of IDE on C-reactive protein (CRP) (Afshar et al., 2010; Golebiowski et al., 2012; Wilund et al., 2010). Only 2 were RCTs (Afshar et al., 2010; Wilund et al., 2010) and showed a decrease in CRP level contrary to the single group pilot study (Golebiowski et al., 2012) where the CRP increased but not significantly. Afshar et al. (2010) study showed the most relevant change and was unique in its gender distribution being $100 \%$ males. A meta-analysis was not possible due to the low number of eligible studies reporting on CRP.

\section{Effect of IDE on hospitalization}

Hospitalization rate was reported in only 1 single group intervention (Parker et al., 2015), and showed a non-significant decrease over 6 months.

\section{Effect of IDE on other outcomes}

None of the articles identified through our screening methodology measured vitamin D3, PTH, cost effectiveness, number of emergency HD, intake of P binders, and mortality, which were initially planned to be assessed in this systematic review, thus no reporting was done about these parameters. 


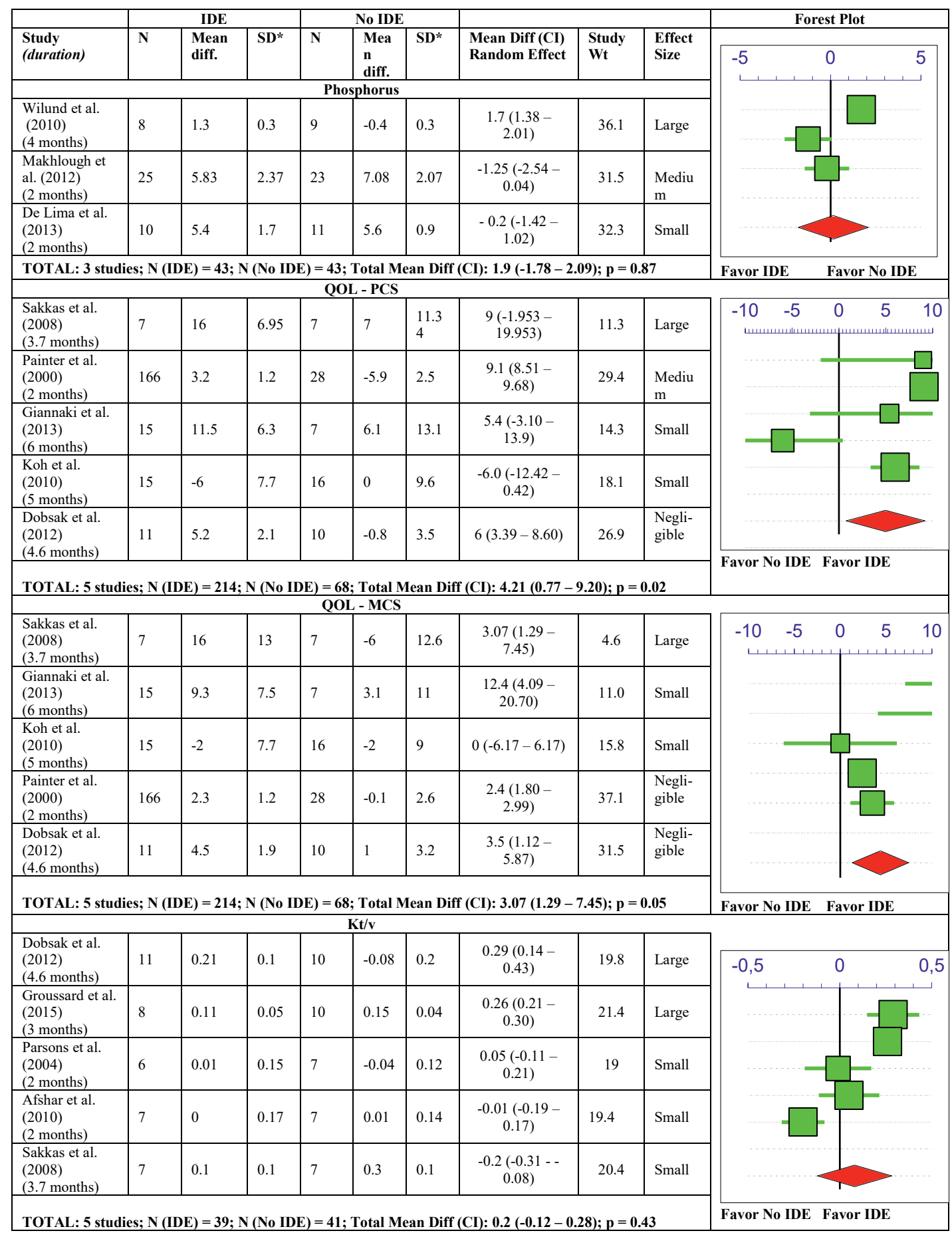




\section{DISCUSSION}

The current document highlighted the effect of aerobic IDE programs in an HD setting. The conclusions derived from this review are not only based on the meta-analysis results, since some of our main outcome parameters were trialed only in studies without comparators.

A meta-analysis of 5 articles showed that aerobic IDE improves the QOL-PCS and the QOL-MCS. This is partly in line with the results reported in the literature, where the meta-analysis of studies on aerobic and resistance IDE programs showed that IDE had a significant difference on the QOL-PCS but not on the QOL-MCS (Sheng et al., 2014; Chung et al., 2017). Pre-IDE muscle conditioning and young age were identified as the key component to the success of patients being able to maintain a long-term IDE program and thus harvest the positive clinical outcomes (Musavian et al., 2015; Painter et al., 2000). However, a recent review conducted by Gomes et al. (2018) showed that aerobic IDE was not associated with improvement in the QOL; in particular, Gomes et al. (2018) meta-analysis included an article where IDE targeted malnourished patients, thus has different selection criteria and its findings cannot be generalized to the general HD population. This current review, however, included 1 article with a large sample size that might have affected the results. In addition, this meta-analysis was based on the outcome change rather than on post intervention values.

This review could not identify an overall effect of IDE on P levels in HD patients. However, a possible effective IDE recipe to improve serum $\mathrm{P}$ was identified by Makhlough et al. (2012) where patients included in the study were hyperphosphatemic (serum $\mathrm{P}>4.5$ $\mathrm{mg} / \mathrm{dl}$ ) [44] and the exercise focused on a 2-month aerobic exercise with range capacity movement. Moreover, Musavian et al. (2015) added the value of preconditioning exercises before implementation of IDE programs on serum P results. These results may be explained by the fact that hyperphosphatemic patients are the ones that need additional therapies to improve their current status, thus they would be the ones that reap the effect of IDE the most (Musavian et al., 2015; Ketteler et al., 2017). Out of the 3 RCT-s focusing on serum P, two of them had an increase in $\mathrm{P}$ in their control groups, thus we postulate that the IDE program reversed the natural deterioration of serum P commonly seen among HD patients; this gives more importance to the positive clinical effect of IDE. HD patients have numerous comorbidities reducing the magnitude of improvement (Painter et al., 2000); this very fact may be more obvious when the parameter taken into account is initially in the normal range. Wilund et al. (2010) had the highest exercise duration and length of intervention, but the $\mathrm{P}$ 
level increased at the end of the intervention; authors did not define the mechanisms of this increase and mentioned their small sample size as a limitation not allowing to control various factors. Besides, Wilund et al. (2010) did not report if dietary P was monitored.

This review did not find an overall positive effect of IDE on Kt/v in HD patients. On the other hand, time seems to be the key for Kt/V improvement by IDE. When comparing the 5 RCTs, characterized by similar duration of exercise per dialysis session and intensity, the most prolonged exercise program (Dobsak et al., 2012) resulted in the most relevant change. Also, gender may play a role since the only intervention that showed no change was in a study on male patients only. In the single group intervention, Reboredo et al. (2010) showed the largest positive significant change $(+41.6 \%)$ in $\mathrm{Kt} / \mathrm{V}$; this study had the youngest age group patients, and the highest female gender percentage among the other comparable studies (Musavian et al., 2015; Parsons et al., 2006; Chigira et al., 2017). It included conditioning before intervention and stretching along with cycling. Another meta-analysis (Sheng et al., 2014) showed that IDE groups had higher Kt/v values than control group; however, the type of exercise included also resistance and stretching (Painter et al., 2000; Mohseni et al., 2013; van Vilsteren et al., 2005), not only aerobic. Unlike Sheng, we were not able to identify a clear effect of aerobic IDE on Kt/V, thus we cannot exclude that other exercise modalities could be more beneficial.

There was no association between IDE and mortality risk, or rates of hospitalization, in the included studies; this was also reported in a previous systematic review (Smart \& Steele, 2011) where no RCT on exercise training with chronic kidney disease patients reported exercise as a direct cause of death. Nephrologists should benefit from this fact to advocate exercise to their patients. Nevertheless, data insufficiency in this field may be a major source of hesitation in the advocacy of IDE.

Other factors beyond those studied in the present paper can provide an argument for IDE. After the initiation of HD therapy, patients' lean tissue mass tends to decrease and fat tissue mass and BMI tend to increase (Marcelli et al., 2016), which may contribute to sarcopenic obesity (Prado et al., 2012). Fluctuation of fat and lean tissue is affected by gender, comorbidities and the initial body composition (Marcelli et al., 2016). Thus, assessing the body composition in HD patients is crucial; especially that BMI is not always a good indicator in this population (Marcelli et al., 2015). Mortality is higher in HD patients with low fat tissue index (FTI) and lean tissue index (LTI); whereas survival is higher in patient with fat and lean tissue compartment lying between the $10^{\text {th }}$ and $90^{\text {th }}$ percentile of a healthy population (Marcelli et al., 2015). It has also been shown that physical inactivity is, in combination with a decline in lean tissue, related to muscle weakness (Souweine et al., 2018), 
which is an important determinant of outcome in dialysis patients (Isoyama et al., 2014).

Whereas resistance-based IDE was shown to increase muscle strength and quadriceps cross sectional area, its effect on LTI was less apparent, this in contrast to treatment with anabolic steroids (Johansen et al. 2006). Therefore, assessing body composition as well as muscle strength on a routine basis can be useful for both risk stratification as well as for targeted interventions.

Cardiovascular diseases are a major comorbidity in HD patients (Longenecker et al. 2002). National and international guidelines for cardiac rehabilitation includes education, exercise training and psychological support (Anderson et al., 2017). HD patients are mostly cardiac patients; therefore, like cardiologist, nephrologists should be encouraged to focus on their patient's behavioral goals by counseling and follow-ups.

HD patients spend an average of 12 hours weekly being sedentary on dialysis; thus it is good opportunity to integrate IDE. IDE might not be a miraculous cure, but it could surely add more functionality to the time spent in HD, improves patient's QOL, decreases anxiety, and maybe increases adherence to the treatment. Perhaps, IDE could be coupled with music, since the music therapy in HD patients showed to reduce anxiety (Kim et al., 2015), pain and nausea (Koca Kutlu \& Eren, 2014), improves blood pressure, quality of sleep, fever, cramps, anxiety and depression levels, pain, and itching (Burrai et al., 2018).

Based on the studies that showed statistically significant positive effects of IDE on all studies parameters in our review, a putative recipe for aerobic exercise in chronic hemodialysis patients could be suggested (Table 5). Exercise, specifically intradialytic, should be part of HD treatment protocol; guidelines are required for IDE to be an adjunct therapy to HD.

Table 5 Putative recipe for aerobic IDE

\begin{tabular}{ll}
\hline Preconditioning & $\begin{array}{l}\text { Strengthening and stretching exercise } 2 \\
\text { months prior to exercise initiation }\end{array}$ \\
Modality & Warm up: Range capacity exercise \\
& + Cycling \\
Frequency & 3 times per week \\
Intensity & Borg scale average rating of 12 points \\
& Or $60-65 \%$ of the peak power output \\
Duration & Range capacity exercises: 15 minutes \\
& + Cycling: $30-45$ minutes \\
\hline
\end{tabular}




\section{Limitations}

The limitations of this review were the lack of quality analysis of the studies. As for the general limitations in the available published literature, they ranged from the selection of the healthier HD patients, to the scarcity of the available interventions, to limited number of eligible interventions for meta-analysis, to the diversity in the exercise intensity, duration and modality. Furthermore, the presence of publication bias in this new researched field cannot be underestimated. The authors could not report on all their primary and secondary outcomes because they were unavailable in the selected studies.

\section{CONCLUSION}

In conclusion, the results of this systematic review suggest that aerobic IDE did not impose any health hazard in HD patients and its incorporation into clinical practice can result in significant improvement in QOL-PCS and QOL-MCS (Dobsak et al., 2012; Giannaki et al., 2013; Koh et al., 2010; Painter et al., 2000; Sakkas et al., 2008).

Since sarcopenic obesity is a prevalent phenomenon among HD patients, this can be a call for making IDE a routine practice in HD units. But the accurate mode of delivery (intensity, type, methods) needs to be tailored to each subgroup of patients within the context of the country, culture, and healthcare system.

Future research should assess the long-term effectiveness and safety of IDE. Most of the studies were conducted in Western countries and thus more insights are needed on other regions, such as the Middle East. Last but not least, mortality rate related to IDE should be studied to strengthen the ground of IDE programs.

Conflict of interest: On behalf of all authors, the corresponding author states that there is no conflict of interest. 


\section{REFERENCES}

Afshar R, Shegarfy L, Shavandi N, Sanavi S (2010) Effects of aerobic exercise and resistance training on lipid profiles and inflammation status in patients on maintenance hemodialysis. Indian J Nephrol, 20(4), 185-189. doi:10.4103/0971-4065.73442

Anderson L, Brown J P, Clark A M, Dalal H, Rossau H K, Bridges C, \& Taylor R S (2017) Patient education in the management of coronary heart disease. Cochrane Database Syst Rev, 6, Cd008895. doi:10.1002/14651858.CD008895.pub3

Bae Y H, Lee S M, Jo J I (2015) Aerobic training during hemodialysis improves body composition, muscle function, physical performance, and quality of life in chronic kidney disease patients. Journal of Physical Therapy Science, 27(5), 1445-1449. doi:10.1589/jpts.27.1445

Besnier F, Laruelle E, Genestier S, Gie S, Vigneau C, Carre F (2012) Effects of exercise training on ergocycle during hemodialysis in patients with end stage renal disease: Relevance of the anaerobic threshold intensity. [French]. [Effets d'un reentrainement a l'effort sur ergocycle pendant les seances de dialyse chez les insuffisants renaux chroniques hemodialyses : interet d'un travail individualise au premier seuil ventilatoire.]. Nephrologie et Therapeutique, 8(4), 231-237. doi:10.1016/j.nephro.2011.10.002

Bohm C, Stewart K, Onyskie-Marcus J, Esliger D, Kriellaars D, Rigatto C (2014) Effects of intradialytic cycling compared with pedometry on physical function in chronic outpatient hemodialysis: a prospective randomized trial. Nephrology Dialysis Transplantation, 29(10), 1947-1955. doi:10.1093/ndt/gfu248

Burrai F, Lupi R, Luppi M, Micheluzzi V, Donati G, Lamanna G et al (2018) Effects of Listening to Live Singing in Patients Undergoing Hemodialysis: A Randomized Controlled Crossover Study. Biol Res Nurs, 1099800418802638. doi:10.1177/1099800418802638

Capitanini A, Lange S, D'Alessandro C, Salotti E, Tavolaro A, Baronti, M E et al. (2014). Dialysis exercise team: the way to sustain exercise programs in hemodialysis patients. Kidney Blood Press Res, 39(2-3), 129-133. doi:10.1159/000355787

Cheema B S, Singh M A (2005) Exercise training in patients receiving maintenance hemodialysis: a systematic review of clinical trials. Am J Nephrol, 25(4), 352-364. doi:10.1159/000087184 
Chigira Y, Oda T, Izumi M, Yoshimura T (2017) Effects of exercise therapy during dialysis for elderly patients undergoing maintenance dialysis. Journal of Physical Therapy Science, 29(1), 20-23. doi:10.1589/jpts.29.20

Chung Y C, Yeh M L, Liu Y M (2017) Effects of intradialytic exercise on the physical function, depression and quality of life for haemodialysis patients: a systematic review and meta-analysis of randomised controlled trials. J Clin Nurs, 26(13-14), 1801-1813. doi:10.1111/jocn.13514

Cumming G, Calin-Jageman R. (2016) Introduction to the new statistics: Estimation, open science, and beyond. Routledge

Cumming G, Fidler F, Kalinowski P, Lai J. (2012) The statistical recommendations of the American Psychological Association Publication Manual: Effect sizes, confidence intervals, and meta-analysis. Australian Journal of Psychology, 64, 138-146.

de Lima M C, Cicotoste Cde L, Cardoso Kda S, Forgiarini L A, Jr Monteiro M B, Dias A S (2013) Effect of exercise performed during hemodialysis: strength versus aerobic. Renal Failure, 35(5), 697-704. doi:10.3109/0886022X.2013.780977

De Moura Reboredo M, Henrique D M N, De Souza Faria R, Chaoubah A, Bastos M G, De Paula R B (2010) Exercise training during hemodialysis reduces blood pressure and increases physical functioning and quality of life. Artificial Organs, 34(7), 586-593. doi: $10.1111 / \mathrm{j} .1525-1594.2009 .00929 . x$

de Souto Barreto P (2017) Exercise for Multimorbid Patients in Primary Care: One Prescription for All? Sports Med, 47(11), 2143-2153. doi:10.1007/s40279-017-0725-Z

Dobsak P, Homolka P, Svojanovsky J, Reichertova A, Soucek M, Novakova M et al. (2012) Intra-dialytic electrostimulation of leg extensors may improve exercise tolerance and quality of life in hemodialyzed patients. Artif Organs, 36(1), 71-78. doi:10.1111/j.1525-1594.2011.01302.x

Giannaki C D, Sakkas G K, Karatzaferi C, Hadjigeorgiou G M, Lavdas E, Kyriakides T et al. (2013) Effect of exercise training and dopamine agonists in patients with uremic restless legs syndrome: a six-month randomized, partially double-blind, placebocontrolled comparative study. BMC Nephrology, 14, 194. doi:10.1186/1471-2369-14194 
Golebiowski T, Kusztal M, Weyde W, Dziubek W, Wozniewski M, Madziarska K et al. (2012) A program of physical rehabilitation during hemodialysis sessions improves the fitness of dialysis patients. Kidney \& Blood Pressure Research, 35(4), 290-296. doi:10.1159/000335411

Gomes Neto M, de Lacerda FFR, Lopes AA, Martinez BP and Saquetto MB (2018) Intradialytic Exercise Training Modalities on Physical Functioning and HealthRelated Quality of Life in Patients Undergoing Maintenance Hemodialysis: Systematic Review and Meta-Analysis. Clinical rehabilitation, 269215518760380, pp. 269215518760380-269215518760380. doi: 10.1177/0269215518760380.

Groussard C, Rouchon-Isnard M, Coutard C, Romain F, Malarde L, Lemoine-Morel S (2015) Beneficial effects of an intradialytic cycling training program in patients with endstage kidney disease. Applied Physiology, Nutrition, \& Metabolism = Physiologie Appliquee, Nutrition et Metabolisme, 40(6), 550-556. doi:10.1139/apnm-2014-0357

Isoyama N, Qureshi A R, Avesani C M, Lindholm B, Barany P, Heimburger O et al. (2014) Comparative associations of muscle mass and muscle strength with mortality in dialysis patients. Clin J Am Soc Nephrol, 9(10), 1720-1728. doi:10.2215/cjn.10261013

Jackson D, Turner R. (2017) Power analysis for random-effects meta-analysis. Research synthesis methods, 2017, 8, 290-302. doi:10.1002/jrsm.1240

Johansen K L, Painter P L, Sakkas G K, Gordon P, Doyle J, \& Shubert T (2006) Effects of resistance exercise training and nandrolone decanoate on body composition and muscle function among patients who receive hemodialysis: A randomized, controlled trial. J Am Soc Nephrol, 17(8), 2307-2314. doi:10.1681/asn.2006010034

Ketteler M, Block GA, Evenepoel P, Fukagawa M, Herzog CA, McCann L et al. (2017) Executive summary of the 2017 KDIGO Chronic Kidney Disease-Mineral and Bone Disorder (CKD-MBD) Guideline Update: what's changed and why it matters. Kidney Int. 2017;92:26-36. (2017). doi:10.1016/j.kint.2017.10.001

Kim Y, Evangelista L S, \& Park Y G (2015) Anxiolytic Effects of Music Interventions in Patients Receiving Incenter Hemodialysis: A Systematic Review and Meta-Analysis. Nephrol Nurs J, 42(4), 339-347; quiz 348.

Koca Kutlu A, \& Eren A G (2014) Effects of music on complications during hemodialysis for chronic renal failure patients. Hemodial Int, 18(4), 777-784. doi:10.1111/hdi.12161 
Koh K P, Fassett R G, Sharman J E, Coombes J S, Williams A D (2010) Effect of intradialytic versus home-based aerobic exercise training on physical function and vascular parameters in hemodialysis patients: a randomized pilot study. Am J Kidney Dis, 55(1), 88-99. doi:10.1053/j.ajkd.2009.09.025

Levin A, Stevens P E (2014). Summary of KDIGO 2012 CKD Guideline: behind the scenes, need for guidance, and a framework for moving forward. Kidney Int, 85(1), 49-61. doi:10.1038/ki.2013.444

Longenecker J C, Coresh J, Powe N R, Levey A S, Fink N E, Martin A et al. (2002) Traditional cardiovascular disease risk factors in dialysis patients compared with the general population: the CHOICE Study. J Am Soc Nephrol, 13(7), 1918-1927.

Maheshwari V, Samavedham L, Rangaiah G P (2011) A regional blood flow model for beta2-microglobulin kinetics and for simulating intra-dialytic exercise effect. Ann Biomed Eng, 39(12), 2879-2890. doi:10.1007/s10439-011-0383-5

Makhlough A, Ilali E, Mohseni R, Shahmohammadi S (2012) Effect of intradialytic aerobic exercise on serum electrolytes levels in hemodialysis patients. Iran J Kidney Dis, 6(2), 119-123.

Marcelli D, Brand K, Ponce P, Milkowski A, Marelli C, Ok E et al. (2016) Longitudinal Changes in Body Composition in Patients After Initiation of Hemodialysis Therapy: Results From an International Cohort. J Ren Nutr, 26(2), 72-80. doi:10.1053/j.jrn.2015.10.001

Marcelli D, Usvyat L A, Kotanko P, Bayh I, Canaud B, Etter M et al. (2015) Body composition and survival in dialysis patients: results from an international cohort study. Clin J Am Soc Nephrol, 10(7), 1192-1200. doi:10.2215/cjn.08550814

McMurray A, Blazey L, Fetherston C (2008) The effect of intradialytic foot pedal exercise on blood pressure phosphate removal efficiency and health related quality of life in haemodialysis patients. Renal Society of Australasia Journal, 4(2), 38-45.

Moher D, Shamseer L, Clarke M, Ghersi D, Liberati A, Petticrew M et al. (2015) Preferred reporting items for systematic review and meta-analysis protocols (PRISMA-P) 2015 statement. Syst Rev, 4, 1. doi:10.1186/2046-4053-4-1

Mohseni R, Emami Zeydi A, Ilali E, Adib-Hajbaghery M, Makhlough A (2013) The effect of intradialytic aerobic exercise on dialysis efficacy in hemodialysis patients: a randomized controlled trial. Oman Med J, 28(5), 345-349. doi:10.5001/omj.2013.99 
Musavian A S, Soleimani A, Masoudi Alavi N, Baseri A, Savari F (2015) Comparing the effects of active and passive intradialytic pedaling exercises on dialysis efficacy, electrolytes, hemoglobin, hematocrit, blood pressure and health-related quality of life. Nurs Midwifery Stud, 4(1), e25922.

O'Hare A M, Tawney K, Bacchetti P, Johansen K L (2003) Decreased survival among sedentary patients undergoing dialysis: results from the dialysis morbidity and mortality study wave 2. Am J Kidney Dis, 41(2), 447-454.

doi:10.1053/ajkd.2003.50055

Painter P, Carlson L, Carey S, Paul S M, Myll J (2000) Physical functioning and healthrelated quality-of-life changes with exercise training in hemodialysis patients. American Journal of Kidney Diseases, 35(3), 482-492.

${ }^{¥}$ Painter P, Carlson L, Carey S, Paul S M, Myll J (2000) Low-functioning hemodialysis patients improve with exercise training. Am J Kidney Dis, 36(3), 600-608. doi:10.1053/ajkd.2000.16200

Parker K, Zhang X, Lewin A, MacRae J M (2015) The association between intradialytic exercise and hospital usage among hemodialysis patients. Applied Physiology, Nutrition, \& Metabolism = Physiologie Appliquee, Nutrition et Metabolisme, 40(4), 371-378. doi:10.1139/apnm-2014-0326

Parsons T L, Toffelmire E B, King-Van Vlack C E (2006) Exercise training during hemodialysis improves dialysis efficacy and physical performance. Archives of Physical Medicine \& Rehabilitation, 87(5), 680-687.

Parsons T L, Toffelmire E B, King-VanVlack C E (2004) The effect of an exercise program during hemodialysis on dialysis efficacy, blood pressure and quality of life in endstage renal disease (ESRD) patients. Clinical Nephrology, 61(4), 261-274.

Prado C M, Wells J C, Smith S R, Stephan B C, \& Siervo M (2012) Sarcopenic obesity: A Critical appraisal of the current evidence. Clin Nutr, 31(5), 583-601. doi:10.1016/j.clnu.2012.06.010

Sakkas G K, Hadjigeorgiou G M, Karatzaferi C, Maridaki M D, Giannaki C D, Mertens P R et al. (2008). Intradialytic aerobic exercise training ameliorates symptoms of restless legs syndrome and improves functional capacity in patients on hemodialysis: a pilot study. Asaio j, 54(2), 185-190. doi:10.1097/MAT.0b013e3181641b07 
Sheng K, Zhang P, Chen L, Cheng J, Wu C, Chen J (2014) Intradialytic exercise in hemodialysis patients: a systematic review and meta-analysis. Am J Nephrol, 40(5), 478-490. doi:10.1159/000368722

Sietsema KE, Amato A, Adler S G, Brass E P (2004) Exercise capacity as a predictor of survival among ambulatory patients with end-stage renal disease. Kidney Int, 65(2), 719-724. doi:10.1111/j.1523-1755.2004.00411.x

Smart N, Steele M (2011) Exercise training in haemodialysis patients: a systematic review and meta-analysis. Nephrology (Carlton), 16(7), 626-632. doi:10.1111/j.14401797.2011.01471.x

Souweine J S, Kuster N, Chenine L, Rodriguez A, Patrier L, Morena M et al. (2018). Physical inactivity and protein energy wasting play independent roles in muscle weakness in maintenance haemodialysis patients. PLoS One, 13(8), e0200061. doi:10.1371/journal.pone.0200061

Tentori F, Blayney M J, Albert J M, Gillespie B W, Kerr P G, Bommer J et al. (2008) Mortality risk for dialysis patients with different levels of serum calcium, phosphorus, and PTH: the Dialysis Outcomes and Practice Patterns Study (DOPPS). Am J Kidney Dis, 52(3), 519-530. doi:10.1053/j.ajkd.2008.03.020

Thalheimer W, Cook S How to calculate effect sizes from published research: a simplified spreadsheet. www.uv.es/ friasnav/TEdatospublicados.xls. Accessed 10 November 2017

van Loon I N, Bots M L, Boereboom F T J, Grooteman M P C, Blankestijn P J, van den Dorpel M A et al. (2017) Quality of life as indicator of poor outcome in hemodialysis: relation with mortality in different age groups. BMC Nephrol, 18(1), 217. doi:10.1186/s12882-017-0621-7

van Vilsteren M C, de Greef M H, Huisman R M (2005) The effects of a low-to-moderate intensity pre-conditioning exercise programme linked with exercise counselling for sedentary haemodialysis patients in The Netherlands: results of a randomized clinical trial. Nephrol Dial Transplant, 20(1), 141-146. doi:10.1093/ndt/gfh560

Wilund K R, Tomayko E J, Wu P T, Ryong Chung H, Vallurupalli S, Lakshminarayanan B, Fernhall B (2010) Intradialytic exercise training reduces oxidative stress and epicardial fat: a pilot study. Nephrol Dial Transplant, 25(8), 2695-2701. doi:10.1093/ndt/gfq106 
World Health Organization (2010) Global recommendations on physical activity for health. Geneva, WHO (p. 58)

Wu Y, He Q, Yin X, He Q, Cao S, Ying G (2014) Effect of individualized exercise during maintenance haemodialysis on exercise capacity and health-related quality of life in patients with uraemia. Journal of International Medical Research, 42(3), 718-727. doi:10.1177/0300060513509037

Young H M, Hudson N, Clarke A L, Dungey M, Feehally J, Burton J O, Smith A C (2015) Patient and Staff Perceptions of Intradialytic Exercise before and after Implementation: A Qualitative Study. PLoS One, 10(6), e0128995. doi:10.1371/journal.pone.0128995 


\section{Supporting information: Search strategy}

\section{PUBMED}

((renal replacement therapy[mesh:noexp] OR renal dialysis[mesh:noexp] OR hemodiafiltration[mesh:noexp] OR renal insufficiency[mesh] OR renal osteodystrophy[mesh:noexp] OR dialysis[mesh] OR artificial kidney[mesh:noexp]) OR (renal insufficien*[tw] OR renal replacement*[tw] OR renal failure*[tw] OR renal osteodystroph*[tw] OR renal necrosis[tw] OR renal necroses[tw] OR renal artificial[tw]) OR (kidney insufficien*[tw] OR kidney replacement*[tw] OR kidney failure*[tw] OR kidney osteodystroph*[tw] OR kidney necrosis[tw] OR kidney necroses[tw] OR kidney artificial[tw]) OR ((dialy*[tw] OR microdialy*[tw] OR haemodialy*[tw] OR hemodialy*[tw] OR intradialy*[tw] OR haemodiafiltration*[tw] OR hemodiafiltration*[tw] OR haemodiafiltration*[tw] OR hemo-diafiltration*[tw])) OR (cardio renal[tw])) AND ((motor activity[mesh:noexp] OR exercise[mesh:noexp] OR walking[mesh:noexp] OR physical exertion[mesh:noexp] OR physical endurance[mesh:noexp] OR exercise tolerance[mesh:noexp] OR physical fitness[mesh:noexp] OR exercise therapy[mesh:noexp] OR sports[mesh:noexp] OR bicycling[mesh:noexp] OR psychomotor performance[mesh:noexp] OR motor skills[mesh:noexp]) OR (exercise*[tw] OR sport*[tw] OR walk*[tw] OR bicycl*[tw] OR pedaler[tw] OR cycling[tw]) OR (muscle training*[tw] OR muscular training*[tw] OR endurance training[tw] OR aerobic training*[tw] OR physical training[tw] OR muscle capacit*[tw] OR muscular capacit*[tw] OR endurance capacit*[tw] OR aerobic capacit*[tw] OR physical capacit*[tw] OR muscle capab*[tw] OR muscular capab*[tw] OR endurance capab*[tw] OR aerobic capab*[tw] OR physical capab*[tw] OR muscle therap*[tw] OR muscular therap*[tw] OR endurance therap*[tw] OR aerobic therap*[tw] OR physical therap*[tw] OR muscle toleran*[tw] OR muscular toleran*[tw] OR endurance toleran*[tw] OR aerobic toleran*[tw] OR physical toleran*[tw] OR muscle prescri*[tw] OR muscular prescri*[tw] OR endurance prescri*[tw] OR aerobic prescri*[tw] OR physical prescri*[tw] OR muscle interven*[tw] OR muscular interven*[tw] OR endurance interven*[tw] OR aerobic interven*[tw] OR physical interven*[tw] OR muscle techni*[tw] OR muscular techni*[tw] OR endurance techni*[tw] OR aerobic techni*[tw] OR physical techni*[tw]) OR (physical therap*[tw] OR physical activi*[tw] OR physical fit[tw] OR physical fitness[tw] OR physical function*[tw] OR physical exert*[tw] OR physical endurance[tw] OR physical modalit*[tw] OR motion therap*[tw] OR motion activi*[tw] OR motion fit[tw] OR motion fitness[tw] OR motion function*[tw] OR motion exert*[tw] OR 
motion endurance[tw] OR motion modalit*[tw] OR physically fit[tw]) OR (motor activit*[tw] OR motor skill*[tw] OR psychomotor performance[tw]))

\section{MEDLINE using OVID}

Database: Ovid MEDLINE(R) Epub Ahead of Print, In-Process \& Other Non-Indexed Citations, Ovid MEDLINE(R) Daily and Ovid MEDLINE(R) $<1946$ to Present> Search Strategy:

1 renal replacement therapy/ or renal dialysis/ or hemodiafiltration/ or hemodialysis, home/

2 exp renal insufficiency/ or renal osteodystrophy/

3 exp Dialysis/

4 Kidneys, Artificial/

5 ((renal or kidney) adj2 (insufficien* or replacement* or failure* or osteopdystroph* or necros?s or artificial)).tw.

6 (dialy* or microdialy* or h? emodialy* or intradialy* or h?emodiafiltration* or h? emo-diafiltration*).tw.

7 (cardio adj renal).tw.

8 or $/ 1-7$

9 motor activity/ or exercise/ or walking/ or physical exertion/ or physical endurance/ or exercise tolerance/ or physical fitness/

10 Exercise Therapy/

11 sports/ or bicycling/

12 psychomotor performance/ or motor skills/

13 (exercise* or sport* or walk* or bicycl* or pedaler or cycling).tw.

14 ((muscle* or muscular or endurance or aerobic* or physical) adj2 (training or capacit* or capab* or therap* or toleran* or prescri* or interven* or techni*)).tw. 
15 ((physical* or motion*) adj2 (therap* or activit* or fit or fitness or function* or exert* or endurance or modalit*)).tw.

16 ((motor adj2 (activit* or skill*)) or (psychomotor adj2 performance)).tw.

17 or $/ 9-16$

$18 \quad 8$ and 17

\section{EMBASE using OVID}

Database: Embase $<1974$ to 2018 Week 24>

Search Strategy:

1 renal replacement therapy/ or hemodiafiltration/ or hemodialysis/

2 exp kidney failure/ or renal osteodystrophy/

3 exp dialysis/

4 artificial kidney/

5 ((renal or kidney) adj2 (insufficien* or replacement* or failure* or osteopdystroph* or necros?s or artificial)).tw.

6 (dialy* or microdialy* or h?emodialy* or intradialy* or h?emodiafiltration* or h?emodiafiltration*).tw.

7 (cardio adj renal).tw.

8 or $/ 1-7$

9 motor activity/

10 exercise/

11 walking/

12 endurance/

13 exercise tolerance/

14 fitness/

15 kinesiotherapy/

16 sport/ or cycling/ 
17 psychomotor performance/

18 motor performance/

19 (exercise* or sport* or walk* or bicycl* or pedaler or cycling).tw.

20 ((muscle* or muscular or endurance or aerobic* or physical) adj2 (training or capacit* or capab* or therap* or toleran* or prescri* or interven* or techni*)).tw.

21 ((physical* or motion*) adj2 (therap* or activit* or fit or fitness or function* or exert* or endurance or modalit*)).tw.

$22(($ motor $\operatorname{adj} 2(\operatorname{activit} *$ or skill*)) or (psychomotor adj2 performance)).tw.

23 or $/ 9-22$

$24 \quad 8$ and 23

25 limit 24 to $\mathrm{yr}=" 2017-2018 "$

\section{COCHRANE}

\#1 MeSH descriptor: [Renal Replacement Therapy] this term only

\#2 MeSH descriptor: [Renal Dialysis] this term only

\#3 MeSH descriptor: [Hemodiafiltration] this term only

\#4 MeSH descriptor: [Hemodialysis, Home] this term only

\#5 MeSH descriptor: [Renal Insufficiency] explode all trees

\#6 MeSH descriptor: [Renal Osteodystrophy] this term only

\#7 MeSH descriptor: [Dialysis] explode all trees

\#8 MeSH descriptor: [Kidneys, Artificial] this term only

\#9 ((renal or kidney) near/2 (insufficien* or replacement* or failure* or osteopdystroph* or necros?s or artificial)) .tw

\#10 (dialy* or microdialy* or haemodialy* or hemodialy* or intradialy* or hemodiafiltration* or haemodiafiltration* or hemo- diafiltration* or haemodiafiltration*) .tw.

\#11 (cardio near renal) .tw.

$\# 12 \# 1$ or $\# 2$ or $\# 3$ or $\# 4$ or $\# 5$ or \#6 or \#7 or \#8 or \#9 or \#10 \#11

\#13 MeSH descriptor: [Motor Activity] this term only 
\#14 MeSH descriptor: [Exercise] this term only

\#15 MeSH descriptor: [Walking] this term only

\#16 MeSH descriptor: [Physical Exertion] this term only

\#17 MeSH descriptor: [Physical Endurance] this term only

\#18 MeSH descriptor: [Exercise Tolerance] this term only

\#19 MeSH descriptor: [Physical Fitness] this term only

\#20 MeSH descriptor: [Exercise Therapy] this term only

\#21 MeSH descriptor: [Sports] this term only

\#22 MeSH descriptor: [Bicycling] this term only

\#23 MeSH descriptor: [Psychomotor Performance] this term only

\#24 MeSH descriptor: [Motor Skills] this term only

\#25 (exercise* or sport* or walk* or bicycl* or pedaler or cycling) .tw

\#26 ((muscle* or muscular or endurance or aerobic* or physical) near/2 (training or capacit* or capab* or therap* or toleran* or prescri* or interven* or techni*)) .tw

\#27 ((physical* or motion*) near/2 (therap* or activit* or fit or fitness or function* or exert* or endurance or modalit*)) .tw.

\#28 ((motor near/2 (activit* or skill*)) or (psychomotor near/2 performance)) .tw

$\# 29 \# 13$ or \#14 or \#15 or \#16 or \#17 or \#18 or \#19 or \#20 or \#21 or \#22 or \#23 or \#24 or $\# 25$ or \#26 or \#27 or \#28

\#30 \#12 and \#29 in Trials

\section{CINAHL using EBSCO}

S1. (MH "Renal Replacement Therapy") OR (MH "Hemodiafiltration") OR (MH

"Hemodialysis") OR (MH "Renal Insufficiency+") OR (MH "Renal Osteodystrophy") OR

(MH "Dialysis+") OR (MH "Kidney, Artificial")

S2. TI (dialysis or microdialysis or hemodialysis or haemodialysis or intradialytic or hemodiafiltration or hemo-diafiltration or haemodiafiltration or haemo-diafiltration)) OR AB 
(dialysis or microdialysis or hemodialysis or haemodialysis or intradialytic or hemodiafiltration or hemo-diafiltration or haemodiafiltration or haemo-diafiltration))

S3. TI ((renal or kidney) W2 (insufficien* or replacement* or failure* or osteopdystroph* or necros\#s or artificial)) OR AB ((renal or kidney) W2 (insufficien* or replacement* or failure* or osteopdystroph* or necros\#s or artificial)) OR MH ((renal or kidney) W2 (insufficien* or replacement* or failure* or osteopdystroph* or necros\#s or artificial))

S4. TI (cardio W renal) OR AB (cardio W renal)

S5. TI (cardio W renal) OR AB (cardio W renal)

S6. (MH "Motor Activity") OR (MH "Exercise") OR (MH "Walking") OR (MH "Exertion") OR (MH "Physical Endurance") OR (MH "Exercise Tolerance") OR (MH "Physical Fitness") OR (MH "Therapeutic Exercise") OR (MH "Sports") OR (MH "Cycling") OR (MH "Motor Skills")

S7. TI ( exercise* or sport* or walk* or bicycl* or pedaler or cycling) OR AB ( exercise* or sport* or walk* or bicycl* or pedaler or cycling)

S8. TI (((muscle* or muscular or endurance or aerobic* or physical) W2 (training or capacit* or capab* or therap* or toleran* or prescri* or interven* or techni*))) OR AB (((muscle* or muscular or endurance or aerobic* or physical) W2 (training or capacit* or capab* or therap* or toleran* or prescri* or interven* or techni*)))

S9. TI (((physical* or motion*) W2 (therap* or activit* or fit or fitness or function* or exert* or endurance or modalit*))) OR AB (((physical or motion*) W2 (therap* or activit* or fit or fitness or function* or exert* or endurance or modalit*)))

S10. TI (((motor) W2 (activit* or skill*)) or (psychomotor W2 performance)) OR AB (((motor) W2 (activit* or skill*)) or (psychomotor W2 performance))

S11. S1 OR S2 OR S3

S12. S6 OR S7 OR S8 OR S9 OR S10

S13. S11 AND S12 

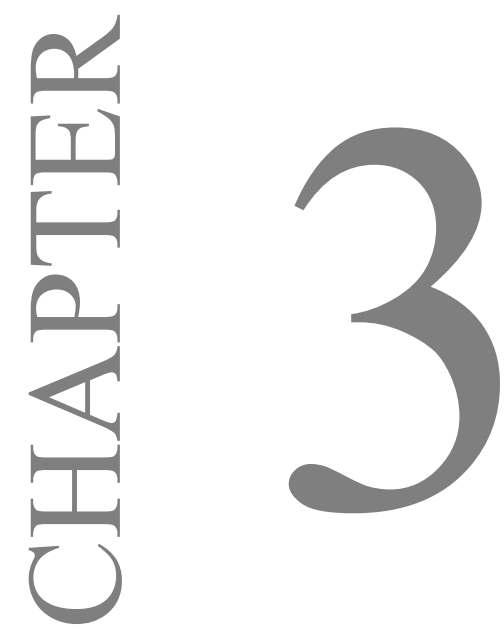

INTRADIALYTIC AEROBIC EXERCISE IN THE UNITED ARAB EMIRATES: A DESCRIPTIVE STUDY

Salhab N, Karavetian M, Kooman J, Fiaccadori E

Arab Journal of Nutrition and Exercise, 2018, 3(1):18-34

[doi:10.8502/ajne.v3i41.1693] 


\begin{abstract}
Aim: Intradialytic exercise (IDE) improves hyperphosphatemia management in hemodialysis (HD) patient in addition to other clinical outcomes. The aim of the study is to present the strategies needed to integrate such a protocol in an HD unit in UAE and patients' baseline characteristics.
\end{abstract}

Methods: The largest HD unit in Sharjah emirate was chosen. All eligible patients $(n=57)$ in the unit were included. Patients were stable adults HD patients who served as their own controls. The intervention included an aerobic low intensity IDE of 45 minutes per HD session, tailored to each patient's fitness scale (BORG scale) for 6 months. Patients were educated on the importance of exercise. Outcome measures were barriers to exercise, serum phosphorus (P), urea reduction ratio (URR), malnutrition inflammation score, quality of life (QOL using euroqol5) collected at baseline and post intervention.

Results: A total of 41 patients completed the study, $61 \%$ were males; $90.2 \%, 53.7 \%$ and $14.6 \%$ suffered from hypertension, diabetes and cardiovascular disease respectively. Hyperphosphatemia was prevalent among $75 \%$ of the patients with a mean of $5.76 \pm 1.66$ $\mathrm{mg} / \mathrm{dl}$. Mean age was $48 \pm 14.37$ years, BMI $24.98 \pm 6.09 \mathrm{~kg} / \mathrm{m}^{2}$, URR $71.88 \pm 8.52 \%$, and $\mathrm{Kt} / \mathrm{v} 1.32 \pm 1.09$. The main barrier to exercise was identified to be fatigue on HD days by $58.5 \%$ of patients, followed by fear of getting hurt (36.6\%). Finally, $80.4 \%$ of patients were mildly malnourished and QOL scale was $65.02 \% \pm 18.54$.

Conclusion: Our study highlighted the widespread of hyperphosphatemia and malnutrition in our sample. The IDE regimen, if proven effective in future studies, could be integrated in the routine practice and may improve patients' outcomes.

Key Words: Hemodialysis, Exercise, Quality of Life, Phosphorus, Urea

Trial registration: www.ClinicalTrials.gov (ID: NCT03131804) 


\section{INTRODUCTION}

Chronic kidney disease (CKD) has been recently recognized as a global health concern (Eckardt et al., 2013) with a prevalence of 13.4\% worldwide (Hill et al., 2016). CKD poses an increased mortality rate compared to their age-matched healthy population (Johansen et al., 2000). In the United Arab Emirates (UAE), the estimated total number of dialysis patients in 2014 is 1870 , out of which $94 \%$ are on hemodialysis, with a prevalence rate of 210 per million population (AlSahow et al., 2016).

Hyperphosphatemia, defined as high serum phosphorus level, is highly prevalent among HD patients and a known risk factor for mortality (Kuhlmann, 2006). The Kidney Disease Improving Global Outcomes (KDIGO) 2017 guidelines recommend lowering the phosphate levels toward the normal range; hyperphosphatemia management includes dialysis, limiting dietary phosphate intake, and the usage of phosphate binder, while restricting the dose of calcium-based phosphate binders (Ketteler et al., 2017). It is documented that a weekly dialysis of more than 30 hours is required for a complete clearance of serum phosphate (P) without the use of P-binders (Kjellstrand et al., 2011); yet, in the UAE, the reality remains that HD sessions in routine practice accumulate to a maximum of 12 hours per week (DOPPS, 2017). Poor serum P control due to high P-to-protein ratio can pose the risk of malnutrition on HD patients, a phenomenon common among these patients (Mathewson et al., 2010). Furthermore, sedentary lifestyle is widespread in HD patients, and stage 4 and 5 CKD cases report to have the lowest physical activity (PA) levels (Zelle et al., 2017). This is despite the fact that the literature is clear on the positive effect of PA on the management of hyperphosphatemia (Musavian et al., 2015), and the negative effect of low PA on quality of life (QOL) (Lopes et al., 2014) and nutritional status (Broers et al., 2015). Broers et al. (2015) was able to demonstrate a positive correlation between the QOL-physical component and nutritional indices. Thus, finding an adjunctive therapy to facilitate hyperphosphatemia management and increase PA levels becomes crucial.

Intradialytic exercise (IDE) has shown to ameliorate solute clearance by increasing blood flow and thus hyperphosphatemia management of HD (Sheng et al., 2014). Patients trained on a cycle ergometer for 20-30 minutes during the first 2 hours of HD increased their phosphate removal (Musavian et al., 2015), and improved their QOL - physical component (de Lima et al., 2013) and their QOL total score (Wu et al. 2014). IDE had a positive effect on dialysis efficiency, specifically on Kt/v (Groussard et. al, 2015) and urea reduction ratio (URR) (Reboredo et. al, 2010). In addition, IDE showed a non-significant decrease in hospitalization rate (Parker et al. 2015). 
For IDE to be a routine procedure for HD patients, an HD unit needs proper exercise equipment, optimal exercise strategies tailored to the patients, trained staff and policies (Parker, 2016). The fact remains that intradialytic training programs are not yet integrated in routine practice worldwide; UAE is not an exception. The feasibility analysis of an IDE program in a developing country is needed, since most of the proof of the efficacy of IDE in HD patients comes from developed western countries.

The aim of the current study is to present the study design and protocol of an aerobic exercise program that will be conducted on HD patients in a selected center in the UAE; another aim is to describe the baseline characteristics of the participants regarding the selected outcome parameters: adequacy of dialysis, serum phosphorus, quality of life, exercise behavior, barriers to exercise and malnutrition inflammation score.

\section{METHODS}

\section{Study Design}

A quasi-experimental intervention with pre and post evaluation design was planned in Al Qassimi hospital's HD unit in Sharjah, UAE. This study is conducted according to the Helsinki Ethical Declaration of 1975 as revised in 1983, and received ethical approved from the Research Ethics Committee of the Ministry of Health and Prevention of UAE (MOHP/DXB/SUBC/NO-5/2016) and that of Zayed University (ZU15_118_F). The study was registered in the ClinicalTrials.gov (ID: NCT03131804). Patients were informed of the study protocol, its risks and benefits, confidentiality, the need for the study team to go through their medical files, and their ability to voluntarily enter and exit the study. The actual patient name list was maintained with the primary investigator (PI), numerical identification was used instead of names to ensure confidentiality and anonymity. Moreover, the filled and signed consent forms and questionnaires were kept with the PI in a locked drawer. 


\section{Study Methodology}

Convenient sample technique was used; all patients in the chosen HD unit, who were eligible, i.e. satisfy the pre-set inclusion criteria, and consented were included in the intervention, resulting in a convenient sampling. Inclusion criteria were stable adult HD patients, on HD for $\geq 3$ months, free of acute diseases and specific cardiovascular problems (cardiac pacemaker, uncontrolled blood pressure, symptomatic ischemic heart disease, arrhythmias, deep vein thrombosis, severe dyspnea), of either gender, not practicing an exercise program at the time of the study, capable to communicate, fully aware of the study protocol, able to perform the cycle pedaler, and provide a consent form. Exclusion criteria were patients not meeting the inclusion criteria; in addition to any of the following: dialyzed from a femoral fistula, suffering from severe anemia (hemoglobin: $<9 \mathrm{~g} / \mathrm{dl}$ ) (Kliger et al., 2013) and/or uncontrolled diabetes.

Five research assistants (RAs) were recruited to conduct the study. They were trained by the PI on study protocol and data collection tools. A licensed physiotherapist of the selected hospital was consulted for the planning of the exercise program, choosing the proper static bicycle and for ongoing supervision of the exercise program.

\section{Sample size}

The outcome is the decrease in serum phosphate. Power analysis was conducted using the GPower 3.1 software (GPower 3.1), based on the effect size $(\mathrm{d}=|0.56|)$ retrieved from Makhlough et al. (2012), an RCT conducted in Iran. The sample size yielded by the power analysis ( $\mathrm{n}=34)$ which was inflated by $72.7 \%$ to account for drop out, leading to a total included sample of 57. We approached all eligible patients $(n=57)$ out of which 41 consented and were included in the analysis.

\section{Conduct of Study}

The study was designed according to 3 phases: baseline ( $\left.T_{0}\right)$, intervention $\left(T_{1}\right)$ and follow-up $\left(\mathrm{T}_{2}\right)$.

Phase 1 (completed): Baseline ( $\left.\mathrm{T}_{0}\right)$ with 2 months duration. The RAs validated the questionnaires, verified the methods that will be used in the intervention on 10 randomly selected patients, and completed the baseline assessment for all consented patients. Furthermore, eligible patients were educated on the importance of exercise, specifically IDE, 
to their health prior to the start of the study by the PI. Finally, patients were assessed using the study outcome measures and data were recorded.

Phase 2 (in progress): Intervention $\left(\mathrm{T}_{1}\right)$ with 6 months duration. The RAs and the physiotherapist will implement the aerobic IDE, using a static cycle pedaler in parallel to an ongoing monthly education on the importance of exercise and on how to integrate it in regular life. The RAs will train the patients on the exercise program and will record the intensity (using the Borg scale) (Centers For Disease Control and Prevention), and duration of the exercise for each patient as well as patient's complaints and reasons for drop out, if any. In addition, the blood pressure and pulse rate will be taken at the start of the exercise and at every 15 minutes onwards. Exercise will not be initiated or will be stopped if the blood pressure is above 200/110 based on recommendation from the literature (de Lima et al., 2013). The exercise program consists of cycling on a static bicycle "Pedal Exerciser KD" for 45 minutes during the first 2 hours of dialysis to avoid stress in the second half of the HD session; the pedaler will be positioned and stabilized in front of the patients while they lied in their dialysis bed. The exercise modality is as follows: a 2 minutes warm up cycling at the lowest resistance of the cycle ergometer (Wong et al., 2017); afterwards, the conditioning phase consists of cycling at patient's preferred resistance, while advising the patient to adjust the intensity to achieve moderate intensity exercise; exercise intensity will be assessed using the Borg scale of perceived exertion and patient will be encouraged to reach an average exertion score of 12. The study will be at all times under the direct supervision of a nephrologist and physiotherapist assigned to the study by the hospital; in case of any discomfort, the intervention will be immediately stopped. As a motivational tool, the patient's activity level (number of minutes exercised) will be recorded on a tracking chart posted in the unit, and the patient with the highest performance will be rewarded at the end of the intervention. At the end of this phase, patients will be evaluated using all the study parameters.

Phase 3: Follow-up ( $\left.\mathrm{T}_{2}\right), 3$ months post intervention. During this phase, there will be no interaction between patients and research team, and at the end, patients will be re-evaluated on all study parameters. 


\section{Data Collection}

Data were collected utilizing validated questionnaires and blood tests from patient hospital charts (Table 1). All questionnaires that were originally not in Arabic (Barriers to Exercise and QOL), were translated to Arabic (local language), prior to the study by 2 translators whose first language was Arabic. Afterwards, each questionnaire was backtranslated to English by a dietitian whose first language was English and who also knew Arabic. The questionnaires will be administered by the recruited RAs at 3 points in time: $T_{0}$, $\mathrm{T}_{1}$ and $\mathrm{T}_{2}$. For quality control purposes, 2 questionnaires will be administered at a time and reviewed prior to administering the next.

\section{Outcome Measures}

1- Objective Tools:

Data from the medical chart of each HD patient were collected, which included: demographics, biochemical markers [Serum Phosphorus (P) mg/dl, Parathyroid Hormone (PTH) pmol/L, Calcium Phosphorus Product (Ca x P) $\mathrm{mg}^{2} / \mathrm{dl}^{2}$, Hemoglobin (HGB) g/dl, Kt/v, Urea Reduction Ratio (URR) \%]. Demographics were collected only at $\mathrm{T}_{0}$ and the remaining information from patient file was collected at $\mathrm{T}_{0}$ (average of 3 months prior to study), and will be collected at $\mathrm{T}_{1}$ (monthly throughout the study), and at $\mathrm{T}_{2}$ (average of 3 months post intervention).

\section{2- Subjective tools}

Four questionnaires are used in this study. All will be administered at 3 phases of the study. RA-s helped patients fill the questionnaires.

- Malnutrition Inflammation Score (MIS) adapted from Kalantar-Zadeh et al. (2004), had 10 components, each with four levels of severity from 0 (normal) to 3 (severely abnormal). The sum of all 10 MIS components ranged from 0 (normal) to 30 (severely abnormal); a higher score reflected severe degree of malnutrition and inflammation.

- Exercise Behavior, attitude and knowledge questionnaire was developed by the authors of this study. It included 7 questions, each with multiple answers to choose from (See Appendix 1, Supplemental Material-1).

-Barriers to physical activity questionnaire adapted from Fiaccadori et al. (2013), included 24 questions that evaluated barriers to physical activity (psychological, physical, and economical barriers) in addition to the lack of time factor. 
- Quality of life will be measured using QOL “EQ-5D-5L” questionnaire (The EuroQol Group, 1990). It assessed 5 health concepts: mobility, self-care, usual activities, pain/discomfort, and anxiety/depression. Each question had 5 answer choices. In addition, patients marked on a visual scale from 0-100 their self-rated health.

\section{Statistical Analysis}

Data were analyzed using the Statistical Package for the Social Sciences (SPSS version 21). Results were considered statistically significant at $\mathrm{P}<0.05$ to guarantee $95 \%$ confidence interval. Descriptive data was conducted, categorical data were reported as frequencies and percentages counts; normality was checked for all continuous variables, and statistical analyses were conducted accordingly (bivariate analysis). In order to keep the tables homogenous, means, SD, min and max were kept on. Moreover, median and interquartile range values were presented for all continuous variables.

\section{RESULTS}

A total of 41 patients met the inclusion criteria and consented out of the 150 patients dialyzing in the selected HD unit (Figure 1).

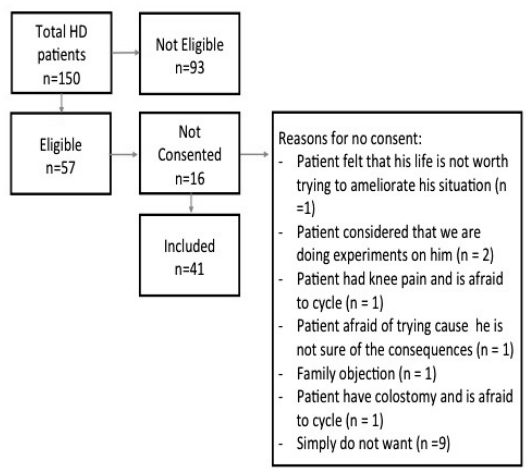

Figure 1 Flow diagram of the patients 


\section{Baseline Sample Characteristics}

A descriptive analysis of baseline data (Tables 1 and 2) showed that $61 \%$ of the population was male with a mean age of 48.0 and mean BMI $24.98 \mathrm{~kg} / \mathrm{m}^{2}$. The majority of the patients were married (78.0\%), and were Arab (Emirati and non Emirati) (75.6\%). More than half of the patients did not work (58.5\%) and $43.9 \%$ had university degrees. Diabetes was the most common primary cause of HD initiation (56.1\%) followed by hypertension (22\%). The most common comorbidities included hypertension (90.2\%) followed by diabetes (53.7\%). Mean of recorded blood parameters were as follows: serum phosphorus (5.76 $\mathrm{mg} / \mathrm{dl})$, calcium and phosphorus byproduct (48.67 $\left.\mathrm{mg}^{2} / \mathrm{dl}^{2}\right)$, PTH (54.28 pmol/L), hemoglobin (10.72 g/dl), Kt/v (1.32), and urea reduction ratio (71.88\%).

Table 1. Baseline Characteristics of Patients (categorical data) $(n=41)$

\begin{tabular}{|c|c|c|c|}
\hline & n (\%) & & n (\%) \\
\hline Gender, Male & $25(61)$ & $\begin{array}{l}\text { Primary Cause of Hemodialysis } \\
\text { Diabetes } \\
\text { Hypertension } \\
\text { Nephritis } \\
\text { Others } \\
\end{array}$ & $\begin{array}{l}23(56.1) \\
9(22) \\
5(12.2) \\
4(9.8)\end{array}$ \\
\hline $\begin{array}{l}\text { Nationality } \\
\text { Emirati } \\
\text { Non-Emirati - Arab } \\
\text { Non-Emirati - Other }\end{array}$ & $\begin{array}{l}8(19.5) \\
23(56.1) \\
10(24.4) \\
\end{array}$ & $\begin{array}{l}\text { Hemodialysis Initiation } \\
<1 \text { year } \\
1-4 \text { years } \\
>4 \text { years }\end{array}$ & $\begin{array}{l}12(29.3) \\
15(36.6) \\
14(34.1)\end{array}$ \\
\hline $\begin{array}{l}\text { Social Status } \\
\text { Single } \\
\text { Married } \\
\text { Other }\end{array}$ & $\begin{array}{l}8(19.5) \\
32(78) \\
1(2.4)\end{array}$ & $\begin{array}{l}\text { Comorbidities* } \\
\text { Diabetes } \\
\text { Hypertension } \\
\text { Cardiovascular Disease } \\
\text { Others }\end{array}$ & $\begin{array}{l}22(53.7) \\
37(90.2) \\
6(14.6) \\
13(31.7)\end{array}$ \\
\hline Work, No & $24(58.5)$ & $\begin{array}{l}\text { Hyperphosphatemia } \\
(\text { Serum } \mathrm{P}>4.6 \mathrm{mg} / \mathrm{dl})\end{array}$ & $31(75)^{¥}$ \\
\hline $\begin{array}{l}\text { Education Level } \\
\text { Illiterate } \\
\text { Read and Write } \\
\text { Elementary } \\
\text { High School } \\
\text { University } \\
\end{array}$ & $\begin{array}{l}4(9.8) \\
4(9.8) \\
5(12.2) \\
10(24.4) \\
18(43.9)\end{array}$ & & \\
\hline
\end{tabular}


Table 2. Baseline Characteristics of Patients (continuous data) $(\mathrm{n}=41)$

\begin{tabular}{|l|c|c|c|c|c|}
\hline & $\begin{array}{c}\text { Normal } \\
\text { Values }\end{array}$ & Mean \pm SD & Min/Max & Median & IQR \\
\hline Age (years) & & $48.00 \pm 14.37$ & $20 / 74$ & 50.00 & $36.50-58.00$ \\
\hline BMI (kg/m $\mathbf{2}^{\mathbf{2}}$ & $20-25$ & $24.98 \pm 6.09$ & $17.06 / 46.14$ & 23.32 & $21.26-26.70$ \\
\hline URR (\%) & $\geq 65$ & $71.88 \pm 8.52$ & $50.79 / 89.62$ & 71.69 & $66.14-77.23$ \\
\hline Kt/v & $1.2-1.4$ & $1.32 \pm 1.09$ & $1.08 / 1.63$ & 1.30 & $1.24-1.37$ \\
\hline P (mg/dl) & $2.5-4.5$ & $5.76 \pm 1.66$ & $1.83 / 9.32$ & 5.61 & $4.82-6.48$ \\
\hline Ca x P (mg $\left./ \mathbf{d d}^{2}\right)$ & $\leq 55$ & $48.67 \pm 14.30$ & $15.44 / 82.86$ & 45.65 & $39.11-56.65$ \\
\hline PTH (pmol/L) & $15.90-31.81$ & $54.28 \pm 45.60$ & $8.90 / 243.2$ & 44.50 & $24.40-69.30$ \\
\hline HGB (g/dl) & $13-17$ & $10.72 \pm 1.44$ & $7.53 / 14.1$ & 10.6 & $9.86-11.56$ \\
\hline MIS & 0 & $7.41 \pm 3.06$ & $3 / 17$ & 7.00 & $5.00-9.00$ \\
\hline
\end{tabular}

SD: Standard Deviation; Min: Minimum; Max: Maximum; BMI: Body Mass Index; URR: Urea Reduction Ratio; P: Phophorus; Ca*P:

Calcium Phosphorus Byproduct; PTH: Parathyroid Hormone; HGB: Hemoglobin; MIS: Malnutrition - Inflammation Score; IQR:

Interquartile range.

MIS Scoring System: The total MIS ranges between 0 and 30: 0: normal; 1-10: mildly malnourished; 11-20: moderately malnourished; 21 30: severely malnourished.

Behavior, attitude and knowledge of HD patients regarding exercise are detailed in Table 3. Around one third of the participants reported at baseline that they did not exercise (31.7\%), and more than half of the sample (58.6\%) intended to start exercising after consulting their physician. The majority of the patients $(92.5 \%)$ perceived exercise as important. Around half of the patient knew that exercise is safe (55\%) and were knowledgeable about its health benefits (43.9\%). 
Table 3. Exercise Behavior, Attitude \& Knowledge Questionnaire

\begin{tabular}{|c|c|c|}
\hline & & n (\%) \\
\hline \multirow{12}{*}{$\begin{array}{l}\text { Exercise } \\
\text { Behavior }\end{array}$} & \multicolumn{2}{|l|}{ Frequency of Exercise per Week } \\
\hline & Don't exercise & $13(31.7)$ \\
\hline & Exercise 1-2 time per week & $1(26.8)$ \\
\hline & Exercise 3 or more times per week & $17(41.5)$ \\
\hline & \multicolumn{2}{|c|}{$\begin{array}{l}\text { Duration of Exercise (among patients who reported exercising once or } \\
\text { more) }\end{array}$} \\
\hline & Less than 30 minutes each time & $9(32.1)$ \\
\hline & $30-40$ minutes per each time & $8(28.6)$ \\
\hline & 40 minutes and more each time & $11(29.3)$ \\
\hline & \multicolumn{2}{|c|}{$\begin{array}{l}\text { Exercise Modality*¥ (among patients who reported exercising once or } \\
\text { more) }\end{array}$} \\
\hline & Walking & $26(96.3)$ \\
\hline & Jogging /Dancing/ Aerobics / Others & $4(14.8)$ \\
\hline & Weight training & $2(7.4)$ \\
\hline \multirow{6}{*}{$\begin{array}{l}\text { Attitude to } \\
\text { Exercise }\end{array}$} & \multicolumn{2}{|l|}{ Intent to Start Exercise } \\
\hline & After consulting the doctor & $24(58.6)$ \\
\hline & Does not know & $17(41.5)$ \\
\hline & \multicolumn{2}{|c|}{ Attitude towards Exercise Importance on Health ${ }^{¥}$} \\
\hline & Important & $37(92.5)$ \\
\hline & Not important & $3(7.5)$ \\
\hline \multirow{6}{*}{$\begin{array}{l}\text { Knowledge on } \\
\text { Exercise }\end{array}$} & \multicolumn{2}{|l|}{ Knowledge about Exercise Safety ${ }^{*}$} \\
\hline & Correct knowledge & $22(55.0)$ \\
\hline & False knowledge & $18(45.0)$ \\
\hline & \multicolumn{2}{|c|}{ Knowledge about the Benefits of Exercise for Hemodialysis Patients* } \\
\hline & Correct knowledge & $18(43.9)$ \\
\hline & No knowledge & $23(56.1)$ \\
\hline
\end{tabular}

*Percentages do not sum up due to multiple possible answers; ¥Valid percentages are reported

Perceived barriers to exercise among the study participants (Table 4), revealed only 1 patient (2.4\%) reported no barriers at all, followed by $14.6 \%$ of them reporting 1 barrier, $36.6 \%$ had $2-4$ barriers, $36.6 \%$ had $5-9$ barriers, $9.8 \%$ had 10 or more barriers. The most frequently reported barriers were fatigue on dialysis days $(58.5 \%)$, fear of getting hurt (36.6\%) and fatigue on non-dialysis days (34.1\%). 
Table 4. Perceived Barriers to Physical Activity

\begin{tabular}{|l|l|}
\hline Barriers & $\mathbf{n}(\mathbf{\%})$ \\
\hline Fatigue on dialysis days & $24(58.5)$ \\
\hline Fear of getting hurt & $15(36.6)$ \\
\hline Fatigue on non-dialysis days & $14(34.1)$ \\
\hline Shortness of breath & $12(29.3)$ \\
\hline Too many medical problems & $11(26.8)$ \\
\hline Chest pain & $11(26.8)$ \\
\hline Feelings of helplessness & $10(24.4)$ \\
\hline Pain on dialysis days & $9(22.0)$ \\
\hline Ulcers on legs and feet & $9(22.0)$ \\
\hline Sadness & $9(22.0)$ \\
\hline Inability to travel & $9(22.0)$ \\
\hline Pain on non-dialysis days & $8(19.5)$ \\
\hline Lack of time on dialysis days & $8(19.5)$ \\
\hline Can't afford to exercise & $8(19.5)$ \\
\hline Lack of safe place for exercise & $6(14.6)$ \\
\hline Physician concern & $5(12.2)$ \\
\hline No exercise partner & $5(12.2)$ \\
\hline I don't want to & $5(12.2)$ \\
\hline Family concern & $5(12.2)$ \\
\hline Lack of time on non-dialysis days & $4(9.8)$ \\
\hline Lack of time because of too many medical \\
\hline pppointments & $4(9.8)$ \\
\hline Feeling too old & $3(7.3)$ \\
\hline Not wanting to be seen doing exercise & $2(4.9)$ \\
\hline
\end{tabular}

The mean of self-rated QOL visual analogue scale was $65.02 \pm 18.54$ (min: 25 - Max: 100). As for the rest of the QOL domains (Table 5), almost half of the patients did not have any problem in walking (48.8\%), and most of them were independent in their routine daily self-care (90\%) and activities (68.3\%). Pain and discomfort were reported by $9.8 \%$ of the patients and anxiety and depression by $7.3 \%$. 
Table 5. EuroQOL 5D-5 L Questionnaire

\begin{tabular}{|l|c|c|c|c|c|}
\hline & $\begin{array}{c}\text { No problem } \\
\mathbf{n}(\%)\end{array}$ & $\begin{array}{c}\text { Slight } \\
\text { problem } \\
\mathbf{n}(\%)\end{array}$ & $\begin{array}{c}\text { Moderate } \\
\text { problem } \\
\mathbf{n}(\%)\end{array}$ & $\begin{array}{c}\text { Severe } \\
\text { problem } \\
\mathbf{n}(\%)\end{array}$ & $\begin{array}{c}\text { Unable / } \\
\text { very severe } \\
\text { n (\%) }\end{array}$ \\
\hline Self-Care & $37(90.2)$ & $1(2.4)$ & $1(2.4)$ & $1(2.4)$ & $1(2.4)^{\mathrm{a}}$ \\
\hline Daily Activities & $28(68.3)$ & $2(4.9)$ & $6(14.6)$ & $2(4.9)$ & $3(7.3)^{\mathrm{a}}$ \\
\hline Anxiety / Depression & $24(58.4)$ & $6(14.6)$ & $5(12.2)$ & $3(7.3)$ & $3(7.3)^{\mathrm{a}}$ \\
\hline Mobility & $20(48.8)$ & $9(22)$ & $4(9.8)$ & $5(12.2)$ & $3(7.3)^{\mathrm{b}}$ \\
\hline Pain / Discomfort & $17(41.5)$ & $10(24.4)$ & $4(9.8)$ & $6(14.6)$ & $4(9.8)^{\mathrm{b}}$ \\
\hline
\end{tabular}

a unable; b very severe

\section{DISCUSSION}

The current study is the first of its kind in UAE to assess the effect of IDE on clinical, behavioral, nutritional and quality of life outcomes among HD patients. We were able to reveal patient characteristics on all study parameters.

While existing studies in the region are limited to their descriptive and cross sectional nature (Al Salmi et al., 2016; Al-Ali et al., 2016), or to their short intervention time (Bayoumi and Wakeep, 2015); the current study is unique in its prospective interventional design implementing the IDE program in UAE.

The study sample was similar to the general HD population in the Gulf countries and UAE in particular in many socio-demographic and clinical aspects. Our ethnic and culturally diversified sample is reflective of the nature of the UAE population (Dubai FAQs).

Moreover, our participants were relatively young and more than half were male. This male predominance in the HD population is a global finding (Hecking et al., 2014) and is consistent with "The Dialysis Outcomes and Practice Patterns Study" (DOPPS) results reported on patients in Gulf countries in general and specifically in the UAE (AlSahow et al., 2016). As for the reasons for initiation of HD, diabetes and hypertension were the most common causes in our sample; a similar finding was reported from DOPPS (AlSahow et al., 2016). Finally, hypertension as a comorbidity was prevalent in more than $90 \%$ of the patients in both our sample and the representative sample of DOPPS UAE (AlSahow et al., 2016). Moreover, more than half of the patients did not work and $43.9 \%$ had university degrees; the former figure is comparable to DOPPS results where $62 \%$ of HD population in the UAE were unemployed (AlSahow et al., 2016), and the latter one is comparable to Dubai Statistic 
Center results where $74.1 \%$ of the unemployed citizens had university degrees (Dubai Statistics Center, 2016). All these factors allow us to generalizing our findings to the general UAE HD population.

Hyperphosphatemia was highly prevalent in our sample at baseline (75\%), a number that was far higher than the 38\% reported in the DOPPS-UAE (Al-Ali et al., 2016), but this can be explained by the fact that the latter results were of data collected in 2013 , using a cutoff point for hypherphosphatemia as $6 \mathrm{mg} / \mathrm{dl}$. The current study followed the KDIGO 2017 guidelines using tight serum $P$ control ( $>4.6 \mathrm{mg} / \mathrm{dl}$ ) (Ketteler et al., 2017). Data from other studies, using $5.5 \mathrm{mg} / \mathrm{dl}$ cut-off point showed around $40 \%$ prevalence of hypherphosphatemia in the region (Karavetian et al., 2014) and worldwide (Port et al., 2006). Given the high clinical (Urena, 2017) and financial burden of hyperphosphatemia (White et al., 2006) and its management through phosphate binders and specifically non calcium phosphate binders (Rizk et al., 2016), finding an effective and inexpensive novel means to manage hyperphosphatemia is a necessity in modern nephrology care (Rizk et al., 2017).

The majority of our sample was mildly malnourished (80.4\%). This is in line with previous literature, where malnutrition was common among HD patients globally (Morais et al., 2005) and regionally (Karavetian et al., 2016). In the previous literature, exercise appeared to improve protein energy wasting (Moraes et al., 2014) and thus malnutrition.

Despite the positive attitude of our sample about the importance of exercise, time allocated for the physical activity was limited, and walking was almost the only form of exercise performed. These practices are similar to those noted in Delgado et al. (2012) (Delgado and Johansen, 2012). This is in line with the sedenterism prevalent among HD patients (Zelle et al., 2017). This might be attributed to the widespread lack of knowledge among these patients or to the fact that exercise is not routinely advocated by the nephrologist within HD patient management (Johansen et al., 2003). To date, there are no clear guidelines in the (KDIGO) or the Kidney Disease Outcomes Quality Initiative (KDOQI) (National Kidney Foundation, 2016) on intradialytic exercise intensity, modality, and duration.

Most of our patients reported the existence of at least 1 barrier, $73 \%$ had more than 2 barriers and the most common barrier was "fatigue on dialysis days"; this was in line with other studies (Fiaccadori et al., 2013; Delgado and Johansen, 2012). The second most prevalent barrier in our sample was "fear of getting hurt", and very few stated "I do not want to" for exercise; these findings were unique for our patients and different from the literature (Fiaccadori et al., 2013; Delgado and Johansen, 2012). Barriers related to physical limitations and fears surrounding exercise ranked higher than logistics-related barriers in our sample. 
The QOL visual analogue scale (VAS) was $65.02 \pm 18.54$, which was slightly higher than VAS results reported from other countries (Zyoud et al., 2016). Also, the percentage of patients reporting no problem across the 5 dimensions of EQ-5D-5L was higher than the score reported in another country (Zyoud et al., 2016). Those differences might be attributed to cultural variances. In fact, while conducting the EuroQOL-5D questionnaire, the research team faced a limitation that was most prevalent in the Visual Analogue Scale question, which raised the question of its cultural appropriateness and its suitability to assess the quality of life in this population. Patients showed strong religious beliefs and interpreted sickness as "fate" and "will of God". When asked to rate their health, many of our patients used the word "Thank God" which was difficult to score. This answer was translated to a high score on the scale. Such impact of religious beliefs influencing the QOL assessment among the chronically ill has been previously reported among other cultures as well (Moraes et al., 2014). Further validation of the instrument in religious oriented cultures is recommended.

\section{CONCLUSION}

Our baseline findings revealed the hyperphosphatemics, and malnourished patients and the barriers impeding them from integrating exercise in their lifestyle. Our results may shed light on this overlooked population, and enlighten other researchers and stakeholders about the current situation of hemodialysis patients in the UAE. Furthermore, it will highlight the strategies needed to tailor-made exercise program for HD patients in the UAE. 


\section{ACKNOWLEDGEMENTS}

The study was funded from Zayed University, Dubai, UAE (Research Grant Activity: R16115).

The project was under the supervision of Dr. Harith Muthana, consultant nephrologist at Al Qassimi Hospital. Dr. Muthana assigned a nephrologist for the daily supervision of the research. The physiotherapy department at Al Qassimi Hospital assigned a physiotherapist to help in the exercise program implementation. The College of Natural and Health Sciences department at Zayed University in Dubai and Sharjah campuses provided students who helped us in data collection. Special thanks to the staff and patients in the participating dialysis unit for facilitating the process.

Funding: This work was supported by Zayed University, Dubai, United Arab Emirates Conflict of interest: None 


\section{REFERENCES}

Al-Ali, F.S., Bieber, B.A., Pisoni, R.L., Ezzat, H., AlGhonaim, M., AlHejaili, F.,...GCCDOPPS 5 Study Group, (2016). Nutritional status and outcomes in hemodialysis patients from the gulf cooperation council countries enrolled in the dialysis outcome and practice patters study phase 5 (2012-2015). Saudi J Kidney Dis Transpl. 27(6 Suppl 1), S31-S41.

AlSahow A, AlRukhaimi M, Wakeel JA, Al-Ghamdi S.M.G., AlGhareeb, S., AlAli, F.,... GCC-DOPPS 5 Study Group, (2016). Demographics and key clinical characteristics of hemodialysis patients from the gulf cooperation council countries enrolled in the dialysis outcomes and practice patters study phase 5 (2012-2015). Saudi J Kidney Dis Transpl. 27(6suppl 1), S12-S23.

Al Salmi, I., Al Rukhaimi, A., AlSahow, A., Shaheen, F.A.M., Al-Ghamdi, S.M.G., AlAli, F.,...GCC-DOPPS 5 Study Group, (2016) et al. Mineral bone mineral and its management among hemodialysis patients in the gulf cooperation council: initial findings from the dialysis outcomes and practice patters study (2012-2015). Saudi J Kidney Dis Transpl. 27(6 Suppl 1), S62-S80.

Bayoumi, M.M., \& Al Wakeep, J.S. (2015). Impacts of exercise program on hemodialysis patients' quality of life and physical fitness. Quality in primary care, 23(4), 192-200.

Broers, N. J., Usvyat, L. A., Kooman, J. P., van der Sande, F. M., Lacson, E., Jr., Kotanko, P., \& Maddux, F. W. (2015). Quality of Life in Dialysis Patients: A Retrospective Cohort Study. Nephron, 130(2), 105-112. doi:10.1159/000430814

Centers for Disease Control and Prevention. Borg Rating of Perceived Exertion Scale. Retrieved from https://www.cdc.gov/physicalactivity/basics/measuring/exertion.htm. Accessed 17 September 2017.

de Lima, M. C., Cicotoste Cde, L., Cardoso Kda, S., Forgiarini, L. A., Jr., Monteiro, M. B., \& Dias, A. S. (2013). Effect of exercise performed during hemodialysis: strength versus aerobic. Ren Fail, 35(5), 697-704. doi:10.3109/0886022x.2013.780977

De Moura Reboredo, M., Henrique, D. M. N., De Souza Faria, R., Chaoubah, A., Bastos, M. G., \& De Paula, R. B. (2010). Exercise training during hemodialysis reduces blood pressure and increases physical functioning and quality of life. Artificial Organs, 34(7), 586-593. doi:10.1111/j.1525-1594.2009.00929.x 
Delgado, C., \& Johansen, K. L. (2012). Barriers to exercise participation among dialysis patients. Nephrol Dial Transplant, 27(3), 1152-1157. doi:10.1093/ndt/gfr404

DOPPS Practice Monitor, Gulf Cooperation Council. Dialysis prescription and dose. March 2017. Retrieved from http://www.dopps.org/DPM/GCC/. Accessed September 13, 2017 .

Dubai FAQs. Population of the UAE. Retrieved from www.dubaifaqs.com/population-ofuae.php. Accessed August 24, 2017.

Dubai Statistics Center, 2016. Percentage Distribution of Unemployed 15 years and over by nationality, sex and educational level - Emirate of Dubai. 2016. Retrieved from https://www.dsc.gov.ae/Report/DSC_LFS_2016_03_03.pdf. Accessed 25 September 2017.

Eckardt, K. U., Coresh, J., Devuyst, O., Johnson, R. J., Kottgen, A., Levey, A. S., \& Levin, A. (2013). Evolving importance of kidney disease: from subspecialty to global health burden. Lancet, 382(9887), 158-169. doi:10.1016/s0140-6736(13)60439-0

EuroQol, G. EuroQol--a new facility for the measurement of health-related quality of life. Health Policy, 16(3), 199-208.

Fiaccadori, E., Sabatino, A., Schito, F., Angella, F., Malagoli, M., Tucci, M., . . Regolisti, G. (2014). Barriers to physical activity in chronic hemodialysis patients: a singlecenter pilot study in an Italian dialysis facility. Kidney Blood Press Res, 39(2-3), 169175. doi: $10.1159 / 000355793$

GPower 3.1. Retrieved from https://www.campbellcollaboration.org/escalc/html/EffectSizeCalculator-SMD1.php. Accessed January 152017.

Groussard, C., Rouchon-Isnard, M., Coutard, C., Romain, F., Malarde, L., Lemoine-Morel, S., . . Boisseau, N. (2015). Beneficial effects of an intradialytic cycling training program in patients with end-stage kidney disease. Applied Physiology, Nutrition, \& Metabolism $=$ Physiologie Appliquee, Nutrition et Metabolisme, 40(6), 550-556. doi:10.1139/apnm-2014-0357

Hecking, M., Bieber, B. A., Ethier, J., Kautzky-Willer, A., Sunder-Plassmann, G., Saemann, M. D., . . . Port, F. K. (2014). Sex-specific differences in hemodialysis prevalence and practices and the male-to-female mortality rate: the Dialysis Outcomes and Practice Patterns Study (DOPPS). PLoS Med, 11(10), e1001750. doi:10.1371/journal.pmed.1001750 
Hill, N. R., Fatoba, S. T., Oke, J. L., Hirst, J. A., O'Callaghan, C. A., Lasserson, D. S., \& Hobbs, F. D. (2016). Global Prevalence of Chronic Kidney Disease - A Systematic Review and Meta-Analysis. PLoS One, 11(7), e0158765. doi:10.1371/journal.pone.0158765

Johansen, K.L., Chertow, G.M., Ng, A.V., Mulligan, K., Carey, S., Schoenfeld, P.Y., \& Kent-Braun, J.A. (2000). Physical activity levels in patients on hemodialysis and healthy sedentary controls. Kidney Int, 57, 2564-2570. doi:10.1046/j.15231755.2000.00116.x

Johansen, K. L., Sakkas, G. K., Doyle, J., Shubert, T., \& Dudley, R. A. (2003). Exercise counseling practices among nephrologists caring for patients on dialysis. Am J Kidney Dis, 41(1), 171-178. doi:10.1053/ajkd.2003.50001

Kalantar-Zadeh, K., Kopple, J. D., Humphreys, M. H., \& Block, G. (2004). Comparing outcome predictability of markers of malnutrition-inflammation complex syndrome in haemodialysis patients. Nephrol Dial Transplant, 19(6), 1507-1519. doi:10.1093/ndt/gfh143

Karavetian, M., Abboud, S., Elzein, H., Haydar, S., \& de Vries, N. (2014). Nutritional education for management of osteodystrophy (NEMO) trial: Design and patient characteristics, Lebanon. Nutr Res Pract, 8(1), 103-111. doi:10.4162/nrp.2014.8.1.103

Karavetian, M., Elzein, H., Rizk, R., Jibai, R., \& de Vries, N. (2016). Nutritional education for management of osteodystrophy: Impact on serum phosphorus, quality of life, and malnutrition. Hemodial Int, 20(3), 432-440. doi:10.1111/hdi.12405

KDOQI Clinical Practice Guideline for Hemodialysis Adequacy: 2015 update. (2015). Am J Kidney Dis, 66(5), 884-930. doi:10.1053/j.ajkd.2015.07.015

Ketteler, M., Block, G. A., Evenepoel, P., Fukagawa, M., Herzog, C. A., McCann, L., . . Leonard, M. B. (2017). Executive summary of the 2017 KDIGO Chronic Kidney Disease-Mineral and Bone Disorder (CKD-MBD) Guideline Update: what's changed and why it matters. Kidney Int, 92(1), 26-36. doi:10.1016/j.kint.2017.04.006

Kjellstrand, C. M., Ing, T. S., Kjellstrand, P. T., Odar-Cederlof, I., \& Lagg, C. R. (2011). Phosphorus dynamics during hemodialysis. Hemodial Int, 15(2), 226-233. doi:10.1111/j.1542-4758.2011.00538.x

Kliger, A. S., Foley, R. N., Goldfarb, D. S., Goldstein, S. L., Johansen, K., Singh, A., \& Szczech, L. (2013). KDOQI US commentary on the 2012 KDIGO Clinical Practice 
Guideline for Anemia in CKD. Am J Kidney Dis, 62(5), 849-859.

doi:10.1053/j.ajkd.2013.06.008

Kuhlmann, M. K. (2006). Management of hyperphosphatemia. Hemodial Int, 10(4), 338-345. doi:10.1111/j.1542-4758.2006.00126.x

Lopes, A. A., Lantz, B., Morgenstern, H., Wang, M., Bieber, B. A., Gillespie, B. W., . . Pisoni, R. L. (2014). Associations of self-reported physical activity types and levels with quality of life, depression symptoms, and mortality in hemodialysis patients: the DOPPS. Clin J Am Soc Nephrol, 9(10), 1702-1712. doi:10.2215/cjn.12371213

Makhlough, A., Ilali, E., Mohseni, R., \& Shahmohammadi, S. (2012). Effect of intradialytic aerobic exercise on serum electrolytes levels in hemodialysis patients. Iran J Kidney Dis, 6(2), 119-123.

Mathewson, A. M., Fouque, D., \& Toft, A. J. (2010). Dietary phosphate assessment in dialysis patients. $J$ Ren Nutr, 20(6), 351-358. doi:10.1053/j.jrn.2010.05.014

Moraes, C., Marinho, S. M., da Nobrega, A. C., de Oliveira Bessa, B., Jacobson, L. V., Stockler-Pinto, M. B., . . Mafra, D. (2014). Resistance exercise: a strategy to attenuate inflammation and protein-energy wasting in hemodialysis patients? Int Urol Nephrol, 46(8), 1655-1662. doi:10.1007/s11255-014-0712-3

Morais, A. A., Silva, M. A., Faintuch, J., Vidigal, E. J., Costa, R. A., Lyrio, D. C., . . Pitanga, K. K. (2005). Correlation of nutritional status and food intake in hemodialysis patients. Clinics (Sao Paulo), 60(3), 185-192. doi:/S180759322005000300002

Musavian, A. S., Soleimani, A., Masoudi Alavi, N., Baseri, A., \& Savari, F. (2015). Comparing the effects of active and passive intradialytic pedaling exercises on dialysis efficacy, electrolytes, hemoglobin, hematocrit, blood pressure and healthrelated quality of life. Nurs Midwifery Stud, 4(1), e25922.

Parker, K. (2016). Intradialytic Exercise is Medicine for Hemodialysis Patients. Curr Sports Med Rep, 15(4), 269-275. doi:10.1249/jsr.0000000000000280

Parker, K., Zhang, X., Lewin, A., \& MacRae, J. M. (2015). The association between intradialytic exercise and hospital usage among hemodialysis patients. Applied Physiology, Nutrition, \& Metabolism = Physiologie Appliquee, Nutrition et Metabolisme, 40(4), 371-378. doi:10.1139/apnm-2014-0326

Port, F. K., Pisoni, R. L., Bommer, J., Locatelli, F., Jadoul, M., Eknoyan, G., . . Y Young, E. W. (2006). Improving outcomes for dialysis patients in the international Dialysis 
Outcomes and Practice Patterns Study. Clin J Am Soc Nephrol, 1(2), 246-255. doi:10.2215/cjn.01050905

Rizk, R., Hiligsmann, M., Karavetian, M., \& Evers, S. M. (2016). Economic evaluations of interventions to manage hyperphosphataemia in adult haemodialysis patients: A systematic review. Nephrology (Carlton), 21(3), 178-187. doi:10.1111/nep.12584

Rizk, R., Karavetian, M., Hiligsmann, M., \& Evers, S. (2017). Effect of stage-based education provided by dedicated dietitians on hyperphosphataemic haemodialysis patients: results from the Nutrition Education for Management of Osteodystrophy randomised controlled trial. J Hum Nutr Diet, 30(5), 554-562. doi:10.1111/jhn.12472

Sheng, K., Zhang, P., Chen, L., Cheng, J., Wu, C., \& Chen, J. (2014). Intradialytic exercise in hemodialysis patients: a systematic review and meta-analysis. Am J Nephrol, 40(5), 478-490. doi:10.1159/000368722

Urena Torres, P. A. (2017). [Strategies aiming to control hyperphosphatemia in chronic kidney disease]. Nephrol Ther, 13 Suppl 1, S95-s101. doi:10.1016/j.nephro.2017.01.002

White, A., Odedina, H.X., Campbell, E., \& Segal, R. (2006). The economic burden of endstage renal disease with hyperphosphatemia. Dis Manage Health Outcomes, 14(2), 99-106.

Wong, J., Davis, P., Patidar, A., Zhang, Y., Vilar, E., Finkelman, M., \& Farrington, K. (2017). The Effect of Intra-Dialytic Exercise on Inflammation and Blood Endotoxin Levels. Blood Purif, 44(1), 51-59. doi:10.1159/000455059

Wu, Y., He, Q., Yin, X., He, Q., Cao, S., \& Ying, G. (2014). Effect of individualized exercise during maintenance haemodialysis on exercise capacity and health-related quality of life in patients with uraemia. Journal of International Medical Research, 42(3), 718727. doi:10.1177/0300060513509037

Zelle, D. M., Klaassen, G., van Adrichem, E., Bakker, S. J., Corpeleijn, E., \& Navis, G. (2017). Physical inactivity: a risk factor and target for intervention in renal care. Nat Rev Nephrol, 13(3), 152-168. doi:10.1038/nrneph.2016.187

Zyoud, S. H., Daraghmeh, D. N., Mezyed, D. O., Khdeir, R. L., Sawafta, M. N., Ayaseh, N. A., ... Al-Jabi, S. W. (2016). Factors affecting quality of life in patients on haemodialysis: a cross-sectional study from Palestine. BMC Nephrol, 17(1), 44. doi:10.1186/s12882-016-0257-z 


\section{APPENDIX 1}

EXERCISE BEHAVIOR QUESTIONNAIRE CIRCLE THE CORRECT ANSWER

SEX

1. Male

2. Female

HOW MANY DAYS PER WEEK DO YOU EXERCISE?

1. I don't exercise

2. 1 time per week

3. 2 times per week

4. 3 times per week

5. More than 3 times per week

HOW MANY HOURS PER DAY DO YOU EXERCISE?

1. Less than 30 minutes per day

2. 30 to 40 minutes per day

3. 50 minutes to 1 hour per day

4. 1 to 2 hours per day

5. More than 2 hours per day

WHAT PHYSICAL ACTIVITIES DO YOU USUALLY DO

TO EXERCISE?

(You can choose more than one answer)

1. Weight training

2. Walking

3. Jogging

4. Aerobics

5. Being part of a sports team

6. Dancing

7. Others.
WHAT IS THE EXTEND OF THE IMPORTANCE OF EXERCISE TO YOUR HEALTH?

1. Extremely important

2. Very important

3. Fairly important

4. Not important at all

WHAT IS THE IMPORTANCE OF DOING EXERCISE FOR HEMODIALYSIS PATIENTS?

1. Improves blood pressure

2. Removal of toxins from the blood

3. Improves blood glucose level

4. Improves blood lipid profile

5. Other DO YOU CONSIDER EXERCISING SAFE FOR HEMODIALYSIS PATIENTS

1. Yes, it is safe

2. No, it is not safe WHEN CAN YOU START EXERCISING?

1. Never

2. I don't know

3. Anytime

4. After consulting the doctor WHAT TYPE OF EXERCISE IS CONSIDERED SAFE?

(You can choose more than one answer)

1. Jogging

2. Swimming

3. High Jump

4. Wall Climbing

5. Weight lifting

6. Biking

7. Pressure training 

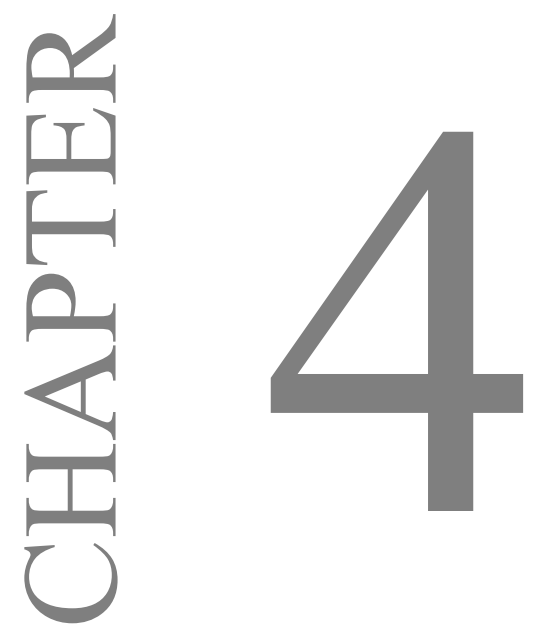

EFFECT OF INTRADIALYTIC AEROBIC EXERCISE ON HYPERPHOSPHATEMIA AND HEALTH OUTCOMES IN HEMODIALYSIS PATIENTS

Salhab N, Alrukhaimi M, Kooman J, Fiaccadori E, Aljubori H, Rizk R, Karavetian $\mathrm{M}$ 


\begin{abstract}
Purpose This study aimed to examine the effect of aerobic intradialytic exercise (IDE) on hyperphosphatemia and other health outcomes among HD patients in the United Arab Emirates (UAE). This was the first trial in the UAE to introduce IDE.
\end{abstract}

Methods Participants were chosen from the largest HD unit in Sharjah emirate for a quasiexperimental intervention with pre and post evaluation. The study lasted for 12 months (2 months familiarization, 6 months intervention, 4 months follow-up). Study parameters were collected at baseline, post intervention and follow-up. The intervention included a moderate intensity aerobic IDE of 45 minutes per HD session; intensity was assessed using the Borg scale. Patients were educated on the importance of exercise. Study outcomes were serum phosphorus (P), pertinent blood tests, urea reduction ratio (URR), malnutrition inflammation score (MIS), and quality of life (QOL) according to EQ-5D-5L.

Results Forty-one eligible consenting HD patients were included in the study. Dropout rate was $25 \%$ post intervention, and $2.4 \%$ at follow-up. Results at follow-up showed a decrease in $\mathrm{P}(\mathrm{p}=0.06)$ in patients who were hyperphosphatemic $(\mathrm{P} \geq 5.5 \mathrm{mg} / \mathrm{dl})$ at baseline, but not in the sample as whole. IDE resulted in a non-significant change in URR $(p=0.47)$ and in the QOL visual analogue scale $(\mathrm{p}=0.34)$. MIS remained stable throughout the study $(\mathrm{p}=0.97)$.

Conclusions In conclusion, aerobic IDE for 45 minutes is safe and could be beneficial, especially for hyperphosphatemic patients. IDE had no consistent effect on QOL. Future powered randomized controlled trials are needed in order to validate this observation.

Trial Registration: ClinicalTrials.gov (ID: NCT03131804)

Keywords: dialysis efficiency, hemodialysis, hyperphosphatemia, intradialytic exercise, quality of life, serum phosphorus 


\section{INTRODUCTION}

Hyperphosphatemia, described as the "silent killer" for hemodialysis (HD) patients (Amann et al. 1999), is prevalent among almost half of this population (Young et al. 2004) and is a determinant to decreased quality of life (QOL) (Chiu et al. 2009). The Kidney Disease Improving Global Outcomes (KDIGO) guidelines (2017) recommend lowering the serum phosphorus (P) levels towards the range that is considered normal for healthy populations. HD alone cannot remove the entire P load; thus, other serum P controlling interventions are utilized such as limiting dietary P intake and administration of P-binding medications (Ketteler et al. 2017). However, these techniques provide some risks: the P-restricted diet can lead to protein energy malnutrition (Ikizler 2009), and P-binders can cause gastrointestinal side effects (St peter et al. 2017), which may increase the cost of health care (White et al. 2006). This is a specific concern cause there are no conclusive evidence about P-binders cost effectiveness as first line intervention for hyperphosphatemia management (Rizk et al. 2016). All of which raises the need for adjunct novel effective approaches targeting hyperphosphatemia management, such as intradialytic exercise (IDE).

IDE has been the focus of many researchers for the last two decades. Orcy et al. (2014) reported an increase in P clearance with IDE among HD patients (Orcy et al. 2014). Also, other studies showed a decrease in serum P levels with IDE (Musavian et al. 2015; Makhlough et al. 2012). Regarding the mechanism behind the effect of IDE on P removal, it is hypothesized that exercise increases blood flow and decreases inter-compartmental resistance leading to increased toxin removal through the dialyzer (Maheshwari et al. 2011). Moreover, IDE potentially has a positive effect on patient's QOL (Musavian et al. 2015; Reboredo et al. 2010; Giannaki et al. 2013; Golebiowski et al. 2012; Wu et al. 2014), dialysis adequacy (Kt/v) (Reboredo et al. 2010 ; Parsons et al. 2006), urea reduction ration (URR) (Parsons et al. 2006; Parker et al. 2015) and C-reactive protein (CRP) levels (Afshar et al. 2010).

Despite the existing literature on the positive effects of IDE, it is not yet a routine practice in HD units. In fact, there are neither set guidelines for HD patients to follow, nor the proper counseling on exercise from the medical team (Regolisti et al. 2018). Accordingly, stronger evidence about the safety, effectiveness and cost-effectiveness of IDE are needed in order to increase the likelihood of implementing it as part of the HD treatment protocol (Sheng et al. 2014; Chung et al. 2017).

The aim of the current study was to assess the effects of a 6-month supervised aerobic IDE program on HD patient's clinical parameters including serum $\mathrm{P}$, parathyroid hormone 
(PTH) levels, dialysis efficiency, nutritional status and QOL. This was the first trial in the UAE to introduce IDE

\section{MATERIALS AND METHODS}

\section{Study Design and Participants}

This was a 6-month, quasi-experimental intervention with pre and post evaluation design. The study protocol was designed in accordance with the Helsinki Ethical Declaration. It was approved by the Research Ethics Committees of the Ministry of Health and Prevention of the UAE (MOHP/DXB/SUBC/NO-5/2016) and Zayed University (ZU15_118_F). The study was prospectively registered in the ClinicalTrials.gov (ID: NCT03131804). Informed consent of all eligible patients was obtained before the initiation of the study. The study protocol and baseline results have been published elsewhere (Salhab et al. 2018).

The study recruited patients attending the HD unit at Al Qassimi hospital, which is the largest HD unit in Sharjah Emirate, United Arab Emirates (UAE), via convenience sampling. The unit nephrologist identified the eligible patients for the study based on the following inclusion criteria: clinically stable, adult, on HD for $\geq 3$ months, free of acute diseases and specific cardiovascular problems (e.g. patients with cardiac pacemaker, or suffering from uncontrolled blood pressure, or symptomatic ischemic heart disease, or arrhythmias, or deep vein thrombosis, or severe dyspnea were not included), not practicing any exercise program at the time of the study, capable to communicate, fully aware of the study protocol, able to perform the cycle pedaler, and willing to provide a consent form. The exclusion criteria for the study were patients not meeting the inclusion criteria, dialyzing from a femoral fistula, suffering from severe anemia (hemoglobin: $<9 \mathrm{~g} / \mathrm{dl}$ ) (Kliger et al. 2013) and/or uncontrolled diabetes. All eligible patients who gave their written informed consent prior to study participation were included in the intervention. The research team consisted of the primary investigator, along with five research assistants; in addition, the hospital assigned a nephrologist and a physiotherapist for the supervision of the IDE program. 


\section{Study protocol}

The study period was 12 months, and was divided into 3 phases (Figure 1), as follows:

1. Baseline or $\mathrm{T}_{0}$ (2 months): This phase comprised the verification of the methods planned for the intervention on 10 randomly chosen patients (results of which were later discarded) This was followed by a one month individualized patient education on the importance of exercise, specifically IDE.

2. Intervention or $T_{1}(6$ months): This phase consisted of an IDE training within the first 2 hours of dialysis, using a static pedaler "Pedal Exerciser KD". The exercise sessions were scheduled for 45 minutes, 2-3 times per week. Before starting the exercise session, the pedaler was positioned and stabilized on the bed in front of the patients while they lied in their dialysis beds. Exercise modality was as follows: patients started their exercise session with a 2 minute warm up, cycling at the lowest resistance of the cycle ergometer (Wong et al. 2017); then they continued cycling at their preferred resistance, and were advised to achieve average exertion score of 12 on the Borg scale of perceived exertion throughout the 6 months of intervention (Center of Disease Control and Prevention). The Borg scale is used to quantify the intensity of exercise and the effort done by patients; Borg scale of 12 - 14 corresponds to a moderate intensity level of exercise (Wong et al. 2017). Exercise intensity, duration, and patient's complaints were recorded for each session. Patient's blood pressure and pulse rate were monitored and recorded at the beginning of the exercise session and at every 15 minutes onwards; exercise was not initiated or was stopped if the blood pressure exceeded $200 \mathrm{mmHg}$ systolic blood pressure and/or $110 \mathrm{mmHg}$ diastolic blood pressure (de Lima e al. 2013) or was below $100 \mathrm{mmHg}$ systolic blood pressure and/or $50 \mathrm{mmHg}$ diastolic blood pressure. Adherence to the IDE was measured as the number of times patients exercised relative to the number of times they were scheduled to exercise. Patient engaging in $\geq 60-80 \%$ of the exercise sessions were considered to have moderate adherence level, and those engaging $>80 \%$ were considered to have high adherence level (Anding et al. 2015). Furthermore, patients were categorized into active versus inactive based on their activity level (based on 20 minutes exercise twice a week for 6 months) (Haskell et al. 2007). The study was at all times under the direct supervision of the medical team; in case of any discomfort, the intervention was immediately stopped. The IDE was done in parallel to an ongoing monthly 20-minute one-to-one patient education on benefits of exercise for HD patients focusing on dialysis efficiency and hyperphosphatemia management, the importance of IDE, how to initiate a safe workout and integrate it in regular routine. Patients were advised to continue exercising at home after the completion of the program. Written 
information was handed to the patient at the end of each educational session. The education material was also kept in the HD unit in the form of a standing poster.

3. Follow-up or $T_{3}$ (4 months): At the end of the intervention, the research team stopped all interaction with the patients, and 3 months post-intervention, assessed them on the study's outcome measures.

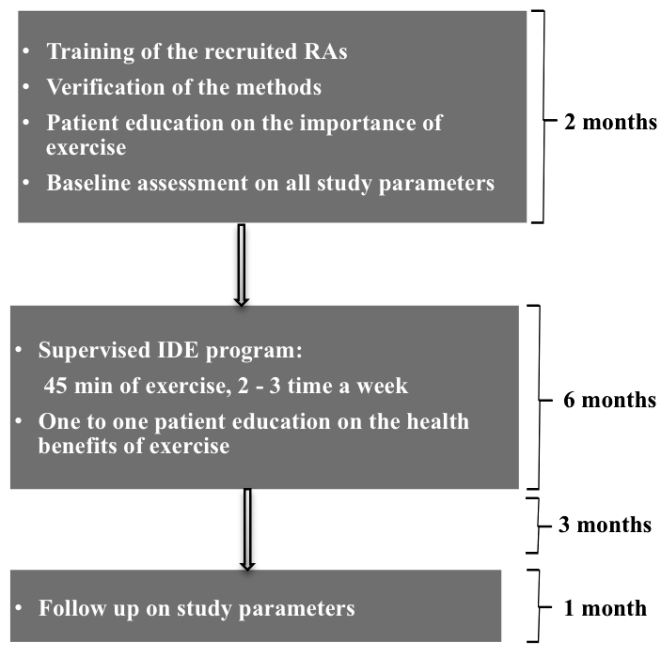

Figure 1. Study Diagram

\section{Outcome Measures}

The primary outcome was serum $\mathrm{P}(\mathrm{mg} / \mathrm{dl})$. The $\mathrm{P}$ change between the active versus inactive patients was analyzed at $T_{0}, T_{1}$, and $T_{2}$, in addition to a sub-analysis among the hyperphosphatemics ( $\mathrm{P} \geq 5.5 \mathrm{mg} / \mathrm{dl}$ ) (Ketteler et al. 2017) compared with the normophasphatemics. Secondary outcomes included PTH (pmol/L), calcium phosphorus byproduct (Ca x P) $\left(\mathrm{mg}^{2} / \mathrm{dl}^{2}\right)$, hemoglobin $(\mathrm{HgB})(\mathrm{g} / \mathrm{dl}), \mathrm{Kt} / \mathrm{v}$, URR (\%), malnutrition inflammation score (MIS) adapted from Kalantar-Zadeh et al. (2004) (Kalantar-Zadeh et al. 2004), and QOL measured using the EQ-5D-5L questionnaire (EuroQol 1990). The MIS questionnaire had 10 components, each with four levels of severity from 0 (normal) to 3 (severely abnormal). The sum of all 10 MIS components ranged from 0 (normal) to 30 (severely malnourished). The EQ-5D-5L questionnaire assessed mobility, self-care, usual activities, pain/discomfort, and anxiety/depression. Each question had 5 answer choices. In addition, patients rated their health on a visual analogue scale (VAS) from 0-100. Adherence 
and reasons for non-adherence to IDE were assessed and recorded by the research team after each session.

\section{Data Collection}

Demographics and blood tests results were collected from the patients' medical files, and questionnaires were conducted by the research team. Blood tests were collected from patients by the hospital staff before the dialysis session, in a non-fasting state. Data collection time-points were at $\mathrm{T}_{0}$ (average of 3 months prior to baseline), $\mathrm{T}_{1}$ (end of the intervention phase) and at $T_{2}$ (average of 3 months post intervention).

\section{Sample Size}

Sample size was estimated on the basis of a power analysis using serum $\mathrm{P}$ as the main outcome variable. It was conducted using the GPower 3.1 software. Assuming a two-sided type I error rate of $5 \%$, a power of $80 \%$ and an effect size of $d=|0.56|$ retrieved from Makhlough et al. (2012), the sample size needed was $n=34$. We approached all eligible patients $(n=57)$ out of whom 41 consented and were included in the analysis. Considering the participation rate and accounting for the drop out rate, the study design was set to be a quasi-experimental intervention with pre and post evaluation without a control group.

\section{Statistical Analysis}

Data were analyzed using the Statistical Package for the Social Sciences (SPSS), version 21 (IBM Corp., Armonk, NY, USA). Results were considered statistically significant at $p \leq 0.05$. Descriptive statistics were used to summarize baseline characteristics of the sample, whereby categorical data were reported as frequencies and percentages counts, and continuous variables were presented as means and standard deviations. Normality was checked for all continuous variables using the Shapiro-Wilk test. Differences between baseline, post intervention and follow-up of normally distributed variables were analyzed by the one-way ANOVA Repeated Measures; those of non-normally distributed data were analyzed by the Friedman Test. We used the pairwise comparison test to compare serum $\mathrm{P}$ in active versus inactive patients based on their activity level defined as $20 \mathrm{~min}$ of exercise twice a week for 6 months (Haskell et al. 2007). 


\section{RESULTS}

Initially, $150 \mathrm{HD}$ patients were assessed for eligibility, 57 were found to be eligible (38\%), and 41 consented to participate ( $28 \%$ refusal rate). Over the course of the intervention phase, 6 patients were not satisfied with the cycle used or the IDE itself (dropped at week 10); one patient was transferred to another HD unit at week 12, 2 patients did a transplant at week 6 and 11, and 1 patient passed away at week 10. At follow-up, 1 patient was transferred to another facility. Thus, 30 patients completed the study (Figure 2).

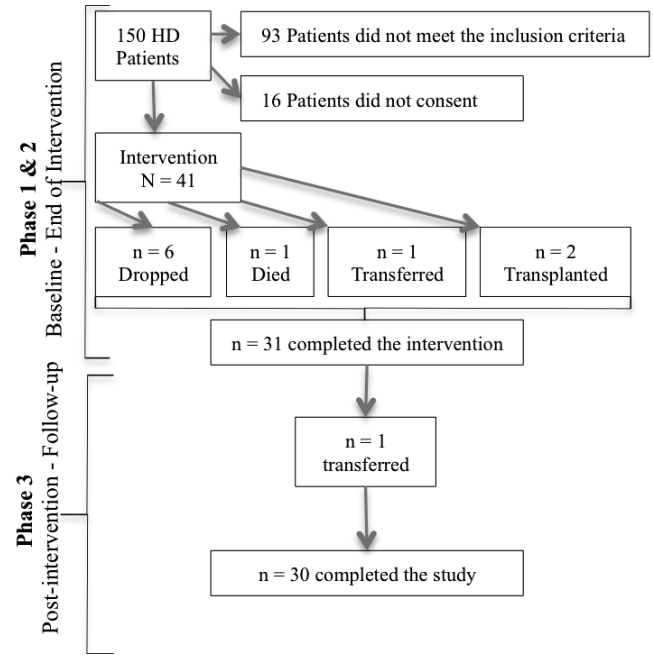

Figure 2. Attrition rate of the study

\section{Characteristics of Patients who completed the Study}

The patients' mean age was $48.87 \pm 11.29$ years, with an equal distribution from both genders, and were mostly Arabs (73.4\%) and Muslims (83.3\%); 96\% of Muslim patients fasted during Ramadan. The primary cause of chronic kidney disease (CKD) was diabetes (53.3\%) followed by hypertension (30\%). The most prevalent comorbidities were hypertension (93.3\%) and diabetes (53.3\%). The mean body mass index (BMI) was $25.25 \pm$ $6.71 \mathrm{~kg} / \mathrm{m}^{2}$. Half of the sample was hyperphosphatemic at baseline. Table 1 illustrates patients' characteristics at baseline for the ones who completed the study; full report on the whole sample at baseline has been published elsewhere (Salhab et al. 2018). 
Table 1. Baseline Characteristics of patients $(n=30)$

\begin{tabular}{|c|c|}
\hline & n (\%) \\
\hline Gender, Male & $15(50)$ \\
\hline \multicolumn{2}{|l|}{ Nationality } \\
\hline Emirati & $5(16.7)$ \\
\hline Non-Emirati - Arab & $17(56.7)$ \\
\hline Non-Emirati - Other & $8(26.7)$ \\
\hline \multicolumn{2}{|l|}{ Primary Cause of CKD } \\
\hline Diabetes & $16(53.3)$ \\
\hline Hypertension & $9(30.0)$ \\
\hline Nephritis & $3(10.0)$ \\
\hline Others & $2(6.7)$ \\
\hline \multicolumn{2}{|l|}{ HD Initiation } \\
\hline$<1$ year & $8(26.7)$ \\
\hline $1-4$ years & $11(36.7)$ \\
\hline$>4$ years & $11(36.7)$ \\
\hline \multicolumn{2}{|l|}{ Comorbidities* } \\
\hline Diabetes & $16(53.3)$ \\
\hline Hypertension & $28(93.3)$ \\
\hline Cardiovascular disease & $4(13.3)$ \\
\hline Others & $8(26.7)$ \\
\hline \multirow[t]{2}{*}{ Phosphorus $\geq 5.5 \mathrm{mg} / \mathrm{dl}$} & $15(50)^{\dagger}$ \\
\hline & Mean \pm SD \\
\hline Age (years) & $48.87 \pm 11.29$ \\
\hline Weight (Kg) & $66.76 \pm 17.59$ \\
\hline $\mathrm{BMI}\left(\mathrm{Kg} / \mathrm{m}^{2}\right)$ & $25.25 \pm 6.71$ \\
\hline${ }^{*}$ Percentages do not sum up due to multiple $\mathrm{p}$ & \\
\hline$\uparrow$ Valid percentages are reported & \\
\hline
\end{tabular}

\section{Aerobic Exercise}

On average, the patients exercised for $140.2 \mathrm{~min} / \mathrm{month}$ (min $111.2 \mathrm{~min}$ - max 166.5 min) on a Borg scale level of 11.3 (min 10.6 - max 11.4) during the 6-months intervention (Figure 3). Monthly patient adherence to the exercise session during the 6 months intervention was as follows: $62 \%$ (Month 1), 48\% (Month 2), 46\% (Month 3), 62\% (Month 4 / Coincides with Ramadan), 64\% (Month 5), 63\% (Month 6). The mean patient adherence rate to the IDE program was $57.5 \%$. Around $26.6 \%$ of the patients were moderately adherent to the program and $20 \%$ of the patients were highly adherent. As for the rest, they had varied levels of adherence, which are detailed in Figure 4. The main reasons behind patients not exercising were feeling tired or simply not wanting to exercise, in addition to problems common to HD patients like leg cramps, leg and back hurting at night after the exercise, burning feet sensation, shortness of breath, fistula or catheter problem, patient feeling down, sleeping during the session or sickness. Only on 5 occasions, the investigators did not initiate exercise because of patient's high blood pressure. 

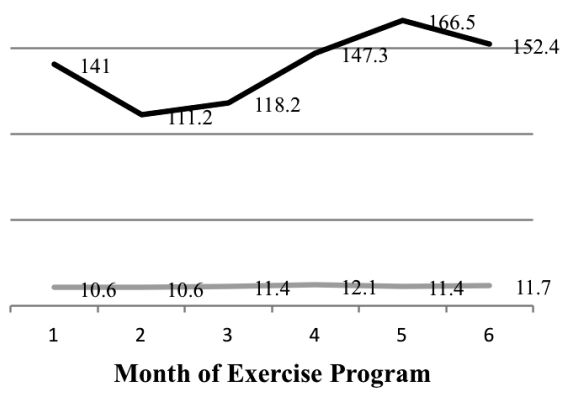

- Average Monthly Borg Scale

Average Monthly Minutes of Exercise

Figure 3. Monthly average minutes of exercise and Borg scale level among patients

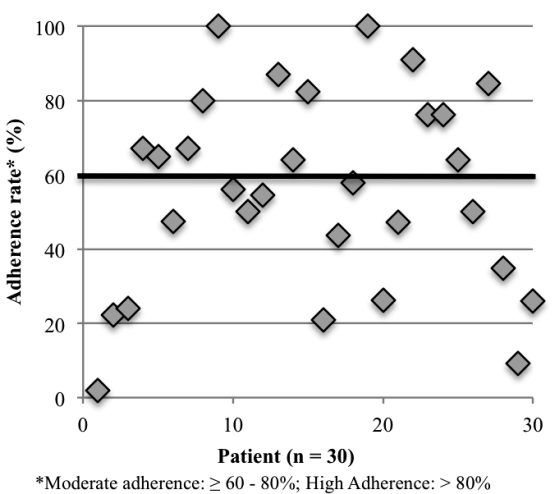

Figure 4. Patients' adherence to the IDE program

\section{Clinical and Laboratory Parameters of Included Patients}

Table 2 shows the laboratory data of patients who completed the study at baseline, after the intervention and at follow-up. There was no significant difference in all studied parameters in the group as a whole. The most change between baseline and follow-up was seen in the MIS (-18.66\%) 
The sub- analysis of active versus inactive patients showed that the mean serum $\mathrm{P}$ changed from $5.64 \pm 1.42$ to $5.11 \pm 1.21 \mathrm{mg} / \mathrm{dl}$ from baseline to follow-up among active patients and from $5.89 \pm 2.1$ to $5.83 \pm 1.79 \mathrm{mg} / \mathrm{dl}$ among inactive patient; this difference was not significant in both groups.

Table 2. Study outcomes of all patients $(n=30)$

\begin{tabular}{lcccc}
\hline & $\begin{array}{c}\text { Mean } \pm \mathrm{SD} \\
\text { Baseline }\end{array}$ & $\begin{array}{c}\text { Mean } \pm \mathrm{SD} \\
\text { Post Intervention }\end{array}$ & $\begin{array}{c}\text { Mean } \pm \mathrm{SD} \\
\text { Follow-up }\end{array}$ & $\begin{array}{c}\mathrm{p} \text {-value } / \\
\% \Delta\end{array}$ \\
\hline $\mathrm{P}(\mathrm{mg} / \mathrm{dl})$ & $5.78 \pm 1.81$ & $5.53 \pm 2.14$ & $5.52 \pm 1.58$ & $0.56 /-4.50$ \\
$\mathrm{PTH}(\mathrm{pmol} / \mathrm{L})$ & $49.15 \pm 31.65$ & $54.58 \pm 38.59$ & $48.80 \pm 35.61$ & $0.52 /-0.71$ \\
$\mathrm{Ca} \mathrm{P}\left(\mathrm{mg}^{2} / \mathrm{dl}^{2}\right)$ & $48.96 \pm 15.68$ & $46.48 \pm 16.48$ & $47.16 \pm 14.07$ & $0.54 /-3.68$ \\
$\mathrm{URR}(\%)$ & $72.93 \pm 9.26$ & $73.68 \pm 12.38$ & $74.38 \pm 10.64$ & $0.47 / 1.99$ \\
$\mathrm{Kt} / \mathrm{V}$ & $1.31 \pm 0.1$ & $1.29 \pm 0.09$ & $1.28 \pm 0.06$ & $0.17 /-2.30$ \\
$\mathrm{HGB}(\mathrm{g} / \mathrm{dl})$ & $10.74 \pm 1.45$ & $11.1 \pm 1.66$ & $10.82 \pm 1.59$ & $0.52 / 0.74$ \\
$\mathrm{MIS}$ & $8.2 \pm 3.46$ & $7.47 \pm 3.39$ & $6.67 \pm 1.98$ & $0.16 /-18.66$ \\
QOL - VAS & $63.03 \pm 17.65$ & $65.67 \pm 19.64$ & $68.66 \pm 17.71$ & $0.34 / 8.93$ \\
\hline
\end{tabular}

Abbreviations: \% $\Delta$ : percentage change from baseline to follow-up; P: phosphorus; PTH: parathyroid hormone; Ca x P: calcium phosphorus byproduct; URR: urea reduction ration; Kt/V: dialysis adequacy; HGB: hemoglobin; MIS: malnutrition inflammation score; QOL - VAS: quality of life - visual analogue scale

Outcome of Patients who were Hyperphosphatemic at Baseline

An additional analysis was done to compare the changes in study parameters between hyperphosphatemics versus normophophatemics. In hyperphosphatemic patients, the serum $\mathrm{P}$ mean \pm SD dropped from $7.04 \pm 1.39$ at baseline to $6.08 \pm 1.68$ at follow-up with a p-value that approached significance $(\mathrm{p}=0.06)$. The prevalence of hyperphosphatemia was $50 \%$ at baseline, $46.6 \%$ post intervention, and $46.6 \%$ at follow-up. There was no significant difference in the rest of the studied parameters between these 2 subgroups.

\section{Quality Of Life (EQ-5D-5L)}

There was no statistically significant difference in the QOL-VAS ( $\mathrm{p}=0.34)$; the percentage change between baseline and follow-up in QOL-VAS was $8.93 \%$. The number of patients having "no problem" in most of EQ-5D-5L domains (self-care, daily activities, mobility) increased post-intervention (Table 3). 
Table 3. EuroQOL 5D-5L Questionnaire $(n=30)$

\begin{tabular}{lllcc}
\hline & No problem & $\begin{array}{c}\text { Slight - } \\
\text { Moderate } \\
\text { problem }\end{array}$ & $\begin{array}{c}\text { Severe } \\
\text { Problem } \\
\text { - Unable }\end{array}$ \\
\hline EQ- dimension & & $\mathrm{n}(\%)$ & $\mathrm{n}(\%)$ & $\mathrm{n}(\%)$ \\
\hline Self-Care & Baseline & $26(86.6)$ & $2(6.7)$ & $2(6.7)$ \\
& Post Intervention & $28(93.3)$ & $2(6.7)$ & $0(0.0)$ \\
& Follow-up & $13(43.3)$ & $13(43.4)$ & $4(13.3)$ \\
\hline Daily & Baseline & $18(60.0)$ & $7(23.3)$ & $5(16.7)$ \\
Activities & Post Intervention & $24(80.0)$ & $6(20.0)$ & $0(0.0)$ \\
& Follow-up & $24(80.0)$ & $5(16.7)$ & $1(3.3)$ \\
\hline Anxiety / & Baseline & $18(60.0)$ & $7(23.3)$ & $5(16.7)$ \\
Depression & Post Intervention & $13(43.3)$ & $7(23.3)$ & $10(33.3)$ \\
& Follow-up & $20(66.7)$ & $9(30.0)$ & $1(3.3)$ \\
\hline Mobility & Baseline & $13(43.3)$ & $9(30.0)$ & $8(26.7)$ \\
& Post Intervention & $15(50.0)$ & $14(46.7)$ & $1(3.3)$ \\
& Follow-up & $8(26.7)$ & $17(56.7)$ & $5(16.6)$ \\
\hline Pain / Discomfort & Baseline & $11(36.7)$ & $11(36.7)$ & $8(26.6)$ \\
& Post Intervention & $9(30.0)$ & $14(46.7)$ & $7(23.3)$ \\
& Follow-up & $12(40.0)$ & $13(43.3)$ & $5(16.7)$ \\
\hline Abbreviation: n: number of observations & & &
\end{tabular}

\section{Adverse Events}

During the IDE sessions performed by all patients, only $2.5 \%$ of the sessions ended up with complications and these included: 7 incidents of systolic hypertension, 1 incident of diastolic hypertension, 2 incidents of hypotension, and 2 incidents of leg cramps. In these cases, IDE was stopped immediately and when needed, medication was given by the medical team. Furthermore, exercise was mostly discontinued before the completion of the 45 minutes because most patients reported being tired, or had leg/knee pain.

\section{DISCUSSION}

To our knowledge, this is the first IDE program to be implemented in the UAE. The 6-month aerobic IDE program showed a tendency towards a positive effect of IDE on serum $P$ levels among the patients. However, no major effects on other parameters such as QOL and MIS could be identified. Around $25 \%$ of the patients in the HD unit were eligible and willing to participate in the study. Compared with other single group interventions of similar nature ((Musavian et al. 2015; Reboredo et al. 2010; Golebiowski et al. 2012; Parsons et al. 2006; Chigira et al. 2017; Bae et al. 2015; Bohm et al. 2014; Besnier et al. 2012; McMurray et al. 
2008), our sample number was the largest, with the exception of one retrospective study that gathered 102 patients (Parker et al. 2015).

In our intervention, the assessment of exercise intensity was subjective; originally, the protocol defined exercise intensity to be at the lowest level of moderate exercise intensity ( $40 \% \mathrm{VO}_{2} \mathrm{R}$ ) according to the American College of Sports Medicine guidelines (Garber et al. 2011). Unfortunately, most of the patients were on beta-blocker medication, which impedes the increase of the resting heart rate. Consequently, intensity was assessed using the perceived exertion rating (Borg Scale). Thus, our results could have reached significance if intensity was better controlled and if all patients exercised at a moderate intensity at all times. Nevertheless, our results on exercise intensity were close to those reported by a similar study conducted in Canada, where the Borg scale reported by the patients averaged 10.7 at baseline and 11.8 at 6 months (Bohm et al. 2014). The average monthly minutes of exercise per patient increased with time during our intervention, while the average patient exertion scale level remained almost constant. This reflects an improvement in the cardiovascular fitness of the participants. As for patient mean adherence level to the IDE, it reached 57.5\%, which was bit higher than the results from Bohm et al. (2014) (53\%). Out of the patients who exercised, 26.6 and $20 \%$ of the participants achieved moderate and high adherence to the program respectively, which is equivalent to the $26 \%$ moderately adherent but lower than the $41 \%$ highly adherent patients reported in Germany (Anding et al. 2015).

There was one month in the 6-month intervention that coincided with Ramadan - a religious event where Muslims fast during the day and eat after sunset. The experience of another study during this month (AlShamsi et al. 2016) reported that Ramadan fasters usually are young adults who missed more HD session and had higher serum $\mathrm{P}$ level then their older non fasting counterparts during Ramadan. However, in our sample, during this month, the mean patient minutes of exercise was at its highest, $96 \%$ of our Muslim patients chose to fast during Ramadan, adherence to the exercise session was higher than other months, and our patients were considered young (49 years). Thus, IDE may play a role in regulating serum $P$ for the fasting HD patients.

The current study showed that in hyperphosphatemic patients, IDE decreased the serum P level with a change approaching significance. In this study, following the KDIGO recommendations, the target serum P level was 3.5-5.5mg/dl, and hyperphosphatemia cut off point was $\geq 5.5 \mathrm{mg} / \mathrm{dl}$ (Ketteler et al. 2017). This cut off point was also used by Al-Qassimi hospital, where the study took place. Our results can be compared to those of Makhlough et al. (2012), where patient baseline serum $P$ was $7.68 \mathrm{mg} / \mathrm{dl}$ and decreased significantly after a 2 months of IDE to $5.83 \mathrm{mg} / \mathrm{dl}$. Furthermore, our findings were comparable to other single- 
group aerobic IDE interventions (Reboredo et al. 2010; McMurray et al. 2008), but not to Musavian et al. (2015), who were able to show a significant serum P decrease at the end of this intervention for the general HD population. We assume that hyperphosphatemics are the most that benefit from IDE programs and their number in our sample was small $(n=15)$ which did not allow the results to reach significance.

Similar to Musavian et al. (2015), our results showed no significant difference in dialysis efficacy assessed by Kt/v and URR. Other studies that achieved positive significant difference had, either a larger sample size (Parker et al. 2015), or a preconditioning phase (Reboredo et al. 2010) and/or a longer IDE session (Parsons et al. 2006). In our sample, MIS did not deteriorate, but given the absence of a control group we cannot assess that aerobic IDE helps in preventing the commonly reported deterioration of the nutritional status of HD patients over time (Karavetian et al. 2016).

In this intervention, the QOL was assessed using the EuroQO1-5D-5L; our results revealed the clinically positive effect of IDE on most domains of QOL post-intervention; more patients revealed no problem in the self-care, in performing daily activities, and in mobility; fewer patients reported suffering from anxiety, depression, pain and discomfort. This effect was less evident at follow-up. The reasoning for this may be that the IDE has to be maintained at all times to sustain the improvement of health. As for the VAS-QOL, it is stipulated that it was over-rated by the study participants who had strong religious beliefs, and their interpretation of sickness was seen as a cause of "fate" and "will of God"; which hindered their ability to rate the domain with objectivity and accuracy. It would have been a good idea to present the VAS without numbers to have more room for improvement. In fact, a more culturally specific QOL instrument should be customized for this population; this was also noted in another study conducted in a Middle Eastern Country (Karavetian et al. 2016). Other researches on aerobic IDE have used other QOL instruments in their evaluation. Accordingly, we could not compare the different QOL domains with other studies. However, single groups cycling IDE interventions conducted in various countries, and ranging from 30 to 70 minutes, have shown significant improvement in QOL total score or specific QOL domains (Musavian et al. 2015; Reboredo et al. 2010; Golebiowski et al. 2012).

Adverse events were encountered during the intervention in $2.5 \%$ of the sessions. Our results were comparable to Golebiowski et al. (2012), who had similar setting and recorded complications in $2 \%$ of the sessions. Moreover, none of the complications were acute or life threatening, they were the kind that could be managed within the HD unit. 
This study had some limitations; the design was non-randomized, and lacked a control group; thus clear conclusions could not be drawn. The subjective method used to measure intensity might have influenced the results.

\section{Implications for clinical practice}

This study had some implications for clinical practice. This intervention suggests that engaging in a 45 minutes of IDE exercise, 2-3 times per week for 6 months is safe and can be beneficial especially for hyperphosphatemic HD patients. Based on our experience, there are 3 major recommended components for IDE to be successfully implemented in an HD unit: 1) Equipment need to be kept in the treatment room for easy access; 2) Involvement of nursing staff of each HD unit to supervise the exercise program and assign specific coordinators for exercise to help the patient initiate the exercise safely and advise when to stop 3) And most important is the full endorsement of the unit's head nephrologist for the IDE program. Patient in the HD unit feel fragile and always need the reassurance of their nephrologist to start or proceed with any program.

In conclusion, the primary finding of the current study was that a moderate intensity aerobic intradialytic exercise program appeared to result in a clinically meaningful improvement in serum P level in hyperphosphatemic patients. No significant changes were observed in URR, MIS and in the VAS - QOL. Future investigations should focus on developing powered controlled trials to validate these observations. Today, we could say that IDE can promise clinical improvement in HD patients. 


\section{ACKNOWLEDGEMENTS}

We thank the nephrologist, Dr. Khaled Ibesh, for his daily supervision of the exercise program. We thank the physiotherapy department of Al Qassimi Hospital for their collaboration. We thank the nursing staff for their support and the research assistants for their assistance in data collection and exercise intervention. Special thank to the HD patients, who trusted us along the way.

Conflict of interest statement: The authors declare no conflict of interest.

Funding: This study was funded by Zayed University, Dubai, UAE. 


\section{REFERENCES}

1990. EuroQol--a new facility for the measurement of health-related quality of life. Health policy (Amsterdam, Netherlands) 16(3): 199-208.

Afshar, R., Shegarfy, L., Shavandi, N., and Sanavi, S. 2010. Effects of aerobic exercise and resistance training on lipid profiles and inflammation status in patients on maintenance hemodialysis. Indian journal of nephrology 20(4): 185-189. doi:10.4103/0971-4065.73442.

Alshamsi, S., Binsaleh, F., Hejaili, F., Karkar, A., Moussa, D., Raza, H., et al. 2016. Changes in biochemical, hemodynamic, and dialysis adherence parameters in hemodialysis patients during Ramadan. Hemodialysis international. International Symposium on Home Hemodialysis 20(2): 270-276. doi:10.1111/hdi.12369.

Amann, K., Gross, M.L., London, G.M., and Ritz, E. 1999. Hyperphosphataemia--a silent killer of patients with renal failure? Nephrology, dialysis, transplantation : official publication of the European Dialysis and Transplant Association - European Renal Association 14(9): 2085-2087.

Anding, K., Bar, T., Trojniak-Hennig, J., Kuchinke, S., Krause, R., Rost, J.M., et al. 2015. A structured exercise programme during haemodialysis for patients with chronic kidney disease: clinical benefit and long-term adherence. BMJ open 5(8): e008709. doi:10.1136/bmjopen-2015-008709.

Bae, Y.H., Lee, S.M., and Jo, J.I. 2015. Aerobic training during hemodialysis improves body composition, muscle function, physical performance, and quality of life in chronic kidney disease patients. Journal of physical therapy science 27(5): 1445-1449. doi:10.1589/jpts.27.1445.

Besnier, F., Laruelle, E., Genestier, S., Gie, S., Vigneau, C., and Carre, F. 2012. [Effects of exercise training on ergocycle during hemodialysis in patients with end stage renal disease: relevance of the anaerobic threshold intensity]. Nephrologie $\&$ therapeutique 8(4): 231-237. doi:10.1016/j.nephro.2011.10.002.

Bohm, C., Stewart, K., Onyskie-Marcus, J., Esliger, D., Kriellaars, D., and Rigatto, C. 2014. Effects of intradialytic cycling compared with pedometry on physical function in chronic outpatient hemodialysis: a prospective randomized trial. Nephrology, dialysis, transplantation : official publication of the European Dialysis and Transplant Association - European Renal Association 29(10): 1947-1955. 
doi:10.1093/ndt/gfu248.

Borg Rating of Perceived Exertion Scale. In: Centers for Disease Control and Prevention [online]. Available from www.cdc.gov/physicalactivity/basics/measuring/exertion.htm [accessed 17 September 2017].

Chigira, Y., Oda, T., Izumi, M., and Yoshimura, T. 2017. Effects of exercise therapy during dialysis for elderly patients undergoing maintenance dialysis. Journal of physical therapy science 29(1): 20-23. doi:10.1589/jpts.29.20.

Chiu, Y.W., Teitelbaum, I., Misra, M., de Leon, E.M., Adzize, T., and Mehrotra, R. 2009. Pill burden, adherence, hyperphosphatemia, and quality of life in maintenance dialysis patients. Clinical journal of the American Society of Nephrology : CJASN 4(6): 10891096. doi:10.2215/cjn.00290109.

Chung, Y.C., Yeh, M.L., and Liu, Y.M. 2017. Effects of intradialytic exercise on the physical function, depression and quality of life for haemodialysis patients: a systematic review and meta-analysis of randomised controlled trials. Journal of clinical nursing 26(13-14): 1801-1813. doi:10.1111/jocn.13514.

de Lima, M.C., Cicotoste Cde, L., Cardoso Kda, S., Forgiarini, L.A., Jr., Monteiro, M.B., and Dias, A.S. 2013. Effect of exercise performed during hemodialysis: strength versus aerobic. Renal failure 35(5): 697-704. doi:10.3109/0886022x.2013.780977.

Garber, C.E., Blissmer, B., Deschenes, M.R., Franklin, B.A., Lamonte, M.J., Lee, I.M., et al. 2011. American College of Sports Medicine position stand. Quantity and quality of exercise for developing and maintaining cardiorespiratory, musculoskeletal, and neuromotor fitness in apparently healthy adults: guidance for prescribing exercise. Medicine and science in sports and exercise 43(7): 1334-1359. doi:10.1249/MSS.0b013e318213fefb.

Giannaki, C.D., Sakkas, G.K., Karatzaferi, C., Hadjigeorgiou, G.M., Lavdas, E., Kyriakides, T., et al. 2013. Effect of exercise training and dopamine agonists in patients with uremic restless legs syndrome: a six-month randomized, partially double-blind, placebo-controlled comparative study. BMC nephrology 14: 194. doi:10.1186/14712369-14-194.

Golebiowski, T., Kusztal, M., Weyde, W., Dziubek, W., Wozniewski, M., Madziarska, K., et al. 2012. A program of physical rehabilitation during hemodialysis sessions improves 
the fitness of dialysis patients. Kidney \& blood pressure research 35(4): 290-296. doi:10.1159/000335411.

Haskell, W.L., Lee, I.M., Pate, R.R., Powell, K.E., Blair, S.N., Franklin, B.A., et al. 2007. Physical activity and public health: updated recommendation for adults from the American College of Sports Medicine and the American Heart Association. Medicine and science in sports and exercise 39(8): 1423-1434.

doi:10.1249/mss.0b013e3180616b27.

Ikizler, T.A. 2009. Dietary protein restriction in CKD: the debate continues. American journal of kidney diseases : the official journal of the National Kidney Foundation 53(2): 189-191. doi:10.1053/j.ajkd.2008.12.014.

Kalantar-Zadeh, K., Kopple, J.D., Humphreys, M.H., and Block, G. 2004. Comparing outcome predictability of markers of malnutrition-inflammation complex syndrome in haemodialysis patients. Nephrology, dialysis, transplantation : official publication of the European Dialysis and Transplant Association - European Renal Association 19(6): 1507-1519. doi:10.1093/ndt/gfh143.

Karavetian, M., Elzein, H., Rizk, R., Jibai, R., and de Vries, N. 2016. Nutritional education for management of osteodystrophy: Impact on serum phosphorus, quality of life, and malnutrition. Hemodialysis international. International Symposium on Home Hemodialysis 20(3): 432-440. doi:10.1111/hdi.12405.

Ketteler M, Block GA, Evenepoel P, et al. Executive summary of the 2017 KDIGO Chronic Kidney Disease-Mineral and Bone Disorder (CKD-MBD) Guideline Update: what's changed and why it matters. Kidney Int. 2017;92:26-36. Kidney international 92(6): 1558. doi:10.1016/j.kint.2017.10.001.

Kliger, A.S., Foley, R.N., Goldfarb, D.S., Goldstein, S.L., Johansen, K., Singh, A., et al. 2013. KDOQI US commentary on the 2012 KDIGO Clinical Practice Guideline for Anemia in CKD. American journal of kidney diseases : the official journal of the National Kidney Foundation 62(5): 849-859. doi:10.1053/j.ajkd.2013.06.008.

Maheshwari, V., Samavedham, L., and Rangaiah, G.P. 2011. A regional blood flow model for beta2-microglobulin kinetics and for simulating intra-dialytic exercise effect. Annals of biomedical engineering 39(12): 2879-2890. doi:10.1007/s10439-011-03835. 
Makhlough, A., Ilali, E., Mohseni, R., and Shahmohammadi, S. 2012. Effect of intradialytic aerobic exercise on serum electrolytes levels in hemodialysis patients. Iranian journal of kidney diseases 6(2): 119-123.

McMurray, A., Blazey, L., and Fetherston, C. 2008. The effect of intradialytic foot pedal exercise on blood pressure phosphate removal efficiency and health related quality of life in haemodialysis patients. Renal Society of Australasia Journal 4(2): 38-45.

Musavian, A.S., Soleimani, A., Masoudi Alavi, N., Baseri, A., and Savari, F. 2015. Comparing the effects of active and passive intradialytic pedaling exercises on dialysis efficacy, electrolytes, hemoglobin, hematocrit, blood pressure and healthrelated quality of life. Nursing and midwifery studies 4(1): e25922.

Orcy, R., Antunes, M.F., Schiller, T., Seus, T., and Bohlke, M. 2014. Aerobic exercise increases phosphate removal during hemodialysis: a controlled trial. Hemodialysis international. International Symposium on Home Hemodialysis 18(2): 450-458. doi:10.1111/hdi.12123.

Parker, K., Zhang, X., Lewin, A., and MacRae, J.M. 2015. The association between intradialytic exercise and hospital usage among hemodialysis patients. Applied physiology, nutrition, and metabolism $=$ Physiologie appliquee, nutrition et metabolisme 40(4): 371-378. doi:10.1139/apnm-2014-0326.

Parsons, T.L., Toffelmire, E.B., and King-VanVlack, C.E. 2006. Exercise training during hemodialysis improves dialysis efficacy and physical performance. Archives of physical medicine and rehabilitation 87(5): 680-687. doi:10.1016/j.apmr.2005.12.044.

Reboredo Mde, M., Henrique, D.M., Faria Rde, S., Chaoubah, A., Bastos, M.G., and de Paula, R.B. 2010. Exercise training during hemodialysis reduces blood pressure and increases physical functioning and quality of life. Artificial organs 34(7): 586-593. doi:10.1111/j.1525-1594.2009.00929.x.

Regolisti, G., Maggiore, U., Sabatino, A., Gandolfini, I., Pioli, S., Torino, C., et al. 2018. Interaction of healthcare staff's attitude with barriers to physical activity in hemodialysis patients: A quantitative assessment. PloS one 13(4): e0196313. doi:10.1371/journal.pone.0196313.

Rizk, R., Hiligsmann, M., Karavetian, M., and Evers, S.M. 2016. Economic evaluations of interventions to manage hyperphosphataemia in adult haemodialysis patients: A systematic review. Nephrology (Carlton, Vic.) 21(3): 178-187.

doi:10.1111/nep.12584. 
Salhab, N., Karavetian, M., Kooman, J., and Fiaccadori, E. 2018. Intradialytic Aerobic Exercise in the United Arab Emirates: a Descriptive Study. Arab Journal of Nutrition and Exercise 3(1): 18-34. doi:10.18502/ajne.v3i41.1693

Sheng, K., Zhang, P., Chen, L., Cheng, J., Wu, C., and Chen, J. 2014. Intradialytic exercise in hemodialysis patients: a systematic review and meta-analysis. American journal of nephrology 40(5): 478-490. doi:10.1159/000368722.

St. Peter, W.L., Wazny, L.D., Weinhandl, E., Cardone, K.E., and Hudson, J.Q. 2017. A Review of Phosphate Binders in Chronic Kidney Disease: Incremental Progress or Just Higher Costs? Drugs 77(11): 1155-1186. doi:10.1007/s40265-017-0758-5.

White, A., Odedina, F., Xiao, H., Campbell, E., and Segal, R. 2006. The Economic Burden of End-Stage Renal Disease with Hyperphosphatemia: A Study of Florida Medicaid. DISEASE MANAGEMENT AND HEALTH OUTCOMES 14(2): 99-106.

Wong, J., Davis, P., Patidar, A., Zhang, Y., Vilar, E., Finkelman, M., et al. 2017. The Effect of Intra-Dialytic Exercise on Inflammation and Blood Endotoxin Levels. Blood purification 44(1): 51-59. doi:10.1159/000455059.

Wu, Y., He, Q., Yin, X., He, Q., Cao, S., and Ying, G. 2014. Effect of individualized exercise during maintenance haemodialysis on exercise capacity and health-related quality of life in patients with uraemia. The Journal of international medical research 42(3): 718-727. doi:10.1177/0300060513509037.

Young, E.W., Akiba, T., Albert, J.M., McCarthy, J.T., Kerr, P.G., Mendelssohn, D.C., et al. 2004. Magnitude and impact of abnormal mineral metabolism in hemodialysis patients in the Dialysis Outcomes and Practice Patterns Study (DOPPS). American journal of kidney diseases : the official journal of the National Kidney Foundation 44(5 Suppl 2): 34-38. 

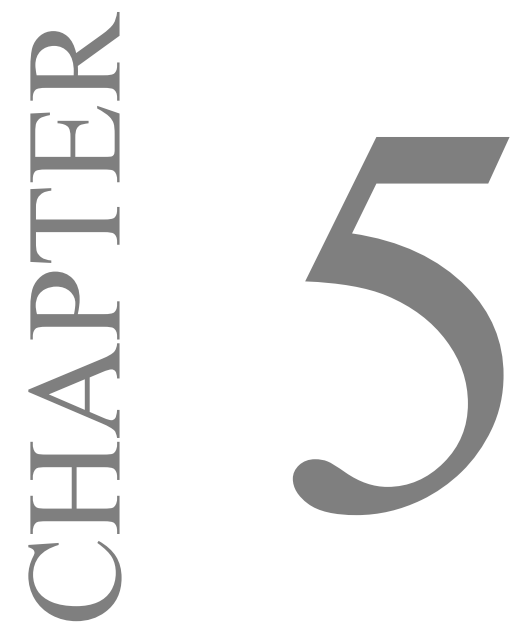

\section{ATTITUDES AND BARRIERS TO PHYSICAL ACTIVITY IN HEMODIALYSIS PATIENTS: \\ COULD INTRADIALYTIC EXERCISE MODIFY}

\section{THESE FACTORS?}

Salhab N, Kooman J, Fiaccadori E, Aljubori H, Karavetian M

Submitted 


\section{ABSTRACT}

Aim: This study aims to determine the impact of intradialytic exercise (IDE) on attitudes and barriers to physical activity (PA) in HD patients in the United Arab Emirates.

Methods: Forty-one adult HD patients from Al-Qassimi Hospital were recruited for a quasiexperimental intervention with pre-post evaluation. IDE patients trained for 45 minutes per HD session, 2-3 times per week, for 6 months on a static bicycle. Exercise intensity was assessed using the Borg Rating Scale. Participants were educated on the importance of exercise.

Results: 30 patients completed the study. The percentage of patients exercising was higher post intervention (86.7\%), but dropped to baseline at the follow up period. At post intervention and follow-up, patient's knowledge about the benefits and safety of exercise increased, with some patients facing no barrier to PA at the end of the study $(p=0.05)$. There was a significant increase in patients endorsing the "too many medical problems" barrier, and a significant decrease in patients endorsing the "can't afford to exercise" barrier.

Nephrologists and nurses acknowledge the importance of exercise in HD patients, but the former do not prescribe it.

Conclusion: Aerobic IDE and knowledge empowerment programs could help HD patients increase their knowledge about exercise benefits and safety and shed some barriers to exercise, although patients appear to easily fall back into their normal habits. Even after an IDE program was adopted in the unit, the medical team is still concerned about the risks of exercise in HD patients.

\section{Keywords:}

Attitude, exercise, hemodialysis, nephrologists, physical activity 


\section{INTRODUCTION}

Physical activity (PA) in hemodialysis (HD) patients is low compared to healthy sedentary controls reporting to exercise a maximum of once a week (Johansen et al., 2000). The poor physical performance is associated with the muscle atrophy accompanying the inflammatory processes of chronic kidney disease (CKD) (Johansen et al., 2003). To reverse muscle wasting, dietary intervention alone is not sufficient (Wang $\&$ Mitch, 2014). Thus emerges the need for intradialytic exercise (IDE) as a step to counteract sedentarism, which is associated with an increased risk of mortality among dialysis patients (O'Hare et al., 2003).

Unfortunately, HD patients face various barriers when it comes to the adoption of an IDE program. A recent review has identified fatigue and lack of energy as the most common barriers to exercise in CKD patients, followed by the burden of comorbidities and lack of time or access to exercise (Hanna \& Bronas, 2017). However, it is not solely the patients' call, as lack of motivation is also a major factor for patients not to exercise (Jhamb et al., 2016). Conversely, in HD patients not reporting barriers to exercise, the pro-active staff attitude proved to be effective in encouraging them to engage in PA programs (Regolisti et al., 2018). To date, in general, the medical team is not advocating exercise for HD patients, the lack of time being a major contributor to this fact (Regolisti et al., 2018; Fiaccadori et al., 2014), in addition to the lack of confidence in the absence of guidelines (Regolisti et al., 2018).

In an initiative to overcome patients' barriers to exercise, Tentori et al. (2010) highlighted that HD patients have higher chances of exercising in units offering exercise programs. Particularly, HD patients, who had previously engaged in an exercise program, reported to be more positive towards exercise than their counterparts with no exercise exposure (Jayaseelan, 2018). Furthermore, a study showed that home-based exercise program decreased the number of existing barriers towards exercise (Tao et al., 2017).

The current study assessed the effects of a 6-months supervised IDE program on HD patients' barriers to PA, intradialytic or interdialytic exercise behavior, attitude, and knowledge in the United Arab Emirates (UAE). It also aimed to highlight the attitudes of the nephrologist and dialysis nurses towards integrating PA in HD patient's lifestyles. 


\section{MATERIAL AND METHODS}

\section{Study Design and Participants}

A 6-month, quasi-experimental intervention was conducted on all eligible and consenting patients from the HD unit in Al Qassimi Hospital, UAE. All procedures performed were in accordance with the ethical standards of the 1964 Helsinki declaration and its later amendments or comparable ethical standards. The study was approved by the Research Ethics Committees of the Ministry of Health and Prevention of the UAE (MOHP/DXB/SUBC/NO-5/2016) and by Zayed University (ZU15_118_F). Informed consent of all eligible patients was obtained before the initiation of the study. The study protocol and baseline results have been previously published (chapter 3) (Salhab et al., 2018).

\section{Study protocol}

The study lasted 12 months, and was divided into 3 stages:

1. Baseline $\left(\mathrm{T}_{0}\right)$ : The research team verified the planned intervention methods on 10 randomly chosen patients from the HD unit and offered a 1-month individualized patient education on the importance of exercise to their health. Patients were also introduced to the concept of IDE. Baseline assessment was conducted in the last 2 weeks of $\mathrm{T}_{0}$.

2. Intervention $\left(\mathrm{T}_{1}\right)$ : All consenting participants conducted the IDE in a supine position on a static pedaler "Pedal Exerciser KD" within the first 2 hours of dialysis for 45 minutes, 2-3 times per week, for 6 months. IDE sessions started with a 2-minute warm up, cycling at the lowest resistance of the pedaler (Wong et al., 2017). Then, patients were advised to continue cycling at a moderate intensity level of exercise matching the level 12 on the Borg Rating of Perceived Exertion Scale (Wong et al., 2017; Center for Disease Control and Prevention). Exercise intensity, duration, patient's complaints and blood pressure (BP) were recorded by the research team throughout each IDE session; in case of BP $>200 / 110 \mathrm{mmHg}$, exercise was not initiated or was stopped (de Lima et al., 2013). Patients were under the direct supervision of the medical team; in case of any discomfort, the intervention was immediately halted. In addition, patients received monthly one-to-one education on the benefits of exercise for HD patients focusing on its effect on dialysis efficiency and hyperphosphatemia management; moreover, ideas on safe workout modes and integration of exercise in their regular routine were provided. The education material was given in oral and written forms to patients, and illustrated on a poster in the HD unit. 
3. Follow-up $\left(\mathrm{T}_{2}\right)$ : After the IDE intervention, the research team refrained from seeing the patients for 3 months. Thereafter, all patients were assessed on the study outcome measures; the latter took 1 month.

\section{Outcome Measures}

Three questionnaires were used in this study:

1. Exercise behavior, attitude and knowledge questionnaire developed by the authors of the study; based on the theory of planned behavior model (Ajzen \& Fishbein, 1988). It included 7 questions with multiple-choice answers (Appendix 1). The behavior questions assessed patients' level of PA, whether the activity is performed during dialysis (intradialytic) or outside the dialysis unit (interdialytic). Thus, it included the IDE related to our intervention.

2. Patients' barriers to PA were assessed using a questionnaire adapted from Fiaccadori et al. (2014). It included 24 questions assessing psychological, physical, and economical barriers, in addition to the lack of time factor and comorbidities. We removed the question on amputation, as this was an exclusion criterion in our study. Patients were considered as having a barrier if they experienced it "sometimes", "often" or "always"; if their reply was "never, patients were considered as not having this barrier. The final version of the questionnaire was shared and approved by Fiaccadori et al. (2014).

3. Attitudes and practices of nephrologist and nurses towards PA conducted by HD patients were evaluated using a questionnaire developed by Fiaccadori et al. (2014). It included 17 questions for the nephrologists and 13 questions for the nurses. Responders had to choose yes or no answers for each question. This questionnaire was administered at $\mathrm{T}_{1}$.

\section{Sample Size}

GPower 3.1 software was used for sample size estimation; based on the change of serum phosphorus; since the latter was the primary outcome variable of the mother study. The needed sample size was $n=34$, assuming a two-sided type I error rate of $5 \%$, a power of $80 \%$ and an effect size of $\mathrm{d}=|0.56|$; this calculation was based on the findings of Makhlough et al. (2012). Out of 57 eligible patients, 41 consented and were included in the analysis.

\section{Data Collection}

The research team conducted the questionnaires and collected demographics and blood tests results from the patients' medical files. All study outcome measures were collected at 3 time-points: $\mathrm{T}_{0}-\mathrm{T}_{1}-\mathrm{T}_{2}$. 


\section{Statistical Analysis}

For statistical analysis, we used the Statistical Package for the Social Sciences (SPSS), version 21 (IBM Corp., Armonk, NY, USA). Categorical and continuous data were summarized using descriptive statistics, and results were presented as frequencies and percentages for categorical variables, and means and standard deviations for continuous ones. Cochran's Q Test was used to analyze the differences in barriers to PA between the 3 study timelines. Significance was accepted at $p \leq 0.05$.

\section{RESULTS}

\section{Characteristics of the patients}

Forty-one patients were eligible and consented to enter the study, out of whom 30 patients completed the study. Reasons for patient's drop out were as follows: unsatisfaction with the static pedaler, transfer to another dialysis unit, transplantation, and death. Half of the patients were males, relatively young ( $48.87 \pm 11.29$ years), and $73.4 \%$ of them were of Arab origin. Hypertension was prevalent in $93.3 \%$ of the sample, followed by diabetes $(53.3 \%)$, and cardiovascular disease (13.3\%). Full description of total sample characteristics at baseline is detailed in an earlier publication (Salhab et al., 2012).

\section{Behavior, attitude and knowledge to exercise}

The number of patients exercising either during dialysis or not increased by $20 \%$ at $\mathrm{T}_{1}$ and this change dropped back completely at $\mathrm{T}_{2}(\mathrm{p}=0.07)$. The percentage of patient exercising $>30$ minutes remained almost the same at the 3 time-points of the study, with walking being the primary exercise at $\mathrm{T}_{0}(100 \%)$ and $\mathrm{T}_{2}(85 \%)$, yet cycling being dominant at $\mathrm{T}_{1}(84.6 \%)$, which was the exercise adapted for the IDE intervention. All patients reporting to cycle at $\mathrm{T}_{1}$ referred to the IDE intervention program. At $\mathrm{T}_{2}$, only $25 \%$ of the patients continued the cycling exercise at home on a personal static pedaler.

Throughout the study, the number of patients not intending to exercise remained constant (4 out of 30 patients), and the majority of the patients agreed on the importance of exercise for their health $(\mathrm{p}=0.22)$.

The percentage of patients having the correct knowledge about the safety of exercise increased by almost $30 \%$ at $\mathrm{T}_{1}$ and remained so at $\mathrm{T}_{2}(\mathrm{p}=0.26)$. Similarly, the patients having the correct knowledge about the benefits of exercise increased significantly by almost $40 \%$ at 
$\mathrm{T}_{1}(96.7 \%)$ and this was maintained at $\mathrm{T}_{2}(\mathrm{p}=0.0)$. Data on exercise behavior, attitude and knowledge of our sample are detailed in table 1.

Table 1 Exercise Behavior, Attitude \& Knowledge Questionnaire

\begin{tabular}{|c|c|c|c|c|}
\hline & $\begin{array}{c}\mathrm{T}_{0} \\
\mathrm{n}(\%)\end{array}$ & $\begin{array}{c}\mathrm{T}_{1} \\
\mathrm{n}(\%) \\
\end{array}$ & $\begin{array}{c}\mathrm{T}_{2} \\
\mathrm{n}(\%) \\
\end{array}$ & $\begin{array}{c}\mathrm{p} \\
\text { value }\end{array}$ \\
\hline \multicolumn{5}{|l|}{ Frequency of exercise per week } \\
\hline Don't exercise & $10(33.3)$ & $4(13.3)$ & $10(33.3)$ & \multirow[b]{2}{*}{0.07} \\
\hline Exercise 1 or more times / week & $20(66.7)$ & $26(86.7)$ & $20(66.7)$ & \\
\hline \multicolumn{5}{|c|}{ Attitude towards exercise importance on Health ${ }^{\dagger(\text { among full sample) }}$} \\
\hline Important & $29(96.7)$ & $30(100)$ & $28(93.3)$ & \multirow[b]{2}{*}{0.22} \\
\hline Not Important & $1(3.3)$ & $0(0.0)$ & $2(6.7)$ & \\
\hline \multicolumn{5}{|c|}{ Knowledge about exercise safety for HD patients ${ }^{\dagger}$} \\
\hline Correct knowledge & $16(53.3)$ & $24(80)$ & $25(83.3)$ & \multirow[b]{2}{*}{0.26} \\
\hline False knowledge & $14(46.6)$ & $6(20)$ & $5(16.7)$ & \\
\hline \multicolumn{5}{|c|}{ Knowledge about the benefits of exercise for HD patients ${ }^{\dagger *}$} \\
\hline Correct knowledge & $17(56.7)$ & $29(96.7)$ & $27(90)$ & \multirow[b]{2}{*}{$0.00^{*}$} \\
\hline No knowledge & $13(43.3)$ & $1(3.3)$ & $3(10)$ & \\
\hline
\end{tabular}

\section{Barriers to physical activity}

There was a significant increase in patients reporting to have "too many medical problems" throughout the study phases. Also, there was a significant decrease in patients reporting that they "can't afford to exercise". There was no significant difference on the rest of the barriers assessed. Table 2 illustrates the perceived barriers to PA.

At $\mathrm{T}_{0}$, all patients had at least 1 barrier to perform exercise. There was a $15 \%$ significant increase $(\mathrm{p}=0.05)$ in the number of patients who had 0 barrier to exercise at $\mathrm{T}_{1} \&$ $\mathrm{T}_{2}$. Patients with 2 - 4 barriers to exercise decreased by $3 \%$ at $\mathrm{T}_{1}$ and by almost $15 \%$ at $\mathrm{T}_{2}$ $(\mathrm{p}=0.26)$. And those with 5 - 9 barriers to exercise remained constant $(36.7 \%)$ throughout the study $(\mathrm{p}=1.00)$. Finally, at $\mathrm{T}_{2}$, patients reporting $\geq 10$ barriers doubled $(26.7 \%)$ compared to $\mathrm{T}_{0}$ and $\mathrm{T}_{1}(\mathrm{p}=0.20)$.

The most frequently reported barrier at $\mathrm{T}_{0}$ was "fatigue on dialysis days" $(63.3 \%)$ followed by "fear of getting hurt" (36.7\%). At $\mathrm{T}_{1}$, the most frequently reported barrier was "too many medical problems" (46.7\%) followed by "fatigue on dialysis days" (43.3\%). At the end of the study, at $\mathrm{T}_{2}$, patients were left with 4 main barriers: "fatigue on dialysis days" (63.3\%), "too 
many medical problems (63.6\%), "pain on dialysis days" (53.3\%), and "fatigue on non-dialysis days" $(50 \%)$.

Table 2. Perceived barriers to physical activity

\begin{tabular}{|c|c|c|c|c|}
\hline Barriers & $\begin{array}{c}\mathrm{T}_{0} \\
\mathrm{n}(\%)\end{array}$ & $\begin{array}{c}\mathrm{T}_{1} \\
\mathrm{n}(\%) \\
\end{array}$ & $\begin{aligned} \mathrm{T}_{2} \\
\mathrm{n}(\%) \\
\end{aligned}$ & $\mathrm{p}$ value \\
\hline Fatigue on dialysis days & $19(63.3)$ & $13(43.3)$ & $19(63.3)$ & 0.07 \\
\hline Fatigue on non-dialysis days & $10(33.3)$ & $10(33.3)$ & $15(50)$ & 0.1 \\
\hline Pain on dialysis days & $8(26.7)$ & $12(40)$ & $16(53.3)$ & 0.06 \\
\hline Pain on non-dialysis days & $6(20)$ & $9(30)$ & $14(46.7)$ & 0.06 \\
\hline Too many medical problems & $9(30)$ & $14(46.7)$ & $19(63.3)$ & $0.02 *$ \\
\hline Fear of getting hurt & $11(36.7)$ & $9(30)$ & $11(36.7)$ & 0.77 \\
\hline Lack of time on dialysis days & $7(23.3)$ & $5(16.7)$ & $7(23.3)$ & 0.69 \\
\hline Lack of time on non-dialysis days & $4(13.3)$ & $5(16.7)$ & $3(10)$ & 0.74 \\
\hline $\begin{array}{l}\text { Lack of time because of too many medical } \\
\text { appointments }\end{array}$ & $4(13.3)$ & $4(13.3)$ & $4(13.3)$ & 1.00 \\
\hline Physician concern & $4(13.3)$ & $1(3.3)$ & $2(6.7)$ & 0.24 \\
\hline Shortness of breath & $9(30)$ & $9(30)$ & $9(30)$ & 1.00 \\
\hline Chest pain & $8(26.7)$ & $6(20)$ & $6(20)$ & 0.69 \\
\hline Feelings of helplessness & $7(23.3)$ & $6(20)$ & $6(20)$ & 0.92 \\
\hline Not wanting to be seen doing exercise & $1(3.3)$ & $3(10)$ & $1(3.3)$ & 0.36 \\
\hline Ulcers on legs and feet & $9(30)$ & $7(23.3)$ & $4(13.3)$ & 0.20 \\
\hline Feeling too old & $2(6.7)$ & $6(20)$ & $5(16.7)$ & 0.19 \\
\hline No exercise partner & $3(10)$ & $7(23.3)$ & $5(16.7)$ & 0.39 \\
\hline 'I don't want to' & $4(13.3)$ & $7(23.3)$ & $3(10)$ & 0.33 \\
\hline Sadness & $7(23.3)$ & $8(26.7)$ & $7(23.3)$ & 0.93 \\
\hline Family concern & $4(13.3)$ & 0 & $3(10)$ & 0.11 \\
\hline Can't afford to exercise & $8(26.7)$ & $2(6.7)$ & $2(6.7)$ & $0.02 *$ \\
\hline Inability to travel & $7(23.3)$ & $3(10)$ & $3(10)$ & 0.16 \\
\hline No place to exercise & $3(10)$ & $3(10)$ & $3(10)$ & 1.00 \\
\hline Lack of safe place for exercise & $4(13.3)$ & $8(26.7)$ & $6(20)$ & 0.42 \\
\hline Percentage of patients endorsing no barriers & $0(0.0)$ & $4(13.3)$ & $5(16.7)$ & $0.05 *$ \\
\hline Percentage of patients endorsing 1 barrier & $5(16.7)$ & $2(6.6)$ & $1(3.3)$ & 0.15 \\
\hline Percentage of patients endorsing 2-4 barriers & $10(33.3)$ & $9(30)$ & $5(16.7)$ & 0.26 \\
\hline Percentage of patients endorsing 5-9 barriers & $11(36.7)$ & $11(36.7)$ & $11(36.7)$ & 1.00 \\
\hline $\begin{array}{l}\text { Percentage of patients endorsing } \geq 10 \\
\text { barriers }\end{array}$ & $4(13.3)$ & $4(13.3)$ & $8(26.7)$ & 0.20 \\
\hline
\end{tabular}


Attitudes of nephrologist and nurses towards exercise in hemodialysis patients

Four nephrologists filled the questionnaire. All of them acknowledged the importance of exercise for HD patients, were confident and had the time to discuss this issue during consultation. However, 3 out of 4 nephrologists were concerned about the risks of exercise for this population and thus do not prescribe it in their practice (table 3).

Nineteen nurses filled the questionnaire. All of them acknowledged the importance of exercise for HD patients. Most of the nurses (89\%) were confident to discuss the topic with the patients, and reported often asking and counseling patients about PA. However, $84 \%$ felt that counseling is part of the physician's role (table 3 ).

Table 3. Attitudes of the medical team towards physical activity in HD patients

\begin{tabular}{lcc}
\hline & Nephrologist & $\begin{array}{c}\text { HD } \\
\text { Nurses }\end{array}$ \\
\hline 1. Physical inactivity is an important health risk in the general & $4 / 4$ & $19 / 19$ \\
population & $4 / 4$ & $19 / 19$ \\
2. Increasing physical activity is beneficial for most people & $4 / 4$ & $19 / 19$ \\
3. Physical activity is beneficial for HD patients & $3 / 4$ & $18 / 19$ \\
4. I am concerned about the risks of exercise in HD patients & $3 / 4$ & $18 / 19$ \\
5. I believe that most HD patients would increase physical & & \\
activity if advised to do so & $4 / 4$ & $18 / 19$ \\
6. I have time to talk to HD patients about physical activity & $3 / 4$ & $18 / 19$ \\
7. I think HD patients are interested in the topic of physical & & \\
activity & $4 / 4$ & $19 / 19$ \\
8. I think exercise is important (or is as important as other & & \\
medical issues) & $4 / 4$ & $16 / 19$ \\
9. I think it is the role of the physician to counsel HD patients & & $17 / 19$ \\
about physical activity & $4 / 4$ & $17 / 19$ \\
10. I feel confident in my ability to discuss this topic with patients & $2 / 4$ & $18 / 19$ \\
11. I often ask HD patients about physical activity & $3 / 4$ \\
12. I often counsel HD patients about physical activity & $3 / 4$ & $17 / 19$ \\
13. I often ask and counsel HD patients about physical activity & & \\
& & \\
Addressed only to Nephrologist & $1 / 4$ & - \\
14. I often prescribe HD patients about physical activity & $0 / 4$ & - \\
15. I often provide written material to HD patients about physical & & - \\
activity & $2 / 4$ & - \\
16. I often refer HD patients to physiotherapist & $0 / 4$ & \\
17. I often provide equipment HD patients for exercise & &
\end{tabular}




\section{DISCUSSION}

In the present study, we highlighted the change in patients' perceived barriers to PA following a 6-months IDE program in the UAE. The main finding was that "fatigue on dialysis days" remained the main barrier to PA; as for the chief success of the study, it was the ability of 6-month IDE to remove all barriers to PA from almost $15 \%$ of the sample population $(\mathrm{p}=0.05)$. The study also unveiled the positive attitudes of the medical team towards exercise in HD patients in the UAE.

In this study, 33.3\% reported not to exercise before the intervention, which was quite similar to the $38.1 \%$ reported from 2 HD centers in Italy (Bossola et al., 2014), but lower than the $43.9 \%$ reported internationally from the Dialysis Outcomes and Practice Patterns Study DOPPS $^{9}$, and the $>50 \%$ inactive patients in the study of Fiaccadori et al. (2014). Elderly HD patients are more prone to physical frailty and thus decreased physical functioning (Johansen et al., 2007). Therefore, the higher percentage of exercising patients in our sample may be due to their younger age.

Based on our results, a 6-months IDE program along with a monthly one to one patient education on exercise was sufficient to acquire and maintain the given knowledge at the end of the study $(\mathrm{p}=0.00)$. However, the intervention was not enough to sustain the exercise behavioral change. Our protocol did not assess patients' readiness to engage in a structured exercise program at baseline; thus each patient could have been in a different stage of readiness as suggested by the Transtheoretical Model (TTM) (Prochaska \& Velicer, 1997). After 1 month of education, our patients were directly put into the "action" phase; thus we assume that the duration given was not enough for all patients to sustain their behavior.

"Fatigue on dialysis days" continued to be the most identified barrier at the end of this study; a finding common in 5 other studies reporting on the prevalence of barriers to exercise in Italy, Canada and the USA (Jhamb et al., 2016; Fiaccadori et al., 2014; Bossola et al., 2014; Delgado \& Johansen, 2012; Kontos et al., 2007). Moreover, in Jordan, a country more comparable to UAE, "tiredness" and "lower extremity fatigue" was noted as the focal barriers to exercise (Darawad \& Khalil, 2013).

In the existing literature, few patients (3 - 6\%) from different backgrounds endorsed only 1 barrier at the time of assessment (Fiaccadori et al., 2014; Delgado \& Johansen, 2012); this was the case in the UAE after the completion of the IDE program. At the end of our study, the main barriers remaining with the patients were related to their medical problems and the fatigue/pain due to the dialysis session, which are inevitable consequences of the normal progression of end stage renal disease (Bossola \& Tazza, 2016; Davison, 2003); the 
frequency of reporting some of these barriers increased during the intervention. In fact, with the acquired knowledge about the benefits and safety of exercise, patients were unconsciously looking for an excuse for their exercise behavior at $\mathrm{T}_{2}$; "Too many medical problems" seemed to be the most logical fact and thus barrier that we could not change. Also, we observed a significant decrease in the "can't afford to exercise" barrier; in fact, the provided education introduced safe and inexpensive exercising modes for HD patients.

In the current study, 4 out of 5 nephrologists filled the questionnaire. Similarly to the results from Delgado and Johansen (2010) study, all of the nephrologists agreed that exercise is beneficial but most would not prescribe it. Also, all nephrologists agreed that exercise is as important as other medical issues versus $61.2 \%$ in Regolisti et al. (2018) study and $92.7 \%$ in Delgado and Johansen (2010) study. Overall, 75\% of the nephrologists were concerned about the risks of exercise compared to $40 \%$ reported by Delgado and Johansen (2010). The number of participants in Delgado and Johansen's study was much larger $(\mathrm{n}=198)$ (Delgado \& Johansen, 2010); thus, our small sample size could have biased the results. Note that results from a small sample size study were the opposite of ours where $5 / 5$ nephrologists did not consider exercise as important as other issue and were not confident to discuss the topic with patients, but $4 / 5$ nephrologists prescribed it (Fiaccadori et al., 2014). On the other hand, results from a study in the UK comparing barriers before and after experiencing an IDE program, revealed "staff workload" and "lack of time" to be the main barriers to exercise reported by both staff and patients at the 2 points in time (Young et al., 2015). In our program, the research team conducted the study and the hospital staff was not directly involved, thus we postulate that their answers were more theoretical and ideal.

One could question whether a lack of guidelines refrains the medical community from counseling the patients. In a study conducted by Delgado and Johansen (2010), nephrologists counseling behavior did not increase despite the Kidney Disease Outcomes Quality Initiative (KDOQI) cardiovascular guidelines recommendations for nephrologist to counsel their patients to increase PA (Delgado \& Johansen, 2010). In our study, 75\% of the nurses and $95 \%$ of the nephrologists reported to be counseling their patients for exercise. Also, the lack of experience with equipment could provide a barrier to implementation of an IDE program. In Kontos et al. (2007), nursing staff had trouble placing the heavy machinery to initiate the IDE, thus, they did not encourage their patients to exercise. According to DOPPS, patients' exercising status is influenced by both patient's characteristics and the provision of appropriate exercise equipment at the HD unit (Tentori et al., 2010). Additionally Johansen et al. (2003) reported low counseling levels in nephrologists facing lack of time, lack of confidence to counsel, lack of conviction that patients will respond and who believed that 
other medical problems were more important (Johansen et al., 2003). Staff reports from different facilities considered the lack of time as a constricting factor to promote PA (Regolisti et al., 2018; Fiaccadori et al., 2014; Kontos et al., 2007). Also, dialysis staff believed that only a program requiring minimal staff assistance could be feasible ${ }^{6}$, although in our study, $95 \%$ of the nurses stated that they have time to talk about PA. Similar results were noted from the USA, where $76 \%$ of the nurses have the time to discuss this topic in their daily routine (Painter et al., 2004). In Al Qassimi Hospital, the nurse to patient ratio is 1:4 and nurses were compassionate and liked to entertain the patients. However, one may postulate that, in certain HD units, the lack of time could be the reason for not adopting IDE programs.

A limitation of our study is the absence of a comparative control group. We tried to attenuate this limitation by comparing the change in the same group at 3 points in time. Another limitation is the subjective tools used to assess the outcomes of the study. Perhaps using more tangible assessment methods for patient's exercise capacity and endurance or measuring patient's body composition would have allowed us to draw firmer conclusions. Finally, the young age of the enrolled patients is a limitation; the inclusion criteria for HD patients to enter an IDE program might have led to the exclusion of the elderly group in this sample population.

\section{CONCLUSION}

Aerobic IDE and one to one counseling may be associated with an increase in knowledge towards exercise in HD patients. Additionally, it may help some patients overcome their barriers to exercise. Although the nephrologists acknowledge the importance of exercise for HD patients, they are still refrained from prescribing it, as they are concerned about its risks. If adopted in HD treatment protocol, IDE could be part of the multidisciplinary approach to decrease the treatment burden of HD patients.

\section{ACKNOWLEDGEMENTS}

This study was funded by Zayed University, Dubai, UAE, and registered in the ClinicalTrials.gov (ID: NCT03131804). The authors would like to thank the dialysis unit nephrologists and nurses for their support and participation, as well as the physiotherapists at Al Qassimi Hospital for their collaboration. We thank the research assistants for their help in data collection and exercise intervention. Our deepest appreciation goes to the participating patients. 


\section{REFERENCES}

Ajzen, I., \& Fishbein, M. (1988). Theory of reasoned action - theory of planned behavior. University of South Florida

Bossola, M., Pellu, V., Di Stasio, E., Tazza, L., Giungi, S., \& Nebiolo, P. E. (2014). Selfreported physical activity in patients on chronic hemodialysis: correlates and barriers. Blood Purif, 38(1), 24-29. doi:10.1159/000363599

Bossola, M., \& Tazza, L. (2016). Postdialysis Fatigue: A Frequent and Debilitating Symptom. Semin Dial, 29(3), 222-227. doi:10.1111/sdi.12468

Centers for Disease Control and Prevention. Borg Rating of Perceived Exertion Scale. Available from:https://www.cdc.gov/physicalactivity/basics/measuring/exertion.htm.

Darawad, M. W., \& Khalil, A. A. (2013). Jordanian dialysis patients' perceived exercise benefits and barriers: a correlation study. Rehabil Nurs, 38(6), 315-322. doi: $10.1002 / \mathrm{rnj} .98$

Davison, S. N. (2003). Pain in hemodialysis patients: prevalence, cause, severity, and management. Am J Kidney Dis, 42(6), 1239-1247.

de Lima, M. C., Cicotoste Cde, L., Cardoso Kda, S., Forgiarini, L. A., Jr., Monteiro, M. B., \& Dias, A. S. (2013). Effect of exercise performed during hemodialysis: strength versus aerobic. Ren Fail, 35(5), 697-704. doi:10.3109/0886022x.2013.780977

Delgado, C., \& Johansen, K. L. (2010). Deficient counseling on physical activity among nephrologists. Nephron Clin Pract, 116(4), c330-336. doi:10.1159/000319593

Delgado, C., \& Johansen, K. L. (2012). Barriers to exercise participation among dialysis patients. Nephrol Dial Transplant, 27(3), 1152-1157. doi:10.1093/ndt/gfr404

Fiaccadori, E., Sabatino, A., Schito, F., Angella, F., Malagoli, M., Tucci, M., . . Regolisti, G. (2014). Barriers to physical activity in chronic hemodialysis patients: a singlecenter pilot study in an Italian dialysis facility. Kidney Blood Press Res, 39(2-3), 169175. doi: $10.1159 / 000355793$

Hannan, M., \& Bronas, U. G. (2017). Barriers to exercise for patients with renal disease: an integrative review. $J$ Nephrol, 30(6), 729-741. doi:10.1007/s40620-017-0420-z

Jayaseelan G. (2018). Exercise benefits and barriers: the perceptions of people receiving hemodialysis. Nephrol Nurs J. 45(2), 185-219 
Jhamb, M., McNulty, M. L., Ingalsbe, G., Childers, J. W., Schell, J., Conroy, M. B., . . Dew, M. A. (2016). Knowledge, barriers and facilitators of exercise in dialysis patients: a qualitative study of patients, staff and nephrologists. BMC Nephrol, 17(1), 192. doi:10.1186/s12882-016-0399-z

Johansen, K. L., Chertow, G. M., Jin, C., \& Kutner, N. G. (2007). Significance of frailty among dialysis patients. J Am Soc Nephrol, 18(11), 2960-2967. doi:10.1681/asn.2007020221

Johansen, K. L., Chertow, G. M., Ng, A. V., Mulligan, K., Carey, S., Schoenfeld, P. Y., \& Kent-Braun, J. A. (2000). Physical activity levels in patients on hemodialysis and healthy sedentary controls. Kidney Int, 57(6), 2564-2570. doi:10.1046/j.15231755.2000.00116.x

Johansen, K. L., Sakkas, G. K., Doyle, J., Shubert, T., \& Dudley, R. A. (2003). Exercise counseling practices among nephrologists caring for patients on dialysis. Am J Kidney Dis, 4l(1), 171-178. doi:10.1053/ajkd.2003.50001

Johansen, K. L., Shubert, T., Doyle, J., Soher, B., Sakkas, G. K., \& Kent-Braun, J. A. (2003). Muscle atrophy in patients receiving hemodialysis: effects on muscle strength, muscle quality, and physical function. Kidney Int, 63(1), 291-297. doi:10.1046/j.15231755.2003.00704.x

Kontos, P. C., Miller, K. L., Brooks, D., Jassal, S. V., Spanjevic, L., Devins, G. M., . . . Naglie, G. (2007). Factors influencing exercise participation by older adults requiring chronic hemodialysis: a qualitative study. Int Urol Nephrol, 39(4), 1303-1311. doi:10.1007/s11255-007-9265-Z

Makhlough, A., Ilali, E., Mohseni, R., \& Shahmohammadi, S. (2012). Effect of intradialytic aerobic exercise on serum electrolytes levels in hemodialysis patients. Iran J Kidney Dis, 6(2), 119-123.

O'Hare, A. M., Tawney, K., Bacchetti, P., \& Johansen, K. L. (2003). Decreased survival among sedentary patients undergoing dialysis: results from the dialysis morbidity and mortality study wave 2. Am J Kidney Dis, 41(2), 447-454. doi:10.1053/ajkd.2003.50055

Painter, P., Carlson, L., Carey, S., Myll, J., \& Paul, S. (2004). Determinants of exercise encouragement practices in hemodialysis staff. Nephrol Nurs $J, 31(1), 67-74$.

Prochaska, J. O., \& Velicer, W. F. (1997). The transtheoretical model of health behavior change. Am J Health Promot, 12(1), 38-48. doi:10.4278/0890-1171-12.1.38 
Regolisti, G., Maggiore, U., Sabatino, A., Gandolfini, I., Pioli, S., Torino, C., . . Fiaccadori, E. (2018). Interaction of healthcare staff's attitude with barriers to physical activity in hemodialysis patients: A quantitative assessment. PLoS One, 13(4), e0196313. doi:10.1371/journal.pone.0196313

Salhab, N., Karavetian, M., Kooman, J., Fiaccadori, E. (2018). Intradialytic Aerobic Exercise in the United Arab Emirates: a Descriptive Study. AJNE, 3(1), 18-34.

Tao, X., Chow, S. K. Y., \& Wong, F. K. (2017). The effects of a nurse-supervised home exercise programme on improving patients' perceptions of the benefits and barriers to exercise: A randomised controlled trial. J Clin Nurs, 26(17-18), 2765-2775. doi:10.1111/jocn.13798

Tentori, F., Elder, S. J., Thumma, J., Pisoni, R. L., Bommer, J., Fissell, R. B., . . Robinson, B. M. (2010). Physical exercise among participants in the Dialysis Outcomes and Practice Patterns Study (DOPPS): correlates and associated outcomes. Nephrol Dial Transplant, 25(9), 3050-3062. doi:10.1093/ndt/gfq138

Wang, X. H., \& Mitch, W. E. (2014). Mechanisms of muscle wasting in chronic kidney disease. Nat Rev Nephrol, 10(9), 504-516. doi:10.1038/nrneph.2014.112

Wong, J., Davis, P., Patidar, A., Zhang, Y., Vilar, E., Finkelman, M., \& Farrington, K. (2017). The Effect of Intra-Dialytic Exercise on Inflammation and Blood Endotoxin Levels. Blood Purif, 44(1), 51-59. doi:10.1159/000455059

Young, H. M., Hudson, N., Clarke, A. L., Dungey, M., Feehally, J., Burton, J. O., \& Smith, A. C. (2015). Patient and Staff Perceptions of Intradialytic Exercise before and after Implementation: A Qualitative Study. PLoS One, 10(6), e0128995. doi:10.1371/journal.pone.0128995 


\section{Appendix 1}

EXERCISE BEHAVIOR QUESTIONNAIRE CIRCLE THE CORRECT ANSWER

SEX

1. Male

2. Female

HOW MANY DAYS PER WEEK DO YOU EXERCISE?

1. I don't exercise

2. I time per week

3. 2 times per week

4. 3 times per week

5. More than 3 times per week

HOW MANY HOURS PER DAY DO YOU EXERCISE?

1. Less than 30 minutes per day

2. 30 to 40 minutes per day

3. 50 minutes to 1 hour per day

4. 1 to 2 hours per day

5. More than 2 hours per day

WHAT PHYSICAL ACTIVITIES DO YOU USUALLY DO

TO EXERCISE?

(You can choose more than one answer)

1. Weight training

2. Walking

3. Jogging

5. Being part of a sports team

6. Dancing

7. Others.
WHAT IS THE EXTEND OF THE IMPORTANCE OF EXERCISE TO YOUR HEALTH?

1. Extremely important

2. Very important

3. Fairly important

4. Not important at all

WHAT IS THE IMPORTANCE OF DOING EXERCISE FOR HEMODIALYSIS PATIENTS?

1. Improves blood pressure

2. Removal of toxins from the blood

3. Improves blood glucose level

4. Improves blood lipid profile

5. Other

DO YOU CONSIDER EXERCISING SAFE FOR

HEMODIALYSIS PATIENTS

1. Yes, it is safe

2. No, it is not safe

WHEN CAN YOU START EXERCISING?

1. Never

2. I don't know

3. Anytime

4. After consulting the doctor

WHAT TYPE OF EXERCISE IS CONSIDERED SAFE?

(You can choose more than one answer)

1. Jogging

2. Swimming

3. High Jump

4. Wall Climbing

5. Weight lifting

6. Biking

7. Pressure training 

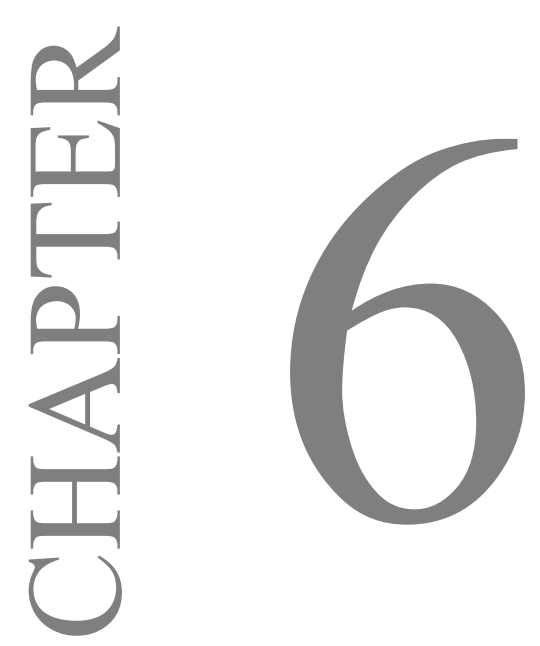

MALNUTRITION INFLAMMATION SCORE IS

BETTER DIAGNOSTIC TOOL THAN PHASE ANGLE WHEN COMPARED TO GLIM CRITERIA AMONG HEMODIALYSIS PATIENTS - RESULTS FROM A CROSS SECTIONAL STUDY

Karavetian M, Salhab N, Rizk R, Poulia L 


\begin{abstract}
Background and Aims: Malnutrition is prevalent in hemodialysis (HD) patients and is associated with increased risk of morbidity and mortality. The aim of this study was to explore the prevalence of malnutrition using the malnutrition inflammation score (MIS) and the bio-electrical impedance analysis (BIA) and to compare their concordance with the new Global Initiative on Malnutrition (GLIM) criteria for the diagnosis of malnutrition.
\end{abstract}

Methods: Seventy HD patients were assessed in a cross sectional study from a tertiary hospital in the United Arab Emirates. Malnutrition was diagnosed based on the GLIM criteria and the MIS questionnaire. We evaluated the agreement between the diagnostic following tools MIS, phase angle ( $\mathrm{PhA})$ derived from the BIA and GLIM. Finally, optimal gender specific cut-off points were identified for PhA according to the GLIM criteria.

Results: Almost half of the sample was diagnosed as malnourished according to MIS and GLIM criteria (54.29\%). A fair agreement was observed between the GLIM criteria with MIS $(\mathrm{k}=0.202)$ and $\mathrm{PhA}(\mathrm{k}=0.279)$, respectively among the malnourished patients. The PhA had better sensitivity but worse specificity compared to the MIS. The optimum cut-off point of $\mathrm{PhA}$ to detect malnutrition according to GLIM criteria was $\mathrm{PhA} \leq 5.7^{\circ}$ for males and $\leq 3.8^{\circ}$ for females.

Conclusion: The MIS tool performed slightly better than the $\mathrm{PhA}$ in the diagnosis of malnutrition among HD patients in the spectrum of the GLIM criteria.

Keywords: malnutrition, malnutrition inflammation score, phase angle, global initiative on malnutrition

Conflict of interest statement: The authors declare no conflict of interest. 


\section{INTRODUCTION}

Malnutrition in hemodialysis (HD) is a well-established condition, and often co-exists with inflammation (Kalantar-Zadeh et al., 2004). An estimated 50 to $75 \%$ of HD patients show signs of the malnutrition-inflammation complex syndrome (MICS) (Sabatino et al., 2016), depending on the diagnostic tool used. This syndrome is closely associated with adverse patient outcomes, such as frailty and depression, higher morbidity and mortality, and worsened quality of life (QoL) (Ikizler et al., 2013; Sabatino et al., 2016). The global outcome is a vicious cycle of the MICS and its consequences lead to chronicity (Sabatino et al., 2016). Accordingly, a careful and periodic nutritional screening, coupled with a timely diagnosis and a close monitoring of nutritional status are cornerstone for implementing tailored interventions for the prevention and treatment of the MICS (Sabatino et al., 2016). This necessitates the early identification of nutritional risk by the use of accurate and sensitive nutritional screening tools followed by nutritional assessment and diagnosis of malnutrition based on the specific diagnostic criteria. (Poulia et al., 2017, Cederholm et al, 2018).

Traditionally, tools employed to diagnose the MICS in HD patients ranged from anthropometry, to biochemical parameters, and numerous composite scores parallel to clinical judgment (Rogowski et al., 2018; Da Silva et al., 2018; Garcia et al., 2013). Among the latter, the Malnutrition-Inflammation Score (MIS) emerged as a comprehensive quantitative scoring system for diagnosing the MICS (Kalantar-Zadeh et al., 2001; KalantarZadeh et al., 2004; Pisetkul et al., 2010; Rambod et al., 2009). However, its use in clinical practice requires an interview with the patient, a thorough physical examination of subcutaneous body fat and signs of muscle wasting, in addition to biochemical variables including serum albumin level, total iron-binding capacity (TIBC) or transferrin level (Kalantar-Zadeh et al., 2001). Within the time constraints reported among renal dietitians (Wolfe, 2012), there is a pressing need for an accurate, easy-to-use and cost-effective diagnostic tool. In recent years, bioelectrical impedance analysis (BIA)-derived phase angle $(\mathrm{PhA})$ has gained attention in this regard, especially that the Academy of Nutrition and Dietetics stated that the use of BIA is appropriate in CKD patients (The American Dietetic Association, 2010). The $\mathrm{PhA}$ is proportional to the ratio of reactance and resistance between body cell mass and fat-free mass (Baumgartner et al., 1988), and is assumed to indicate cell integrity (Barbosa \& Barros, 2005). In various clinical fields, PhA was shown to be reliable marker for the detection of early malnutrition (Kyle et al., 2012; Wiech et al., 2018). 
The primary aim of this study was to explore the prevalence of malnutrition in a sample of HD patients in a hospital-based dialysis unit in the United Arab Emirates (UAE), using the MIS and the BIA and comparing them with the new Global Initiative on Malnutrition (GLIM) criteria for the diagnosis of malnutrition. The secondary aim was to find out the specific cut-off point of phase angle for the studied population, and the differences among the genders.

\section{MATERIALS AND METHODS}

\section{Study design}

This was a cross-sectional study conducted in September 2017, in a tertiary hospital-based HD unit in the United Arab Emirates (UAE). Ethical approval was obtained from Zayed University and Ministry of Health and Prevention (MOHP), and informed consent was obtained from all the patients. We took all eligible patients in the dialysis unit; the unit was randomly chosen and was one of the largest dialysis units in the country. There is no official renal registry to know what is the total number of HD patients, thus a power analysis could not be carried out. Eligible patients had to be stable adult patients on HD, free of acute diseases, not hospitalized in past 6 months and willing to participate in the study. Patients with heart pace maker, pregnant or not meeting the inclusion criteria were excluded. All eligible patients were invited to participate in the study. All demographic and clinical data were collected from electronic medical records.

\section{Malnutrition-inflammation score (MIS)}

The MIS is a validated tool frequently used for assessing the malnutrition-inflammation status of the patients. This is a composite score of ten components, each with four levels of severity, ranging from 0 (normal) to 3 (severely abnormal). The scoring sheet is composed of four sections: 1) patients' related medical history including weight change in edema-free post-HD body weight in the past 6 months, dietary intake, gastro-intestinal symptoms, functional capacity and comorbidities; 2) physical exam of subcutaneous body fat and signs of muscle wasting according to the Subjective Global Assessment (SGA) criteria; 3) Body Mass Index (BMI); and 4) laboratory parameters, i.e. serum albumin level and TIBC (Kalantar-Zadeh et al., 2001). The sum of all components ranges from 0 (normal) to 30 (severely abnormal). A higher score reflects more severe degree of malnutrition and inflammation. The assessment was performed by trained dietitians at the end of the HD session. 
In 2018, the Global Initiative on Malnutrition (GLIM) was introduced (Cederholm et al, 2018). GLIM is a two-step approach for the malnutrition diagnosis, incorporating as a first step the initial screening with any validated screening tool, in order to identify patients "at risk". The second step is the assessment for the diagnosis and last the grading for the severity of malnutrition. The assessment criteria for malnutrition included phenotypic criteria, which are: 1) non-volitional weight loss (weight loss of $>5 \%$ within the past 6 months), 2) low BMI $\left(<20 \mathrm{~kg} / \mathrm{m}^{2}\right.$ if $<70$ years, $<22 \mathrm{~kg} / \mathrm{m}^{2}$ if $\geq 70$ years), 3) reduced muscle mass (fat free mass index (FFMI) $<17 \mathrm{~kg} / \mathrm{m}^{2}$ for males and $<15 \mathrm{~kg} / \mathrm{m}^{2}$ for females) (Cederholm \& Jensen, 2015) and two etiologic criteria which are reduced food intake or assimilation and inflammation or disease burden. In order to diagnose malnutrition at least one phenotypic and one etiologic criterion should be present. For this study, we assessed the non-volitional weight-loss based on the previous European Society for Parenteral and Enteral Nutrition (ESPEN) recommendation, which is $>5 \%$ within the last 3 months (Cederholm \& Jensen, 2015). Furthermore, the phenotypic grading of malnutrition was described as Stage 1 (moderate) and Stage 2 (severe) malnutrition (Cederholm et al, 2018) using the sarcopenia cut-off points for low muscle quantity, where patients with a low appendicular muscle mass (ASM) $<20 \mathrm{~kg}$ in males and $<15 \mathrm{Kg}$ in females were classified as stage 2 malnourished patients. Higher or equal ASM values indicated Stage 1 malnutrition. ASM was derived from the skeletal muscle mass (SMM) measured by the BIA, and the cup-off points were taken from the consensus of the European Working Group on Sarcopenia in Older People 2 (EWGSOP2) (Cruz-Jentoft et al., 2018).

\section{Phase angle (PhA)}

The reaction of the body to an electric current, for instance when using a BIA, comprises 2 components: 1) resistance, which is the restriction to the electrical current flow due to extracellular water (ECW) and intra-cellular water (ICW); 2) reactance, which is the resistive effect of the body tissues and cell membranes. The $\mathrm{PhA}$ is a parameter that reflects the amount of reactance in the human body relative to the amount of resistance. The $\mathrm{PhA}$ is calculated based on the following formula: reactance/resistance*180/ $\pi$ (Baumgartner et al., 1988). PhA decreases with increased disease severity (Graf et al., 2018), and age (Gonzales et al., 2016). In the current study, BIA was performed using the SECA (mBCA 525- Germany) device in a supine position according to the ESPEN guidelines on BIA application (Kyle et al., 2004). The device takes 8 points into measurement: right arm, left arm, right leg, left leg, right half of body, left half of body, torso. Height was measured in the clinic, patients has 
voided bladder, were assessed within 10 minutes after HD session, without electrical interferences and according to all other manufacturer recommendations. The standardized $\mathrm{PhA}$ value, adjusted for the age and gender of the patient, was directly generated by the BIA.

\section{Statistical analysis}

Collected data were analyzed using the IBM SPSS Statistics 21.0 package. Normality of the data was checked using the Kolmogorov-Smirnov test. Normally distributed data for quantitative variables were presented as means and standard deviations, whereas skewed data were summarized as medians and interquartile range (IQR) values. Qualitative variables were expressed in frequencies. Gender-based and low $\mathrm{PhA}$-based differences for quantitative variables were analyzed using the Independent-samples T-test for normally distributed variables and Mann-Whitney $U$ non-parametric test for skewed variables; those for qualitative variables were analyzed using the Chi-square-test. The linear correlation between the MIS and PhA was investigated using the Pearson correlation coefficient. Concordance of the MIS and PhA were analyzed using the MedCalc software. The Receiver Operating Characteristic (ROC) curves (De Long et al., 1988) were also used to evaluate the ability of the MIS and $\mathrm{PhA}$ to correctly distinguish the well nourished from the malnourished as diagnosed by the GLIM criteria of malnutrition. Area under the ROC curve equal to 0.5 indicates that a tool cannot distinguish between the two groups, whereas area under the ROC curve equal to 1 indicates perfect separation of the values of the two groups. Furthermore, sensitivity, specificity, positive and negative predictive values, and positive and negative likelihood ratios were calculated. p-value $\leq 0.05$ was retained for statistical significance. Finally, Cohen's kappa ( $\kappa)$ was calculated to assess the agreement between MIS and GLIM, and $\mathrm{PhA}$ and GLIM in diagnosing malnutrition. $\kappa$ ranges from -1 to +1 . Based on the guidelines from Altman (1999), a $\kappa$ less than 0.2 represents a poor strength of agreement, $\kappa$ between 0.21 and $0.40,0.41$ and $0.60,0.61$ and 0.80 , and 0.81 and 1.00 represent a fair, moderate, good and very good agreement, respectively. All quartile splits for $\mathrm{PhA}$ were used because they are especially useful in summarizing skewed data. Finally, we used the positive predictive value (PPV), the negative predictive value (NPV) and the likelihood ratios to study the concordance of GLIM and MIS $(>10)$ and $\mathrm{PhA}\left(\leq 5.7^{\circ}\right)$. These measures were included as they are important in public health and health planning (Grunau \& Linn, 2018)

\section{RESULTS}

\section{Patients' Characteristics}


Seventy adult HD patients (Males: 61.4\%; Females: 38.6\%) were included in the study. Their average age was $54.61 \pm 12.79$ years, with males being older than females $(57.42 \pm 12.68$ vs. $50.15 \pm 11.85$ years, respectively, $\mathrm{p}=0.019)$. The mean BMI was $27.22 \pm 6.48 \mathrm{Kg} / \mathrm{m}^{2}$. Hypertension was the most common comorbidity $(88.57 \%)$, followed by diabetes $(65.71 \%)$ and cardiovascular diseases $(40.00 \%)$. Descriptive data of the sample is presented in Table 1.

Table 1: Characteristics of the sample of patients $(n=70)$

\begin{tabular}{|c|c|c|c|}
\hline & \begin{tabular}{|l|} 
Total \\
N (\%)
\end{tabular} & $\begin{array}{l}\text { Males } \\
\text { N (\%) }\end{array}$ & $\begin{array}{l}\text { Females } \\
\text { N (\%) }\end{array}$ \\
\hline Sample size $^{\mathrm{a}}$ & $70(100)$ & $43(61.4)$ & $27(38.6)$ \\
\hline Age (years) $)^{b}$ & $54.61 \pm 12.79$ & $57.42 \pm 12.68$ & $50.15 \pm 11.85$ \\
\hline BMI $\left(\mathrm{Kg} / \mathrm{m}^{2}\right)^{\mathrm{b}}$ & $27.22 \pm 6.48$ & $26.82 \pm 5.12$ & $27.83 \pm 8.27$ \\
\hline $\begin{array}{l}\text { Comorbidities }^{\mathrm{q}} \\
\text { Diabetes }^{\mathrm{a}} \\
\text { Hypertension }^{\mathrm{a}} \\
\text { Cardiovascular Diseases }^{\mathrm{a}} \\
\text { Others }^{\mathrm{a}}\end{array}$ & $\begin{array}{l}46(65.71) \\
62(88.57) \\
28(40.00) \\
37(52.86)\end{array}$ & $\begin{array}{l}28(65.12) \\
36(83.72) \\
19(44.19) \\
18(41.87)\end{array}$ & $\begin{array}{l}18(66.67) \\
26(96.30) \\
9(33.33) \\
19(82.61)\end{array}$ \\
\hline $\begin{array}{l}\text { Dialysis Vintage } \\
<1 \text { year }^{\mathrm{a}} \\
1-4 \text { years }^{\mathrm{a}} \\
>4 \text { years }^{\mathrm{a}}\end{array}$ & $\begin{array}{l}5(7.14) \\
35(50.00) \\
30(42.86) \\
\end{array}$ & $\begin{array}{l}4(9.30) \\
19(44.19) \\
20(46.51)\end{array}$ & $\begin{array}{l}1(3.70) \\
16(59.26) \\
10(37.04)\end{array}$ \\
\hline Malnourished as per GLIM criteria ${ }^{\mathrm{a}}$ & $38(54.29)$ & $23(53.48)$ & $15(55.55)$ \\
\hline Stage $1^{\text {a* }}$ & $12(31.58)$ & $6(26.08)$ & $6(40)$ \\
\hline Stage $2^{\mathrm{a} * \#}$ & $26(68.42)$ & $17(73.91)$ & $9(60)$ \\
\hline Malnourished as per MIS ${ }^{\mathrm{a}}$ & $34(48.57)$ & $16(37.21)$ & $18(66.66)$ \\
\hline
\end{tabular}

a Values expressed as frequencies and percentages; b Values expressed as mean and standard deviation; $\uparrow$ Percentages do not sum up due to multiple answers; *Severity grading is based upon the existence of 1 phenotypic criterion, which is reduced muscle mass in this study; $¥$ Severity grading of malnutrition: stage 1 (moderate) and stage 2 (severe) malnutrition

\section{Study Variables}

Study variables of the full sample and for each gender are presented in Table 2. The mean MIS of the participants was $9.40 \pm 3.07$, with no statistically significant difference between males and females $(9.00 \pm 3.15$ vs. $10.03 \pm 2.88$, respectively). SMM was seen to be within the normal values for both genders, but females showed to have significantly lower muscle mass then males (15.87 \pm 4.19 . versus 22.06 \pm 6.48$)$. Fat mass index (FMI) also showed a significant difference between the 2 genders where females had higher fat mass then males (12.01 \pm 6.08 versus $8.98 \pm 3.84)$. Only $2.9 \%$ of the sample had low FMI $\left(<3.8 \mathrm{Kg} / \mathrm{m}^{2}\right.$ in female and $<1.2 \mathrm{Kg} / \mathrm{m}^{2}$ in male) without differences between men and women $(2.3 \% \mathrm{vs} .3 .7 \%$, respectively). Moreover, the FFMI also showed that females had significantly less muscle 
mass then males, but the values did not reach to malnutrition (15.08 \pm 2.74 versus $17.90 \pm 3.44$ respectively).

Table 2. Study variables in all patients based on gender $(\mathbf{n}=\mathbf{7 0})$

\begin{tabular}{|l|l|l|l|l|}
\hline & Total & Males & Females & P value \\
\hline & Mean \pm SD & Mean \pm SD & Mean \pm SD & \\
\hline Age (years) & $54.61 \pm 12.79$ & $57.42 \pm 12.68$ & $50.15 \pm 11.85$ & $0.019^{*}$ \\
\hline $\begin{array}{l}\text { SMM (Kg) } \\
\text { (Malnourished: } \mathrm{M}<20 \mathrm{Kg} \& \mathrm{~F}<15 \mathrm{Kg})\end{array}$ & $19.67 \pm 6.44$ & $22.06 \pm 6.48$ & $15.87 \pm 4.19$ & $<0.001^{*}$ \\
\hline PhA $\left(^{\circ}\right)^{9}$ & $4.66 \pm 1.21$ & $4.64 \pm 1.24$ & $4.69 \pm 1.18$ & 0.857 \\
\hline MIS (malnourished $\geq 10)$ & $9.40 \pm 3.07$ & $9.00 \pm 3.15$ & $10.04 \pm 2.88$ & 0.171 \\
\hline $\begin{array}{l}\text { FMI }\left(\mathrm{Kg} / \mathrm{m}^{2}\right) \\
(\text { Malnourished: } \mathrm{M}<1.2 \mathrm{Kg} / \mathrm{m} 2 \& \mathrm{~F}<3.8 \mathrm{Kg} / \mathrm{m} 2)\end{array}$ & $10.15 \pm 5.00$ & $8.98 \pm 3.84$ & $12.01 \pm 6.08$ & 0.02 \\
\hline Fat $(\mathrm{Kg})$ & $26.70 \pm 12.57$ & $24.95 \pm 10.80$ & $29.49 \pm 14.77$ & 0.143 \\
\hline & Median $(\mathrm{IQR})$ & Median $(\mathrm{IQR})$ & Median $(\mathrm{IQR})$ & \\
\hline BMI $\left(\mathrm{Kg} / \mathrm{m}^{2}\right)$ & $25.71(7.35)$ & $25.82(5.38)$ & $25.00(10.54)$ & 0.986 \\
\hline $\begin{array}{l}\text { FFMI }\left(\mathrm{Kg} / \mathrm{m}^{2}\right) \\
(\text { Malnourished: }<17 \mathrm{Kg} \& \mathrm{~F}<15 \mathrm{Kg})\end{array}$ & $17.09(3.33)$ & $17.90(3.44)$ & $15.08(2.74)$ & $0.004^{*}$ \\
\hline FFM $(\mathrm{Kg})$ & $42.92(15.14)$ & $49.70(15.04)$ & $38.58(8.41)$ & $0.001^{*}$ \\
\hline TBW $(\mathrm{L})$ & $32.15(11.4)$ & $37.00(11.10)$ & $28.90(6.40)$ & $<0.001^{*}$ \\
\hline ECW $(\mathrm{L})$ & $15.20(4.7)$ & $16.20(4.70)$ & $13.30(3.90)$ & $0.001^{*}$ \\
\hline
\end{tabular}

Abbreviations: SMM: skeletal muscle mass; M: males; F: females; PhA: phase angle; MIS: malnutrition-inflammation score; FMI: fat mass index; BMI: body mass index; FFMI: fat free mass index; FFM: free fat mass; TBW: total body water; ECW: extracellular water

ๆPhA was analyzed for 69 patient; *significance at $p \leq 0.05$

Based on a cutoff of MIS $\geq 10$, almost half of the sample (48.57\%) suffered from MICS; which was more common among females $(66.7 \%$ vs. $37.2 \%$ in males, $\mathrm{p}=0.016)$. As for the GLIM criteria, $54.29 \%$ of the patients were classified as malnourished, and most of them $(68.42 \%)$ were in Stage 2; with no significant difference between the genders (males: $55.5 \%$ vs. females: $53.4 \%$. $\mathrm{p}=0.866$ )

The mean of the BIA-derived $\mathrm{PhA}$ of the participants was $4.66^{\circ} \pm 1.21^{\circ}$, with no genderbased difference $\left(4.64^{\circ} \pm 1.24^{\circ}\right.$ vs. $4.69^{\circ} \pm 1.18^{\circ}$ for males and females, respectively, $\mathrm{p}=0.857$ ). Quartile splits were almost similar between genders (In females: Q1 $=3.70^{\circ}, \mathrm{Q} 2=$ $4.70^{\circ}, \mathrm{Q} 3=5.80^{\circ}, \mathrm{IQR}=2.1^{\circ}$; In males: $\mathrm{Q} 1=3.45^{\circ}, \mathrm{Q} 2=4.75^{\circ}, \mathrm{Q} 3=5.55^{\circ}, \mathrm{IQR}=2.1^{\circ}$ ). On average, participants were at the $9.00 \pm 21.38^{\text {th }}$ reference $\mathrm{PhA}$ percentile, with females being at a higher reference percentile compared with males (14.37 \pm 25.06 vs. $5.55 \pm 18.10)$, $\mathrm{p}=0.044)$. PhA was analyzed for 69 patients due to 1 missing patient data. 


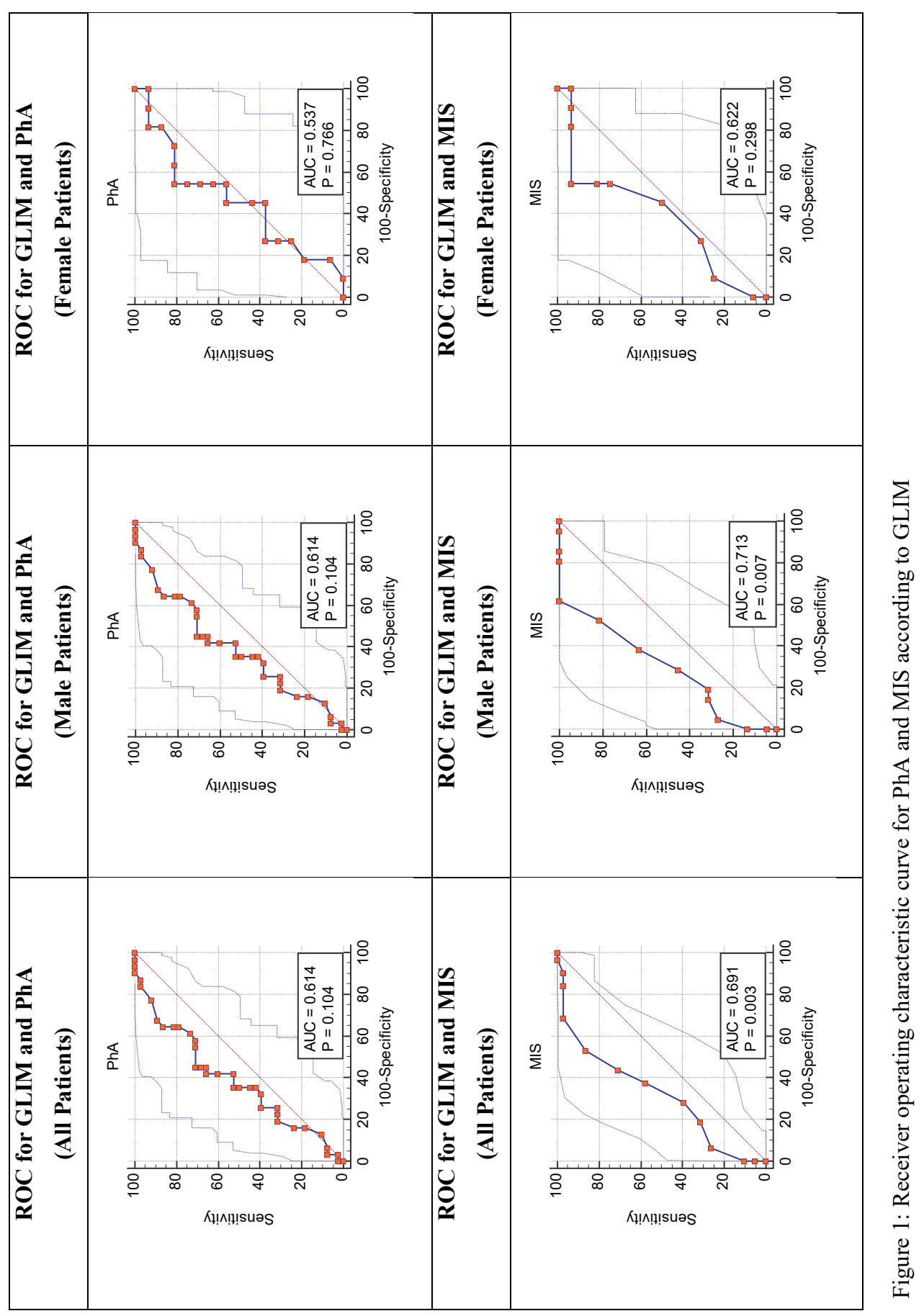




\section{Strength of Agreement between GLIM vs MIS and GLIM vs PhA}

To assess the agreement between the old and new malnutrition assessors Cohen Kappa ( $\kappa)$ was used to compare 1) a newly established malnutrition criteria (GLIM criteria) with an already established tool (MIS); and 2) GLIM with a newly emerging criteria (PhA).

According to the Cohen kappa statistics, the strength of agreement between MIS and GLIM criteria in diagnosing malnutrition was fair and not significant $(\kappa=-0.202$; $\mathrm{p}=0.089)$. The agreement was found to be slightly stronger in females $(\mathrm{k}=0.211$, $\mathrm{p}=0.268)$ than in males $(\mathrm{k}=0.168, \mathrm{p}=0.252)$.

According to the Cohen kappa statistics, the strength of agreement between $\mathrm{PhA}$ and in diagnosing malnutrition was fair and not significant $(\mathrm{k}=0.234, \mathrm{p}=0.029)$. The agreement was found to be stronger in males $(k=0.422, p=0.016)$ than in females $(\mathrm{k}=0.279, \mathrm{p}=0.135)$.

\section{Concordance of GLIM with MIS and PhA}

Further analysis was done to detect which of these assessors predicts malnutrition best, based on the GLIM criteria. Thus the Area under the curve (AUC) based on ROC statistics and the positive and negative likelihood ratios (LR+ and LR-, respectively) were calculated and presented in Table 3 . The identified cut-off point of $\mathrm{PhA}$ according to GLIM for the whole sample was $\mathrm{PhA} \leq 5.7^{\circ}$ (males: $\leq 5.7^{\circ}$, females: $\leq 3.8^{\circ}$ ); as for the MIS, the cut-off point of MIS $>10$ retrieved from the literature was used (Kalantar-Zadeh et al., 2001).

According to the results, the MIS seems to be slightly better correlated with the GLIM criteria according to the AUC, compared with the PhA ( $\mathrm{AUC}_{\mathrm{MIS}}=0.691, \mathrm{p}=0.003$, vs. $\left.\mathrm{AUC}_{\mathrm{PhA}}=0.614, \mathrm{P}=0.104\right)$. $\mathrm{PhA}$ was found to have a better sensitivity $(86.84 \%)$ compared with MIS (39.47\%), but a worse specificity (35.48 vs. 71.87, respectively). The MIS was found to have better LR+ and LR at the same time. 
Table 3. Concordance of GLIM with MIS $(>10)$ and $\operatorname{PhA}\left(\leq 5.7^{\circ}\right)$

\begin{tabular}{|l|l|l|}
\hline Criterion & MIS $>10$ & $\mathrm{PhA} \leq 5.7^{\circ}$ \\
\hline Sensitivity (\%) & 39.47 & 86.84 \\
\hline Specificity (\%) & 71.87 & 35.48 \\
\hline Positive predictive value (\%) & 50.0 & 62.63 \\
\hline Negative predictive value (\%) & 62.5 & 68.68 \\
\hline Positive likelihood ratio (LR+) & 1.40 & 1.35 \\
\hline Negative likelihood ratio (LR-) & 0.84 & 0.37 \\
\hline K value (p) & $0.202(0.089)$ & $0.234(0.029)$ \\
\hline AUC & 0.691 & 0.614 \\
\hline
\end{tabular}

\section{DISCUSSION}

To our knowledge, this is the first study that identifies the prevalence of malnutrition among HD patients in the UAE, while assessing the concordance of the BIA-derived $\mathrm{PhA}$ (a holistic assessment tool) and the MIS (an established assessment tool) with the GLIM diagnostic criteria for malnutrition. MIS had a better correlation than $\mathrm{PhA}$ with the GLIM criteria in identifying malnourished patients.

This study identified almost half of the HD patients as malnourished using the MIS. These finding were similar to the ability of GLIM to identify general malnourishment among our patients (54.29\%) thus showing a concordance between the 2 assessors. Alarmingly, $68.42 \%$ of the patients who fulfilled the GLIM criteria were categorized at stage 2 for malnutrition, i.e. severe malnutrition.

In the current study, there was a fair agreement for both assessors with the GLIM criteria; this could be attributed to the small sample size. Furthermore, the strength of agreement between GLIM and $\mathrm{PhA}$ was stronger in males than females; on the other hand, the strength of agreement between GLIM and MIS was stronger in females than males. This highlights the fact that females have a different specificity then men, and gender difference should be considered. This observation was previously reported in a study by da Silva et al. (2018), where the diagnostic accuracy using other parameters had low to moderate accuracy in men and low accuracy in women. Moreover, as in GLIM criteria muscle mass is assessed with objective measures rather than the subjective assessment in the MIS tool, may result in a more accurate and more valid diagnosis of malnutrition compared to other formally used tools of assessment of nutritional status. When identifying variables that differentiated the sample, gender was detected to be the factor that makes a significant difference in most studied 
parameters. Thus, a further analysis was conducted to identify the specific cut-off points between males and females. The specificity and sensitivity of PhA to GLIM was seen to be optimal at $5.7^{\circ}$ for males, and $3.8^{\circ}$ for females. These numbers might be considered as possible cut-off points to screen HD patients for malnutrition. In another setting, different cut-off points for $\mathrm{PhA}$ appeared to be a useful in screening nutritional status among males $\left(5.0^{\circ}\right)$ and females (4.6 $6^{\circ}$ (Kyle et al., 2012). Moreover, our results were in line with the findings of Bansal et al. (2018), where they demonstrated a $\mathrm{PhA}<5.59^{\circ}$ among chronic kidney disease (CKD) patients to be associated with mortality.

Contrary to the previous literature, there was no significant difference in $\mathrm{PhA}$ results between males and females which could be due to the small sample size of the females and to the low number of males with low muscle mass (sarcopenic) (Buffa et al., 2013; Slee et al., 2015; Jha et al., 2006). Since low muscle mass is associated with higher mortality (Johanson \& Lee, 2015), it is only wise to encourage physical exercise in HD patients to prolong longevity and increase QoL.

Based on our analysis, PhA had higher sensitivity than MIS using the GLIM criteria as a reference standard. This means that $\mathrm{PhA}$ can lead to a greater number of diagnosis of malnourished cases when compared with MIS. In fact, metabolic changes in cell membranes are first affected by malnutrition (Barbosa, 2008). As PhA may reflect cell integrity, it could detect malnutrition at an early stage. Unfortunately, when MISC develops in the patient, it forms a vicious cycle that is difficult to treat; thus measuring the PhA may be a tool to prevent MISC from being established.

In addition, the AUC suggested that clinically, MIS achieved slightly better than $\mathrm{PhA}$ in identifying malnutrition in HD patients, thus, confirming better performance. However, statistically, only MIS results were significant. The difference between the AUC of the MIS and PhA with GLIM was $<0.08$, denoting almost similar diagnostic capabilities when it comes to the whole sample. However, because the p value was significant for MIS, we concluded that MIS should at this stage be used as a better diagnostic tool than PhA.This was expected since MIS is a tool especially designed for this group of patients. In fact, a recent review reported that $\mathrm{PhA}$ may not be an accurate indicator of malnutrition. (Rinaldi et al., 2019). However, more research is needed on $\mathrm{PhA}$ accuracy, appropriateness, and cut-off points, particularly in HD patients. 
There are few limitations to this study, such as the sample was not powered to detect the prevalence of malnutrition and the use of a convenience selection sampling technique. These limitations do not allow us to generalize our findings to the whole HD population in the UAE. Finally, the assessments were based on a single measurement and this might have influenced the results, knowing the nature of CKD and the health fluctuations that affect HD patients.

The overall results of this study can lead us to speculate that the clinical difference between $\mathrm{PhA}$ and MIS are not very far apart, and that both have their strengths and weaknesses but do not result in major differences for the clinician and the patient. Consequently, the current research team invites future studies to look into larger samples for this patient subgroup and advocates clinicians to use the tool that is feasible for them, as long as it can fit within routine practice and resources available and results in routine identification of malnutrition among HD patients. In addition, larger studies are needed to validate the clinical effectiveness of using $\mathrm{PhA}$ as a practical tool for malnutrition screening.

In conclusion, malnutrition is widespread in the $\mathrm{HD}$ population in the UAE. Clinically, MIS performed slightly better than $\mathrm{PhA}$ in the diagnosis of malnutrition when using GLIM as a reference. Prioritizing malnutrition screening in this population, and integrating cost-effective, sensitive and specific tools within routine practice is advocated through the findings of this study. 


\section{REFERENCES}

Altman, D. G. (1999). Practical statistics for medical research. New York, NY: Chapman \& Hall/CRC Press.

Bansal, N., Zelnick, L. R., Himmelfarb, J., \& Chertow, G. M. (2018). Bioelectrical Impedance Analysis Measures and Clinical Outcomes in CKD. Am J Kidney Dis, 72(5), 662-672. doi:10.1053/j.ajkd.2018.03.030

Barbosa-Silva, MCG (2008|). Subjective and Objective Nutritional assessment methods: what do they really assess? Curr Opin Clin Nutr Metab Care, 11(3), 248-54.

Barbosa-Silva, M. C., \& Barros, A. J. (2005). Bioelectrical impedance analysis in clinical practice: a new perspective on its use beyond body composition equations. Curr Opin Clin Nutr Metab Care, 8(3), 311-317.

Baumgartner, R. N., Chumlea, W. C., \& Roche, A. F. (1988). Bioelectric impedance phase angle and body composition. Am J Clin Nutr, 48(1), 16-23. doi:10.1093/ajen/48.1.16

Buffa, R., Saragat, B., Cabras, S., Rinaldi, A. C., \& Marini, E. (2013). Accuracy of specific BIVA for the assessment of body composition in the United States population. PLoS One, 8(3), e58533. doi:10.1371/journal.pone.0058533

Cederholm, T., Bosaeus, I., Barazzoni, R., Bauer, J., Van Gossum, A., Klek, S., . . . Singer, P. (2015). Diagnostic criteria for malnutrition - An ESPEN Consensus Statement. Clin Nutr, 34(3), 335-340. doi:10.1016/j.clnu.2015.03.001

Cederholm, T., Jensen, G. L., Correia, M., Gonzalez, M. C., Fukushima, R., Higashiguchi, T., . . . Compher, C. (2018). GLIM criteria for the diagnosis of malnutrition - A consensus report from the global clinical nutrition community. Clin Nutr. doi:10.1016/j.clnu.2018.08.002

Cruz-Jentoft, A. J., Bahat, G., Bauer, J., Boirie, Y., Bruyere, O., Cederholm, T., . . . Zamboni, M. (2018). Sarcopenia: revised European consensus on definition and diagnosis. Age Ageing. doi:10.1093/ageing/afy169

da Silva, A. T., Hauschild, D. B., Moreno, Y. M. F., Bastos, J. L. D., \& Wazlawik, E. (2018). Diagnostic Accuracy of Bioelectrical Impedance Analysis Parameters 
for the Evaluation of Malnutrition in Patients Receiving Hemodialysis. Nutr Clin Pract. doi:10.1002/ncp.10098

DeLong, E., DeLong, D., \& Clarke-Pearson, D. (1988). Comparing the Areas under Two or More Correlated Receiver Operating Characteristic Curves: A Nonparametric Approach. Biometrics,44(3), 837-845. doi:10.2307/2531595

Garcia, M.F., Wazlawik, E., Franco Moreno, Y.M., Fhur, L.M, Gonzalez-Chica, D.A. (2013). Diagnostic accuracy of handgrip strength in the assessment of malnutrition in hemodialyzed patients. e-SPEN Journal , 8( 4), (e181 e186). doi.org/10.1016/j.clnme.2013.06.003

Gonzalez, M. C., Barbosa-Silva, T. G., Bielemann, R. M., Gallagher, D., \& Heymsfield, S. B. (2016). Phase angle and its determinants in healthy subjects: influence of body composition. Am J Clin Nutr, 103(3), 712-716. doi:10.3945/ajen.115.116772

Graf, C. E., Herrmann, F. R., \& Genton, L. (2018). Relation of Disease with Standardized Phase Angle among Older Patients. J Nutr Health Aging, 22(5), 601-607. doi:10.1007/s12603-018-1034-4

Grunau, G., \& Linn, S. (2018). Commentary: Sensitivity, specificity and predictive values: foundations, pliabilities, and pitfalls in research and practice. Front Public Health, 6, 256. doi:10.3389/fpubh.2018.00256

Ikizler, T. A., Cano, N. J., Franch, H., Fouque, D., Himmelfarb, J., Kalantar-Zadeh, K., . . . Wanner, C. (2013). Prevention and treatment of protein energy wasting in chronic kidney disease patients: a consensus statement by the International Society of Renal Nutrition and Metabolism. Kidney Int, 84(6), 1096-1107. doi:10.1038/ki.2013.147

Jha, V., Jairam, A., Sharma, M. C., Sakhuja, V., Piccoli, A., \& Parthasarathy, S. (2006). Body composition analysis with bioelectric impedance in adult Indians with ESRD: comparison with healthy population. Kidney Int, 69(9), 16491653. doi:10.1038/sj.ki.5000293

Johansen, K. L., \& Lee, C. (2015). Body composition in chronic kidney disease. Curr Opin Nephrol Hypertens, 24(3), 268-275. doi: $10.1097 / \mathrm{mnh} .0000000000000120$

Kalantar-Zadeh, K., Kopple, J. D., Block, G., \& Humphreys, M. H. (2001). A malnutrition-inflammation score is correlated with morbidity and mortality in 
maintenance hemodialysis patients. Am J Kidney Dis, 38(6), 1251-1263.

doi:10.1053/ajkd.2001.29222

Kalantar-Zadeh, K., Kopple, J. D., Humphreys, M. H., \& Block, G. (2004).

Comparing outcome predictability of markers of malnutrition-inflammation complex syndrome in haemodialysis patients. Nephrol Dial Transplant, 19(6), 1507-1519. doi:10.1093/ndt/gfh143

Kyle, U. G., Bosaeus, I., De Lorenzo, A. D., Deurenberg, P., Elia, M., Manuel Gomez, J., . . Pichard, C. (2004). Bioelectrical impedance analysis-part II: utilization in clinical practice. Clin Nutr, 23(6), 1430-1453. doi:10.1016/j.clnu.2004.09.012

Kyle, U. G., Soundar, E. P., Genton, L., \& Pichard, C. (2012). Can phase angle determined by bioelectrical impedance analysis assess nutritional risk? A comparison between healthy and hospitalized subjects. Clin Nutr, 31(6), 875881. doi:10.1016/j.clnu.2012.04.002

Pisetkul, C., Chanchairujira, K., Chotipanvittayakul, N., Ong-Ajyooth, L., \& Chanchairujira, T. (2010). Malnutrition-inflammation score associated with atherosclerosis, inflammation and short-term outcome in hemodialysis patients. J Med Assoc Thai, 93 Suppl 1, S147-156.

Poulia, K. A., Klek, S., Doundoulakis, I., Bouras, E., Karayiannis, D., Baschali, A., . . . Chourdakis, M. (2017). The two most popular malnutrition screening tools in the light of the new ESPEN consensus definition of the diagnostic criteria for malnutrition. Clin Nutr, 36(4), 1130-1135. doi:10.1016/j.clnu.2016.07.014

Rambod, M., Bross, R., Zitterkoph, J., Benner, D., Pithia, J., Colman, S., . . . Kalantar-Zadeh, K. (2009). Association of Malnutrition-Inflammation Score with quality of life and mortality in hemodialysis patients: a 5-year prospective cohort study. Am J Kidney Dis, 53(2), 298-309. doi:10.1053/j.ajkd.2008.09.018

Rinaldi, S., Gilliland, J., O'Connor, C., Chesworth, B., \& Madill, J. (2019). Is phase angle an appropriate indicator of malnutrition in different disease states? A systematic review. Clin Nutr ESPEN, 29, 1-14. doi:10.1016/j.clnesp.2018.10.010

Rogowski, L., Kusztal, M., Golebiowski, T., Bulinska, K., Zembron-Lacny, A., Wyka, J., . . Dziubek, W. (2018). Nutritional assessment of patients with 
end-stage renal disease using the MNA scale. Adv Clin Exp Med, 27(8), 11171123. doi:10.17219/acem/75604

Sabatino, A., Regolisti, G., Karupaiah, T., Sahathevan, S., Sadu Singh, B. K., Khor, B. H., . . Fiaccadori, E. (2017). Protein-energy wasting and nutritional supplementation in patients with end-stage renal disease on hemodialysis. Clin Nutr, 36(3), 663-671. doi:10.1016/j.clnu.2016.06.007

Slee, A., Birc, D., \& Stokoe, D. (2015). Bioelectrical impedance vector analysis, phase-angle assessment and relationship with malnutrition risk in a cohort of frail older hospital patients in the United Kingdom. Nutrition, 31(1), 132-137. doi:10.1016/j.nut.2014.06.002

The American Dietetic Association. Chronic Kidney Disease. Evidence-based nutrition practice guideline. Chicago, IL, 2010.

Wiech, P., Dabrowski, M., Bazalinski, D., Salacinska, I., Korczowski, B., \& Binkowska-Bury, M. (2018). Bioelectrical Impedance Phase Angle as an Indicator of Malnutrition in Hospitalized Children with Diagnosed Inflammatory Bowel Diseases-A Case Control Study. Nutrients, 10(4). doi:10.3390/nu10040499

Wolfe, W. A. (2012). Moving the issue of renal dietitian staffing forward. J Ren Nutr, 22(5), 515-520. doi:10.1053/j.jrn.2012.04.006 

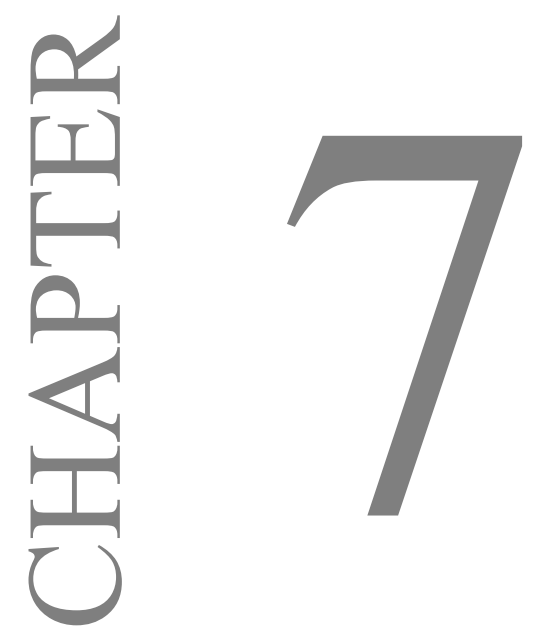

GENERAL DISCUSSION 


\section{GENERAL DISCUSSION}

The aim of this thesis was to study the effects of intradialytic aerobic exercise (IDE) on various clinical outcomes, barriers to physical activity (PA), and quality of life in hemodialysis (HD) patients in the United Arab Emirates (UAE). Furthermore, to provide further knowledge on the diagnosis of malnutrition using the malnutrition inflammation score (MIS) and the bioelectrical impedance analysis (BIA) derived phase angle $(\mathrm{PhA})$ by comparing them based on the Global initiative on malnutrition (GLIM) criteria.

\section{Chapter 2: Meta-analysis and determinants of an effective aerobic IDE program}

Regarding the effects of IDE, we first conducted a systematic review and metaanalysis on the effects of aerobic IDE, also to design the best protocol for our intervention based on the available literature. Previous research has shown the positive effect of IDE on HD patients, whether in improving dialysis efficiency (Sheng et al., 2014; Cheema \& Singh, 2005), quality of life (QOL) (Sheng et al., 2014; Cheema \& Singh, 2005; Chung et al., 2017; Smart \& Steele, 2011), peak oxygen consumption (Sheng et al., 2014; Cheema \& Singh, 2005; Chung et al., 2017), or solute clearance, notably serum phosphorus (P) (de Lima et al., 2013; Makhlough et al., 2012; Reboredo et al., 2010; Musavian et al., 2015). Despite these positive results retrieved from the literature, we aimed for a meta-analysis to have firmer conclusions to base our protocol on, especially that the sample size in most studies was small.

Regarding the meta-analysis, Pubmed, Medline (Ovid), Embase (Ovid), Cochrane, and Cinahl (EBSCO) databases were searched for all published interventions identifying the effects of aerobic IDE on QOL, dialysis efficiency, $\mathrm{P}$, vitamin $\mathrm{D}_{3}$, parathyroid hormone (PTH), C- reactive protein (CRP), intake of P binders, hospitalization rate, cost effectiveness, and mortality rate. Twenty-two articles met the inclusion criteria, and were included in the qualitative synthesis; only 12 articles met the criteria for the quantitative analysis. All of the studies, except for one (Makhlough et al., 2012), had cycling as the main exercise. The meta-analysis of five of the studies (Sakkas et al., 2008; Painter et al., 2000; Giannaki et al., 2013; Koh et al., 2010; Dobsak et al., 2012) showed that aerobic IDE had a significant positive effect on QOL- physical component score and QOL - mental component score. On the other hand, the meta-analysis of 3 (Wilund et al., 2010; Makhlough et al., 2012; De Lima et 
al., 2013) and 5 (Dobsak et al., 2012; Groussards et al., 2015; Parsons et al., 2004; Afshar et al., 2010; Sakkas et al., 2008) of the studies consecutively showed that IDE had no significant effect neither on serum $\mathrm{P}$, nor on dialysis efficiency $(\mathrm{Kt} / \mathrm{V})$. Nevertheless, we cannot underestimate the limitations in the available literature, ranging from the selection of the healthier HD patients, to the limited number of the available IDE trials, to the diversity in the exercise protocols that led to the small number of eligible interventions for meta-analysis. Finally, based on the included studies that showed statistically significant positive effects of IDE on outcomes parameters, this thesis suggests a putative recipe for aerobic exercise in chronic HD patients (Box 1).

\begin{tabular}{|ll|}
\hline $\begin{array}{ll}\text { Box 1: Putative recipe for aerobic IDE } \\
\text { Preconditioning } & \begin{array}{l}\text { Strength training and stretching exercises } \\
\text { for } 2 \text { months prior to exercise initiation }\end{array} \\
\text { Modality } & \text { Warm up: Range capacity exercise } \\
& + \text { Cycling } \\
\text { Frequency } & 3 \text { times per week } \\
\text { Intensity } & \text { Borg scale average rating of } 12 \text { points } \\
& \text { Or } 60-65 \% \text { of the peak power output } \\
\text { Duration } & \text { Range capacity exercises: } 15 \text { minutes } \\
& + \text { Cycling: } 30 \text { - } 45 \text { minutes }\end{array}$ \\
\hline
\end{tabular}

Despite the overall positive evidence, IDE is not part of the routine treatment protocol of HD patients; is it the lack of insufficient evidence to convince the treatment team, or the lack of guidelines? In all cases, most of the proofs came from interventions conducted in developed Western countries. As we all know, ethnic differences and cultural habits might impact this type of intervention, whether from the difference in muscle and fat distribution perspective or from the social habits and lifestyle angle; so, it is important to get more insight on IDE in developing middle-eastern countries. Thus, this thesis pioneered in introducing IDE in the UAE, as well as in identifying potential barriers. 


\section{Chapter 3: Current status of HD patients in the UAE}

According to the findings of the conducted review (chapter 2), the protocol of the present study was prepared (detailed on chapter 3 ). In addition, this chapter presented descriptive data of the sample of patients that were eligible and consented to participate in the study. Our results revealed a young, educated, Arab population, with a normal mean body mass index (BMI). Controversially to the normal BMI, $80.4 \%$ of the patients were mildly malnourished. Despite the fact that almost all of the patients reported "walking" as their exercise, still $83 \%$ of the them had $\geq 2$ barriers to PA, with "fatigue on dialysis days" being the most reported barrier. In favor to our IDE program, $90 \%$ of the patients were independent in their routine daily self-care, and almost half did not have any problem in walking. To add-on, few patients experienced anxiety and depression. All this raised our hope in achieving high success rate in our intervention.

\section{Chapter 4: Effects of IDE on hyperphosphatemia, clinical outcomes, and QOL}

Hyperphosphatemia is widely recognized as a mortality risk factor in HD patients (Amman et al., 1999). There is a general agreement that dialysis is not enough to remove the entire phosphate load, and many patients inevitably fall in the hyperphosphatemic category. Especially, the Kidney Disease Improving Global Outcomes (KDIGO) guidelines (2017) recommend lowering the serum phosphorus (P) levels towards the range that is considered normal for healthy populations (Ketteler et al., 2017). Extensive research was and is being conducted to find potential solutions to alleviate its consequences and thus improve the QOL of HD patients. In this regard, literature is suggesting that IDE holds potential clinical and social improvements to the HD patients. For instance, patient education on renal nutrition proved to be cost-effective (Rizk et al. 2017), and was associated with improvement

in serum P (Karavetian \& Rizk, 2018). The next topic of the present thesis reported on the effects of IDE intervention on hyperphosphatemia, clinical outcomes and QOL. The results of this study may be clinically relevant, as they identified the hyperphosphatemic population as the one that will most benefit from such interventions. In addition, we found that the mean adherence rate to the 6 months IDE program was $57.5 \%$, with an exercise intensity of 11.3 on the Borg scale, almost reaching a moderate intensity level of exercise (Wong et al., 2017). Unfortunately, $53.4 \%$ of the patients engaged in $<60 \%$ of the exercise sessions, and thus were considered to have a low adherence to the program (Anding et al., 2015). This study 
showed that, in hyperphosphatemic patients, IDE decreased the serum P level with a change approaching significance. Thus, our assumption that IDE along with the proper education on PA could be beneficial for HD patients was right for hyperphosphatemic patients. We acknowledge the small sample size $(\mathrm{n}=15)$ as a plausible limitation that did not allow the results to reach significance.

Similar to other research (Musavian et al., 2015), dialysis efficiency failed to improve in our study. Compared to studies that showed positive significant difference in URR and Kt/V (Parker et al., 2015; Reboredo et al., 2010; Parsons et al., 2006), ours had a smaller sample size, lacked a preconditioning phase and had a shorter IDE session duration.

In our HD sample, it was surprising to see that there was no significant difference in the QOL-VAS, although we were experiencing positive vibes with the patients. The patients that were adherent to the program transmitted a great positive attitude towards IDE, and acknowledged the effect of this intervention on their QOL. However, we suspected that our choice of the EQ-5D-5L was not culturally appropriate as we initially thought. In fact, while assessing the patients, we faced a limitation with this scale. Patients showed strong religious beliefs and interpreted sickness as "fate" and "will of God"; when we asked the patients to rate their health on the VAS, many used the word "Thank God" which they translated to a high score on the scale. Unfortunately, our patients failed to objectively assign a score on the VAS. In fact, the influence of religious beliefs on the QOL assessment has been reported in other cultures too (Moraes et al., 2014).

To our advantage, and aside from the QOL, religion had a positive effect on our patients. During the month of Ramadan, a religious event where Muslims fast during the day and eat after sunset, the mean patient minutes of exercise was at its highest (166.5 minutes), and adherence to the program was higher than in other months $(62 \%)$.

There is no doubt that the strict renal diet could lead to malnutrition in HD patients. In the present study, we were unable to improve the prevalent malnutrition score, but the MIS remained stable. However, due the lack of a comparison group, we could not prove our assumption that aerobic IDE could slow down the normal deterioration of the nutritional status of HD patients over time (Karavetian et al., 2016).

We hope that our intervention paved the way to future research in this field, specifically in the UAE population. In fact, we are proud to say that our IDE protocol 
will be adopted in 2 dialysis units in the UAE starting 2019. This marks a great ending for our study, and a new beginning in the world of renal care in the UAE.

\section{Chapter 5: Effect of IDE on the barriers to PA and position of the medical team towards this adjunct therapy}

Could IDE attenuate sedentarism, a phenomenon which is associated with adverse outcomes in HD patients? (Ohare et al., 2003; Stack et al., 2005; Tentori et al., 2010). To answer this question, this thesis looked at different components, including the barriers to PA in addition to the exercise behavior, attitude, and knowledge in HD patients. To date, the literature agrees on specific common barriers to PA; these include "fatigue, lack of energy, comorbidities lack of time and lack of access" (Hannan \& Bronas, 2017). Nevertheless, few studies report on the effect of exercise on these barriers. Our result on the most identified barrier to PA "fatigue on dialysis days" was in line with the literature (Jhamb et al., 2016; Fiaccadori et al., 2014; Bossola et al., 2014; Delgado \& Johansen, 2012; Kontos et al., 2007). We had achievements in this perspective, in that this intervention was able to remove all barriers to PA for almost $15 \%$ of the sample.

Also, we aimed to evaluate exercise behavior, attitude, and knowledge in HD patients. The intervention led to a significant improvement in patient's knowledge about the benefits of exercise. Also, we witnessed a $30 \%$ increase in the number of patients having the correct knowledge about the safety of exercise. This gave us more confident about the importance and effectiveness of the provided education material. We had also postulated that the duration of our 6 month intervention would be enough to change the lifestyle of our patients. Surprisingly, the number of patients exercising at follow up dropped back to the same number recorded at baseline.

Still, we feel that our findings are promising, as this intervention was able to sustain the acquired knowledge about the benefits and safety of exercise, although not the exercise behavior per se.

Finally, this study was the first to unveil the attitude of the nephrologists and nurses towards PA in HD patients in the UAE. Despite the positive attitude of the medical team toward PA in HD patients, doctors in the UAE were still not prescribing it mainly because of fear for the risks, for which neither our study nor the meta-analysis shows firm arguments. Therefore, both HD patients and staff should be properly 
educated on the benefits of PA, and on the best ways to introduce it safely to their lifestyle for a better health and QOL.

\section{Chapter 6: What is the best tool for malnutrition assessment based on the Global Initiative on malnutrition (GLIM) criteria?}

Various tools are used for malnutrition screening in HD patients; these included the Malnutrition-Inflammation Score (MIS), which was specifically designed for HD patients. However, MIS is time consuming, and requires an interview with the patient, a physical examination, in addition to blood parameters (Kalantar-Zadeh et al., 2004). Thus, emerges the need to find an accurate, easy-to-use and cost-effective malnutrition diagnostic tool. In recent years, bioelectrical impedance analysis (BIA)derived phase angle $(\mathrm{PhA})$ is being studied as an alternative practical way for malnutrition screening.

Lately, the GLIM initiative has proposed a new approach to the diagnosis of malnutrition (Cederholm et al., 2018). This chapter focused on comparing the effectiveness of the MIS and the bioelectrical impedance (BIA) derived - phase angle $(\mathrm{PhA})$ in the diagnosis of malnutrition based on the GLIM criteria. As expected the MIS tool, which was designed for HD patients, performed slightly better than $\mathrm{PhA}$ in the diagnosis of malnutrition. Adequate diagnosis of malnutrition is of great significance, since malnutrition is often hidden or misdiagnosed in this population due to the prevalent sarcopenic obesity (Dierkes et al., 2018). Hence, a proper and unified malnutrition assessment tool should be routinely used to assess the nutritional status of this population, and treat accordingly.

Literature had shown $\mathrm{PhA}$ to be a promising marker for the detection of early malnutrition in various conditions (Kyle et al., 2012; Wiech et al., 2018); however set cut-off points are still missing for HD patients. Our research team was able to detect gender as the factor that makes significant difference among other parameters, and generated cut-off points accordingly. Thus, the specificity and sensitivity of $\mathrm{PhA}$ to GLIM was seen to be optimal at $5.7^{\circ}$ for male, and $3.8^{\circ}$ for female. These numbers could be considered as the cut off points to screen HD patients for malnutrition. These findings should be validated in future research, especially that our results suggested that the clinical difference in malnutrition screening between MIS and PhA are not very far apart. As malnutrition is widespread in the HD population, specifically in the UAE, we advise clinicians to use any user friendly, cost effective, available 
assessment tool in their HD unit to ensure routine malnutrition patient screening. However, larger studies are needed to validate the clinical effectiveness of using $\mathrm{PhA}$ as a cost effective, practical tool for malnutrition screening. Early detection of malnutrition is crucial; unfortunately, when HD patients have already developed in the malnutrition inflammation syndrome complex, it is harder to treat.

\section{Strengths of the study}

First, our study was adequately powered to answer the main research questions. Moreover, the primary investigator (PI) and the supervised trained research assistants collected the data. In addition, the study was at all times under the direct supervision of the PI and the medical team. Second, the study sample characteristics (gender distribution, primary cause of $\mathrm{HD}$, comorbidities, work status) were very similar to the general HD population in the UAE (AlSahow et al., 2016). Third, to our knowledge, this was the first study to introduce IDE in the UAE. This new concept was welcomed by $72 \%$ of eligible patients. It was interesting to see that the intervention was contagious, where non-eligible patients and new comers wanted to join the IDE group. Furthermore, when presenting our study at the Emirates Medical Association, EMA-N Nephrology Club Meeting, nephrologists from other hospitals were interested to implement this program for their patients.

\section{Limitations of the study}

We acknowledge that there were several limitations to our study. The major limitation of the study was the absence of a comparative control group; one-fifty HD patients were dialyzing at Al Qassimi hospital, but only 57 were eligible for IDE. To account for the drop out rate, we opted to adopt a 1-group intervention trial over a randomized control trial, and tried to overcome this limitation by measuring the change at 3 time points; in fact, we had a $26.8 \%$ attrition rate at the end of the study. Another limitation was the subjective tool used to measure the intensity of the IDE. Initially, the protocol defined exercise intensity to be at the lowest level of moderate exercise intensity $\left(40 \% \mathrm{VO}_{2} \mathrm{R}\right)$ (Garber et al., 2011). Unfortunately, when testing our methods, we noticed that most of the patients were on beta-blocker medication, which impeded the increase of the resting heart rate. Consequently, intensity was assessed using the perceived exertion rating (Borg Scale) (Center of Disease Control and Prevention). A further limitation was the young age of the patients, which was normal considering 
the strict inclusion criteria, but is not representative of the general HD population. Finally, because of limited available funds, this study was conducted in only one hospital in the UAE, therefore, we could not generalize our results to all HD patients in this region.

\section{Tools used}

We assessed the nutritional status of the patients using the "Malnutrition Inflammation Score" (MIS) adapted from Kalantar-Zadeh et al. (2004). We opted for the MIS questionnaire because it was specifically design for HD patients. We also assessed the QOL using QOL "EQ-5D-5L” questionnaire (The EuroQol Group, 1990). We thought that this format for QOL assessment would be the most convenient and culturally appropriate for this population. Next, we developed the "Exercise Behavior, Attitude and Knowledge questionnaire" to assess exercise in our sample from different perspective. As for the barriers to PA, we assessed them using the "Barriers to Physical Activity" questionnaire adapted from Fiaccadori et al., (2013); we removed the question on amputation from the adopted barriers questionnaire, as this was an exclusion criterion in our study. The last 2 questionnaires were back translated from English to Arabic and then back to English to insure accuracy in translation.

All the questionnaires were tried out on patients when testing our methods to be sure that all questions were clearly stated.

Finally, we used the Borg Scale of perceived exertion (Center of Disease Control and Prevention) to quantify the intensity of IDE.

All data on demographics and blood parameters were collected from patient's file.

\section{Implications for Future Practice}

Despite all the limitations of the current study, we pioneered in implementing an IDE program in the UAE, and today, we say that IDE could promise clinical improvement for HD patients.

Based on our experience, the proper logistics need to be implemented for a smooth introduction of IDE in the routine treatment of HD patients. Thus, we suggest that the exercise equipment need to be kept in the treatment room for easy access. In addition, the nursing staff should be involved in the supervision of an IDE program, since the 
nurse is already performing most of the required tasks to monitor the patients during exercise (pulse rate, blood pressure, cramps). Aside from the nurse, exercise coordinators should be recruited to assist and educate the patients. Last but not least, the unit nephrologist should endorse the IDE program; we experienced the importance of the patient-physician trust during our trial, and its impact on patients' behavior. With our results, we are confident to advise nephrologists to start their journey with IDE, along with the trialed education that proved to be effective.

\section{Implications for Future Research}

Future studies should focus on targeting a larger sample to see the real effect of the intervention. In addition, a large sample would enhance the chances of including more elderly patients for it to be representative of the HD population; furthermore, it would allow researchers to design randomized controlled trials to validate these observations. Finally, there is an urge to define the proper modality, intensity, and duration for IDE to be embedded in HD treatment protocol; thus future studies should use equipment (e.g. cycle pedaler) with set intensity levels. To all researchers in this field, the fate of IDE is in your hands.

\section{Conclusions}

In conclusion, this study met its objective of studying the effects of IDE in HD patients in the UAE. However, the small sample size might have hindered the real effect of the intervention. The main findings of the thesis are outlined below:

- Hyperphosphatemia and malnutrition are widespread in the selected sample from a tertiaty hospital in the UAE.

- Aerobic IDE for 45 minutes, 2-3 times per week, is safe for HD patients.

- Aerobic IDE could be beneficial in normalizing serum P, especially in hyperphosphatemic patients.

- Adherence level to this program in a multi-ethnic culture is around $50 \%$.

- Aerobic IDE had no consistent effect on QOL, and urea reduction ratio (URR).

- Malnutrition status remained stable throughout the study.

- Aerobic IDE and patient's counseling may help HD patients overcome their barriers to exercise. 
- Patient's counseling may be associated with an increase in knowledge towards the benefits and safety of exercise in HD patients.

- The nephrologist's concern about the risks of exercise is a major factor preventing IDE implementation.

- Based on the Global Initiative on Malnutrition (GLIM) criteria, the Malnutrition Inflammation Score (MIS) is the preferred malnutrition assessment tool in HD patients. 


\section{REFERENCES}

Afshar, R., Shegarfy, L., Shavandi, N., \& Sanavi, S. (2010). Effects of aerobic exercise and resistance training on lipid profiles and inflammation status in patients on maintenance hemodialysis. Indian J Nephrol, 20(4), 185-189. doi:10.4103/0971-4065.73442

AlSahow, A., AlRukhaimi, M., Wakeel, J.A., Al-Ghamdi, S.M.G., AlGhareeb, S., AlAli, F.,... GCC-DOPPS 5 Study Group (2016). Demographics and key clinical characteristics of hemodialysis patients from the gulf cooperation council countries enrolled in the dialysis outcomes and practice patters study phase 5 (2012-2015). Saudi J Kidney Dis Transpl. 27(6suppl 1), S12-S23.

Amann, K., Gross, M. L., London, G. M., \& Ritz, E. (1999). Hyperphosphataemia--a silent killer of patients with renal failure? Nephrol Dial Transplant, 14(9), 2085-2087.

Anding, K., Bar, T., Trojniak-Hennig, J., Kuchinke, S., Krause, R., Rost, J. M., \& Halle, M. (2015). A structured exercise programme during haemodialysis for patients with chronic kidney disease: clinical benefit and long-term adherence. BMJ Open, 5(8), e008709. doi:10.1136/bmjopen-2015-008709

Bossola, M., Pellu, V., Di Stasio, E., Tazza, L., Giungi, S., \& Nebiolo, P. E. (2014). Self-reported physical activity in patients on chronic hemodialysis: correlates and barriers. Blood Purif, 38(1), 24-29. doi:10.1159/000363599

Cederholm, T., Jensen, G. L., Correia, M., Gonzalez, M. C., Fukushima, R., Higashiguchi, T., . . Compher, C. (2018). GLIM criteria for the diagnosis of malnutrition - A consensus report from the global clinical nutrition community. Clin Nutr. doi:10.1016/j.clnu.2018.08.002

Centers for Disease Control and Prevention. Borg Rating of Perceived Exertion Scale. Retrieved from https://www.cdc.gov/physicalactivity/basics/measuring/exertion.htm. Accessed 17 September 2017.

Cheema, B. S., \& Singh, M. A. (2005). Exercise training in patients receiving maintenance hemodialysis: a systematic review of clinical trials. $\mathrm{Am} \mathrm{J}$ Nephrol, 25(4), 352-364. doi:10.1159/000087184 
Chung, Y. C., Yeh, M. L., \& Liu, Y. M. (2017). Effects of intradialytic exercise on the physical function, depression and quality of life for haemodialysis patients: a systematic review and meta-analysis of randomised controlled trials. J Clin Nurs, 26(13-14), 1801-1813. doi:10.1111/jocn.13514

de Lima, M. C., Cicotoste Cde, L., Cardoso Kda, S., Forgiarini, L. A., Jr., Monteiro, M. B., \& Dias, A. S. (2013). Effect of exercise performed during hemodialysis: strength versus aerobic. Ren Fail, 35(5), 697-704. doi:10.3109/0886022x.2013.780977

Delgado, C., \& Johansen, K. L. (2012). Barriers to exercise participation among dialysis patients. Nephrol Dial Transplant, 27(3), 1152-1157. doi:10.1093/ndt/gfr404

Dierkes, J., Dahl, H., Lervaag Welland, N., Sandnes, K., Saele, K., Sekse, I., \& Marti, H. P. (2018). High rates of central obesity and sarcopenia in CKD irrespective of renal replacement therapy - an observational cross-sectional study. BMC Nephrology, 19(1), 259. doi:10.1186/s12882-018-1055-6

Dobsak, P., Homolka, P., Svojanovsky, J., Reichertova, A., Soucek, M., Novakova, M., ... Siegelova, J. (2012). Intra-dialytic electrostimulation of leg extensors may improve exercise tolerance and quality of life in hemodialyzed patients. Artif Organs, 36(1), 71-78. doi:https://dx.doi.org/10.1111/j.15251594.2011.01302.x

EuroQol--a new facility for the measurement of health-related quality of life. (1990). Health Policy, 16(3), 199-208.

Fiaccadori, E., Sabatino, A., Schito, F., Angella, F., Malagoli, M., Tucci, M., . . . Regolisti, G. (2014). Barriers to physical activity in chronic hemodialysis patients: a single-center pilot study in an Italian dialysis facility. Kidney Blood Press Res, 39(2-3), 169-175. doi:10.1159/000355793

Giannaki, C. D., Sakkas, G. K., Karatzaferi, C., Hadjigeorgiou, G. M., Lavdas, E., Kyriakides, T., .. . Stefanidis, I. (2013). Effect of exercise training and dopamine agonists in patients with uremic restless legs syndrome: a six-month randomized, partially double-blind, placebo-controlled comparative study. BMC Nephrology, 14, 194. doi:https://dx.doi.org/10.1186/1471-2369-14-194

Groussard, C., Rouchon-Isnard, M., Coutard, C., Romain, F., Malarde, L., LemoineMorel, S., . . Boisseau, N. (2015). Beneficial effects of an intradialytic 
cycling training program in patients with end-stage kidney disease. Applied Physiology, Nutrition, \& Metabolism = Physiologie Appliquee, Nutrition et Metabolisme, 40(6), 550-556. doi:https://dx.doi.org/10.1139/apnm-2014-0357

Hannan, M., \& Bronas, U. G. (2017). Barriers to exercise for patients with renal disease: an integrative review. J Nephrol, 30(6), 729-741. doi:10.1007/s40620-017-0420-Z

Jhamb, M., McNulty, M. L., Ingalsbe, G., Childers, J. W., Schell, J., Conroy, M. B., . . . Dew, M. A. (2016). Knowledge, barriers and facilitators of exercise in dialysis patients: a qualitative study of patients, staff and nephrologists. $B M C$ Nephrology, 17(1), 192. doi:10.1186/s12882-016-0399-z

Kalantar-Zadeh, K., Kopple, J. D., Humphreys, M. H., \& Block, G. (2004). Comparing outcome predictability of markers of malnutrition-inflammation complex syndrome in haemodialysis patients. Nephrol Dial Transplant, 19(6), 1507-1519. doi:10.1093/ndt/gfh143

Karavetian, M., Elzein, H., Rizk, R., Jibai, R., \& de Vries, N. (2016). Nutritional education for management of osteodystrophy: Impact on serum phosphorus, quality of life, and malnutrition. Hemodial Int, 20(3), 432-440. doi:10.1111/hdi.12405

Karavetian, M., \& Rizk, R. (2018). Patient education for hyperphosphatemia management: Improving outcomes while decreasing costs? Kidney Res Clin Pract, 37(1), 4-7. doi:10.23876/j.krcp.2018.37.1.4

Ketteler M, Block GA, Evenepoel P, et al. Executive summary of the 2017 KDIGO Chronic Kidney Disease-Mineral and Bone Disorder (CKD-MBD) Guideline Update: what's changed and why it matters. Kidney Int. 2017;92:26-36. (2017). Kidney Int, 92(6), 1558. doi:10.1016/j.kint.2017.10.001

Kontos, P. C., Miller, K. L., Brooks, D., Jassal, S. V., Spanjevic, L., Devins, G. M., . . . Naglie, G. (2007). Factors influencing exercise participation by older adults requiring chronic hemodialysis: a qualitative study. Int Urol Nephrol, 39(4), 1303-1311. doi:10.1007/s11255-007-9265-z

Kyle, U. G., Soundar, E. P., Genton, L., \& Pichard, C. (2012). Can phase angle determined by bioelectrical impedance analysis assess nutritional risk? A comparison between healthy and hospitalized subjects. Clin Nutr, 31(6), 875881. doi:10.1016/j.clnu.2012.04.002 
Koh, K. P., Fassett, R. G., Sharman, J. E., Coombes, J. S., \& Williams, A. D. (2010). Effect of intradialytic versus home-based aerobic exercise training on physical function and vascular parameters in hemodialysis patients: a randomized pilot study. American Journal of Kidney Diseases, 55(1), 88-99. doi:10.1053/j.ajkd.2009.09.025

Makhlough, A., Ilali, E., Mohseni, R., \& Shahmohammadi, S. (2012). Effect of intradialytic aerobic exercise on serum electrolytes levels in hemodialysis patients. Iran J Kidney Dis, 6(2), 119-123.

Moraes, C., Marinho, S. M., da Nobrega, A. C., de Oliveira Bessa, B., Jacobson, L. V., Stockler-Pinto, M. B., . . Mafra, D. (2014). Resistance exercise: a strategy to attenuate inflammation and protein-energy wasting in hemodialysis patients? Int Urol Nephrol, 46(8), 1655-1662. doi:10.1007/s11255-014-0712-3

Musavian, A. S., Soleimani, A., Masoudi Alavi, N., Baseri, A., \& Savari, F. (2015). Comparing the effects of active and passive intradialytic pedaling exercises on dialysis efficacy, electrolytes, hemoglobin, hematocrit, blood pressure and health-related quality of life. Nurs Midwifery Stud, 4(1), e25922.

O'Hare, A. M., Tawney, K., Bacchetti, P., \& Johansen, K. L. (2003). Decreased survival among sedentary patients undergoing dialysis: results from the dialysis morbidity and mortality study wave 2. American Journal of Kidney Diseases, 41(2), 447-454. doi:10.1053/ajkd.2003.50055

Painter, P., Carlson, L., Carey, S., Paul, S. M., \& Myll, J. (2000). Physical functioning and health-related quality-of-life changes with exercise training in hemodialysis patients. American Journal of Kidney Diseases, 35(3), 482-492.

Parker, K., Zhang, X., Lewin, A., \& MacRae, J. M. (2015). The association between intradialytic exercise and hospital usage among hemodialysis patients. Applied Physiology, Nutrition, \& Metabolism = Physiologie Appliquee, Nutrition et Metabolisme, 40(4), 371-378. doi:10.1139/apnm-2014-0326

Parsons, T. L., Toffelmire, E. B., \& King-VanVlack, C. E. (2004). The effect of an exercise program during hemodialysis on dialysis efficacy, blood pressure and quality of life in end-stage renal disease (ESRD) patients. Clinical Nephrology, 61(4), 261-274. 
Parsons, T. L., Toffelmire, E. B., \& King-VanVlack, C. E. (2006). Exercise training during hemodialysis improves dialysis efficacy and physical performance. Arch Phys Med Rehabil, 87(5), 680-687. doi:10.1016/j.apmr.2005.12.044

Reboredo Mde, M., Henrique, D. M., Faria Rde, S., Chaoubah, A., Bastos, M. G., \& de Paula, R. B. (2010). Exercise training during hemodialysis reduces blood pressure and increases physical functioning and quality of life. Artif Organs, 34(7), 586-593. doi:10.1111/j.1525-1594.2009.00929.x

Rizk, R., Hiligsmann, M., Karavetian, M., \& Evers, S. (2017). Cost-effectiveness of dedicated dietitians for hyperphosphatemia management among hemodialysis patients in Lebanon: results from the Nutrition Education for Management of Osteodystrophy trial. J Med Econ, 20(10), 1024-1038. doi:10.1080/13696998.2017.1347877

Sakkas, G. K., Hadjigeorgiou, G. M., Karatzaferi, C., Maridaki, M. D., Giannaki, C. D., Mertens, P. R., . . . Stefanidis, I. (2008). Intradialytic aerobic exercise training ameliorates symptoms of restless legs syndrome and improves functional capacity in patients on hemodialysis: a pilot study. ASAIO Journal, 54(2), 185-190. doi:https://dx.doi.org/10.1097/MAT.0b013e3181641b07

Sheng, K., Zhang, P., Chen, L., Cheng, J., Wu, C., \& Chen, J. (2014). Intradialytic exercise in hemodialysis patients: a systematic review and meta-analysis. $\mathrm{Am} \mathrm{J}$ Nephrol, 40(5), 478-490. doi:10.1159/000368722

Smart, N., \& Steele, M. (2011). Exercise training in haemodialysis patients: a systematic review and meta-analysis. Nephrology (Carlton), 16(7), 626-632. doi:10.1111/j.1440-1797.2011.01471.x

Stack, A. G., Molony, D. A., Rives, T., Tyson, J., \& Murthy, B. V. (2005). Association of physical activity with mortality in the US dialysis population. American Journal of Kidney Diseases, 45(4), 690-701.

Tentori, F., Elder, S. J., Thumma, J., Pisoni, R. L., Bommer, J., Fissell, R. B., . . Robinson, B. M. (2010). Physical exercise among participants in the Dialysis Outcomes and Practice Patterns Study (DOPPS): correlates and associated outcomes. Nephrol Dial Transplant, 25(9), 3050-3062. doi:10.1093/ndt/gfq138 
Wilund, K. R., Tomayko, E. J., Wu, P. T., Ryong Chung, H., Vallurupalli, S., Lakshminarayanan, B., \& Fernhall, B. (2010). Intradialytic exercise training reduces oxidative stress and epicardial fat: a pilot study. Nephrol Dial Transplant, 25(8), 2695-2701. doi:10.1093/ndt/gfq106

Wong, J., Davis, P., Patidar, A., Zhang, Y., Vilar, E., Finkelman, M., \& Farrington, K. (2017). The Effect of Intra-Dialytic Exercise on Inflammation and Blood Endotoxin Levels. Blood Purif, 44(1), 51-59. doi:10.1159/000455059

Wiech, P., Dabrowski, M., Bazalinski, D., Salacinska, I., Korczowski, B., \& Binkowska-Bury, M. (2018). Bioelectrical Impedance Phase Angle as an Indicator of Malnutrition in Hospitalized Children with Diagnosed Inflammatory Bowel Diseases-A Case Control Study. Nutrients, 10(4). doi:10.3390/nu10040499 
SUMMARY 


\section{SUMMARY}

Sedentary lifestyle is a prevalent condition in hemodialysis (HD) patients, and is associated with an increased risk of mortality. Many factors contribute to the decreased physical activity (PA) in HD patients; these include metabolic acidosis, ureamia, inflammation and malnutrition. In addition, fatigue, lack of energy, comorbidities, lack of time and lack of access are the common reported barriers to PA by HD patients. On the other hand, active HD patients have higher quality of life (QOL). Unfortunately, there are no recommendations for PA tailored to HD patients. Another common condition and risk factor for mortality in HD patients is hyperphosphatemia. The management of hyperphosphatemia in HD patients relies on the routine dialysis, the low phosphorus $(\mathrm{P})$ diet, and the intake of phosphate binders. However, reaching the therapeutic target for $\mathrm{P}$ is not always achieved.

In an attempt to counteract sedentarism and hyperphosphatemia, and thus decrease mortality and increase quality of life (QOL), researchers are considering adjunct therapy for HD patients. The aim of this dissertation is to study the effect of intradialytic aerobic exercise among HD patients in the United Arab Emirates (UAE) on clinical outcomes, barriers to PA and QOL. The positive effect of IDE was evident in the literature; it can ameliorate solute clearance, serum P, QOL, overall health and most importantly, decrease the hospitalization rate. As in many countries, IDE programs are not yet part of the routine practice of HD patients in the UAE. In fact, this study pioneered in exploring IDE in the UAE, thus analyzing the feasibility of such a program in a developing country, since most of the evidences in this field come from developed Western countries.

In chapter 2, we conducted a systematic review on all published aerobic IDE interventions. Studies on aerobic IDE that included at least 1 of the outcome variables $[\mathrm{Kt} / \mathrm{v}$, Urea reduction ration (URR), serum P, QOL, vitamin D3, parathyroid hormone (PTH), C-reactive protein (CRP), cost effectiveness, hospitalization rate, number of emergency HD, intake of $\mathrm{P}$ binders, mortality) were included. We could not report on all of the outcome variables because some were unavailable in the selected studies. The available extracted information allowed us to conduct a meta-analysis on QOL physical component score (PCS), QOL - mental component score (MCS), dialysis efficiency $(\mathrm{Kt} / \mathrm{V})$, and P. Results of this review showed that IDE did not lead to any health hazards in the HD population, and its incorporation in the routine practice can result in significant improvement in QOL - PCS, and QOL - MCS, but not in P levels, or Kt/V. 
In Chapter 3, we presented a detailed protocol of our intervention, and the baseline sample characteristics. It was a 6 months quasi-experimental intervention on HD patients in Al Qassimi hospital in the UAE. The overall study lasted for 12 months and had 3 phases. The outcome measures were demographics and biochemical markers [Serum P mg/dl, PTH pmol/L, Calcium Phosphorus Product (Ca x P) $\mathrm{mg}^{2} / \mathrm{dl}^{2}$, Hemoglobin (HGB) $\mathrm{g} / \mathrm{dl}, \mathrm{Kt} / \mathrm{v}$, URR \%]; in addition to 4 questionnaires: 1) Malnutrition Inflammation Score (MIS) questionnaire, 2) Exercise Behavior, attitude and knowledge questionnaire, 3) Barriers to physical activity questionnaire, and 4) QOL questionnaire. In phase 1 (2 months), the research team verified the planned intervention methods and offered a 1 month individualized patient education on the importance of PA to their health. Also, the concept of IDE was introduced. At the end of this phase, we conducted the baseline assessment. In phase 2 (6 months), all consenting participants undertook the IDE training on a static pedaler, for 45 minutes, 2-3 times per week. In parallel, patients received a monthly education on the benefits of exercise for HD patients, and how to implement it safely in their lifestyle. At the end of this phase, post intervention assessment was done on all studied parameters. In phase 3 ( 4 months), the research team refrained from seeing the patients for 3 months and reassessed the patients on all parameters in the last month. Forty-one patients were included in the study, out of which $75 \%$ were hyperphosphatemic (serum $\mathrm{P}>$ $4.6 \mathrm{mg} / \mathrm{dl})$.

In Chapter 4, we explored the effects of IDE on hyperphosphatemia, MIS, URR and QOL. We found that IDE is safe and could be beneficial especially for hyperphosphatemic patients who witnessed a decrease in $\mathrm{P}$ that almost reached significance. This intervention had no significant change on URR and QOL. An interesting observation was related to the religious beliefs of the patients, which might have impeded them from objectively assigning a score to their health status on the QOL-visual analogue score; patients tended to give a high score as, otherwise, they would be defying their fate, or God's will. At the end of the study, MIS remained stable, however, because no control group was included, we could not assess whether IDE prevented the normal deterioration of the nutritional status of HD patients occurring over time.

Chapter 5 presents the effects of IDE and patient education on patients' attitudes, knowledge and barriers to PA. Also, it investigates the attitudes of nephrologists and nurses to PA in HD patients. Our results showed that the majority of the patients agreed on the importance of exercise to their health. However, the main barriers 
identified by the HD patients after the completion of the IDE program were "fatigue on dialysis days", "too many medical problems", "pain on dialysis days", and "fatigue on non-dialysis days". In addition, the provided individualized counseling reflected an increase in patients' knowledge about the safety of exercise at the end of the study. As for the nephrologists, they recognize the importance of PA in HD patients but still did not prescribe it, as they are concerned about its risks. Finally in Chapter 6, we reported a cross sectional study assessing malnutrition that we conducted on $70 \mathrm{HD}$ patients at the same hospital. It explored the concordance of the MIS and the bioelectrical impedance analyzer derived phase angle $(\mathrm{PhA})$ in the spectrum of the Global Initiative on Malnutrition (GLIM) criteria. Almost half of the sample was diagnosed as malnourished either by the MIS (48.57\%) or based on the GLIM criteria (54.29\%). Statistical analysis showed that MIS tool performed slightly better than $\mathrm{PhA}$ in the diagnosis of malnutrition in the spectrum of the GLIM criteria. In addition, knowing that ethnic differences may affect body composition, we derived the optimum cut-off point of $\mathrm{PhA}$ in our sample according to GLIM criteria, which was $\leq 5.7^{\circ}$ for males and $\leq 3.8^{\circ}$ for females.

The results of this dissertation could generate several implications for HD clinical practice in the UAE. First, it highlights the hyperphosphatemic HD patients as the target population for this type of intervention. Second, it gives an idea about the responsiveness to an IDE program in this multi-ethnic population, and the specific barriers that should be tackled to ensure patients' compliance. Also, it brings up the influence of religion on patients' perceived QOL, something that researchers should consider when evaluating QOL in this population. Finally, the above implications could serve as directions for future research in this field in the UAE. 
VALORIZATION 


\section{VALORIZATION}

Chronic kidney disease (CKD) is a global health concern (Eckardt et al., 2013) with a prevalence of $13.4 \%$ worldwide (Hill et al., 2016). In 2017, it was estimated that 3.2 million patients globally were undergoing dialysis; this number was at 3 million in 2016, thus presenting a 6\% annual growth rate (Fresenius Medical Care, 2017). In addition, $\mathrm{CKD}$ patients have increased mortality rate compared to the healthy population (Johansen et al., 2000). In 2015, the United Arab Emirates (UAE) had 1750 hemodialysis (HD) patients with an annual mortality rate of $8.5 \%$ (AlSahow et al., 2016). Furthermore, in 2018, home hemodialysis became an option for elderly, bed ridden Emirati patients (The national, 2018). All of this projects an increase in the economic burden of end stage renal disease.

Aside from the economical burden, dialysis patients present a social burden when it comes to their decreased quality of life (QOL) and their sedentary lifestyle. HD patients have decreased QOL as compared to healthy adults (Avramovic \& Stefanovic, 2012). Furthermore, QOL tends to deteriorate with the advancement in CKD stages (Aggarwal et al., 2016). The situation in the UAE is not different and chronically ill patients have lower QOL scores when compared to healthy individuals (Ayoub \& Hijjazy, 2013). Looking at HD patient's activity level, it was reported to be less than that of healthy sedentary controls (Johansen et al., 2000). As QOL and sedentarism could predict mortality rate (O'hare et al., 2003; Stack et al., 2005; Tentori et al., 2010; Avramovic \& Stefanovic, 2012), there is an urge to find an adjunctive therapy to ease the social and economical pressure of HD patients. This thesis focused on IDE as a complementary therapy for HD patients that could impact on this social and economical burden.

Today, public health awareness campaigns are highlighting the importance of exercise in a healthy lifestyle, whether you are a healthy individual or a chronically ill patient. The global attention of stakeholders and health care professionals is focused on how to improve patients' health outcomes, increase the quality of life and decrease the health care costs.

Our project supported this global perspective by being a pioneer in exploring the effect of IDE on various clinical and social outcomes in HD patients in the UAE. After the completion of our study, we could say that intradialytic aerobic exercise (IDE) could be a safe option to enhance treatment from a different angle; this could be 
possible with a minimal primary investment that covers the equipment's cost and the salary of a coordinator to assist the patients with IDE in collaboration with the nursing staff. Moreover, the initiation of IDE will require a primary assessment of the patient by his nephrologist, and the education for the staff about the benefits, and proper protocol of IDE.

Introducing IDE in HD population is a challenge, especially that many patients think of it as adding a burden to their situation. Thus, rises the need of more research focusing on the cost effectiveness of IDE, and on how it could alleviate the health and economic burden on the patient, the society, and the health care system. Cost effectiveness could be evaluated based on the hospitalization rate, the intake of phosphate binders, and maybe the frequency and duration of the weekly dialysis sessions. With the advancement of new technologies such as fitness trackers, step counter watches, and renal food applications, the motivation, assessment and accurate follow-up are now easier. In addition to the readily available, user friendly, low cost bio-impedance analyzers that offers an accurate body composition analysis. It is worth mentioning that a renal food application culturally specific for the UAE “KELA.ae" (developed by Dr. Mirey Karavetian and funded by Zayed University) has been launched in 2017. This application would not only benefit UAE dialysis patients, but also researchers in that field. It could minimize the cost of research that requires patient education on the renal diet. Such applications would also complement an IDE program, as nutrition and exercise goes together.

Besides, this dissertation highlighted the high prevalence of malnutrition among HD patients in the UAE. Therefore, future research should focus on IDE and malnutrition to position IDE as a preventive measure for the commonly reported deterioration of the nutritional status of HD patients over time (Karavetian et al., 2016).

In the UAE, $40 \%$ of the hemodialysis patients are Emirati citizens (AlSahow et al., 2016), which means that the government covers their health insurances. Hence, an initiative from the government side to support IDE programs might lessen the cost burden on the healthcare system. Also, we hope to see in the future more organizational efforts, whether from hospitals or private dialysis centers, on this matter. Organizations could take the initiative and implement IDE. For instance, a pediatric dialysis unit in Argentina is offering an amazing physical activity program for their patients. A small video about their work could be seen on https://www.youtube.com/watch?v=4mPcKXL5B70\&t=89s. "Plagiarism" in this sense would not be a crime! 


\section{REFERENCES}

Aggarwal, H. K., Jain, D., Pawar, S., \& Yadav, R. K. (2016). Health-related quality of life in different stages of chronic kidney disease. Qjm, 109(11), 711-716. doi:10.1093/qjmed/hcw054

AlSahow, A., AlRukhaimi, M., Al Wakeel, J., Al-Ghamdi, S. M., AlGhareeb, S., AlAli, F., ... Pisoni, R. L. (2016). Demographics and key clinical characteristics of hemodialysis patients from the Gulf Cooperation Council countries enrolled in the dialysis outcomes and practice patterns study phase 5 (2012-2015). Saudi J Kidney Dis Transpl, 27(6 Suppl 1), S12-23. doi:10.4103/1319-2442.194885

Avramovic, M., \& Stefanovic, V. (2012). Health-related quality of life in different stages of renal failure. Artif Organs, 36(7), 581-589. doi:10.1111/j.15251594.2011.01429.x

Ayoub, A. M., \& Hijjazi, K. H. (2013). Quality of life in dialysis patients from the United Arab Emirates. J Family Community Med, 20(2), 106-112. doi:10.4103/2230-8229.114772

Eckardt, K. U., Coresh, J., Devuyst, O., Johnson, R. J., Kottgen, A., Levey, A. S., \& Levin, A. (2013). Evolving importance of kidney disease: from subspecialty to global health burden. Lancet, 382(9887), 158-169. doi:10.1016/s01406736(13)60439-0

Fresenius Medical Care 2017. Annual Report 2017. Available from: https://www.freseniusmedicalcare.com/fileadmin/data/com/pdf/investors/New S_Publications/Annual_Reports/2017/FME_Annual_Report 2017.pdf

Hill, N. R., Fatoba, S. T., Oke, J. L., Hirst, J. A., O'Callaghan, C. A., Lasserson, D. S., \& Hobbs, F. D. (2016). Global Prevalence of Chronic Kidney Disease - A Systematic Review and Meta-Analysis. PLoS One, 11(7), e0158765. doi:10.1371/journal.pone. 0158765

Johansen, K. L., Chertow, G. M., Ng, A. V., Mulligan, K., Carey, S., Schoenfeld, P. Y., \& Kent-Braun, J. A. (2000). Physical activity levels in patients on hemodialysis and healthy sedentary controls. Kidney Int, 57(6), 2564-2570. doi:10.1046/j.1523-1755.2000.00116.x 
Karavetian, M., Elzein, H., Rizk, R., Jibai, R., \& de Vries, N. (2016). Nutritional education for management of osteodystrophy: Impact on serum phosphorus, quality of life, and malnutrition. Hemodial Int, 20(3), 432-440. doi:10.1111/hdi.12405

O'Hare, A. M., Tawney, K., Bacchetti, P., \& Johansen, K. L. (2003). Decreased survival among sedentary patients undergoing dialysis: results from the dialysis morbidity and mortality study wave 2. Am J Kidney Dis, 41(2), 447454. doi:10.1053/ajkd.2003.50055

Stack, A. G., Molony, D. A., Rives, T., Tyson, J., \& Murthy, B. V. (2005). Association of physical activity with mortality in the US dialysis population. Am J Kidney Dis, 45(4), 690-701.

Tentori, F., Elder, S. J., Thumma, J., Pisoni, R. L., Bommer, J., Fissell, R. B., . . . Robinson, B. M. (2010). Physical exercise among participants in the Dialysis Outcomes and Practice Patterns Study (DOPPS): correlates and associated outcomes. Nephrol Dial Transplant, 25(9), 3050-3062. doi:10.1093/ndt/gfq138

The National. This is no kind of life': UAE kidney patients call for dialysis at home. August 18, 2018. Available from: https://www.thenational.ae/uae/this-is-nokind-of-life-uae-kidney-patients-call-for-dialysis-at-home-1.761143 


\section{PUBLICATIONS}




\section{PUBLICATIONS}

- Salhab, N., Karavetian, M., Kooman, J., Fiaccadori, E., \& El Khoury, C. F. (2019). Effects of intradialytic aerobic exercise on hemodialysis patients: a systematic review and meta-analysis. J Nephrol. doi:10.1007/s40620-018$00565-\mathrm{z}$

- Salhab, N., Karavetian, M., Kooman, J., Fiaccadori, E. (2018). Intradialytic Aerobic Exercise in the United Arab Emirates: a Descriptive Study. Arab Journal of Nutrition and Exercise, 3(1), 18-34. doi:10.18502/ajne.v3i41.1693

- Sabatino, A., Regolisti, G., Karupaiah, T., Sahathevan, S., Sadu Singh, B. K., Khor, B. H., Salhab, N., Karavetian, M., Cupisti, A., \& Fiaccadori, E. (2017). Protein-energy wasting and nutritional supplementation in patients with endstage renal disease on hemodialysis. Clin Nutr, 36(3), 663-671. doi:10.1016/j.clnu.2016.06.007

- (ABSTRACT) Karavetian M., Salhab N., Alrukhaimi M., Kooman J., Fiaccadori E., Muthana H., Rizk R. (2018). Effect of intradialytic-aerobic exercise on clinical outcomes of hemodialysis patients in the United Arab Emirates. Clin Nutr, 37(1), S212-S213. doi:10.1016/j.clnu.2018.06.1761

- (POSTER PRESENTATION) Karavetian M., Salhab N., Kooman J., Fiaccadori E. Effect of intradialytic-aerobic exercise on clinical outcomes of hemodialysis patients in United Arab Emirates: study protocol and baseline data. 39th ESPEN Congress. The Hague, the Netherlands, 2017 
ACKNOWLEDGEMENTS 


\section{ACKNOWLEDGEMENTS}

It seemed an impossible journey, yet here I am, thanking you all...

First, allow me to express my gratitude to Maastricht University and specifically, to the School of NUTRIM for giving me the opportunity to be an external $\mathrm{PhD}$ candidate. Besides achieving a dream, this gave me the chance to visit repeatedly one of the most beautiful cities I have ever seen, Maastricht!

Next, my deepest appreciation goes to my supervisor, Dr. Jeroen Kooman. Thank you for your availability, always finding the time in your busy schedule to skype, meet, or send me detailed responses and feedbacks on my work. I appreciate your valuable advices and your guidance. You encouraged and supported me to attend educational courses to improve my knowledge and skills. You ensured I get the best treatment when I got a small, second degree burn at UM.

To my co-supervisor, Dr. Enrico Fiaccadori, thank you for your guidance and trust. You helped me grow my network and connect with fellow researchers. I sincerely thank you for your valuable time and effort you had put to review my work.

To my friend, my daily co-supervisor, Dr. Mirey Karavetian, my gratefulness to you goes beyond the words of thank you. I was lucky that I met you in 2006, and I never thought that you would be the one who will put me on the track that I always wanted to take. You trusted my abilities and you believed in me. I could not have done this without your continuous guidance, counseling, advises and follow ups. You helped me open doors that I thought I could never knock. I learned a lot from you, I learned how to become a researcher. With your positive energy, you made every step of this journey look easy. This will change my life, and I owe you this accomplishment.

Many thanks to everyone who contributed to this thesis, namely Dr. Khaled Abesh, Dr. Rana Rizk, Mrs. Cosette Fakih, and Mrs. Aida Farha. I am very thankful to the research assistants that helped me, and to staff and patients in the dialysis unit for their cooperation.

To my family, my mom, my sister and my brother, for all your support and encouragement; for taking care of my kids when I am away as if they where yours. To my mother in law, my sisters in law Rita and Dorine, for all your support. To my 
friend, Marielle, thank you for helping me out when I could not reach to pick up my boys from school or football.

To my boys, Christopher and Ralph, I love you. You gave up a lot of your "mommy" time for me to fulfill my PhD. You were the reason I never gave up. I hope that this will teach you that you can achieve anything you want in your life if you decide to.

Last, to my loving and supporting husband. You praised every small step I took along the way, and this meant a lot for me. You gave me confidence when I needed it. Thank you for being my partner for the last 24 years and forever.

Finally, I dedicate this thesis to the souls of my father and father in law, who were true examples of unconditional giving and love.

\section{Nada}


CURRICULUM VITAE 


\section{CURRICULUM VITAE}

Nada Salhab was born on April 29 ${ }^{\text {th }}, 1979$, in Lebanon. She earned her bachelor degree in "Nutrition and Dietetics" from the American University of Beirut, Lebanon, in 2001, and her masters of science in "Nutrition" from Texas Woman's University, United States, in 2003. She completed her dietetics internship from Hotel Dieu de France Hospital, Lebanon, in 2004. She is currently a PhD candidate in the school of "Nutrition and Translational Research in Metabolism" in Maastricht University, Netherlands. She conducted her research at Al Qassimi Hospital in the United Arab Emirates (UAE).

Mrs. Salhab is a licensed nutritionist and dietitian by the Ministry of Public Health in Lebanon since 2006. She also holds a professional license from Dubai Health Authority in the UAE since 2009. While completing her masters degree, she earned a teaching assistantship and worked as an instructor at the biology department for 2 years. Nada has been working as a clinical dietitian (mainly in polyclinics) since 2004 in Saudi Arabia, Lebanon, and the UAE consecutively. While in Lebanon in 2006, she also worked as a research assistant at the faculty of Nutrition and Dietetics at the American University of Beirut. 
